\title{
Characterization of Diamond Sensors for use in ATLAS Calorimetry Upgrades
}

\author{
by \\ Joshua Turner
}

\author{
A thesis submitted to the \\ Faculty of Graduate and Postdoctoral Affairs \\ in partial fulfillment of the requirements \\ for the degree of \\ Master of Science \\ Department of Physics \\ Carleton University \\ Ottawa-Carleton Institute for Physics \\ Ottawa, Canada \\ May 16, 2012
}

Copyright (C) 2012 Joshua Turner 
Library and Archives

Canada

Published Heritage

Branch

395 Wellington Street

Ottawa ON K1A ON4

Canada
Bibliothèque et

Archives Canada

Direction du

Patrimoine de l'édition

395 , rue Wellington

Ottawa ON K1A ON4

Canada
Your file Votre référence

ISBN: 978-0-494-91508-0

Our file Notre référence

ISBN: $978-0-494-91508-0$
NOTICE:

The author has granted a nonexclusive license allowing Library and Archives Canada to reproduce, publish, archive, preserve, conserve, communicate to the public by telecommunication or on the Internet, loan, distrbute and sell theses worldwide, for commercial or noncommercial purposes, in microform, paper, electronic and/or any other formats.

The author retains copyright ownership and moral rights in this thesis. Neither the thesis nor substantial extracts from it may be printed or otherwise reproduced without the author's permission.
AVIS:

L'auteur a accordé une licence non exclusive permettant à la Bibliothèque et Archives Canada de reproduire, publier, archiver, sauvegarder, conserver, transmettre au public par télécommunication ou par l'Internet, prêter, distribuer et vendre des thèses partout dans le monde, à des fins commerciales ou autres, sur support microforme, papier, électronique et/ou autres formats.

L'auteur conserve la propriété du droit d'auteur et des droits moraux qui protege cette thèse. $\mathrm{Ni}$ la thèse ni des extraits substantiels de celle-ci ne doivent être imprimés ou autrement reproduits sans son autorisation.
In compliance with the Canadian Privacy Act some supporting forms may have been removed from this thesis.

While these forms may be included in the document page count, their removal does not represent any loss of content from the thesis.
Conformément à la loi canadienne sur la protection de la vie privée, quelques formulaires secondaires ont été enlevés de cette thèse.

Bien que ces formulaires aient inclus dans la pagination, il n'y aura aucun contenu manquant. 


\section{Abstract}

The construction of forward calorimeters in high-rate environments, such as those found in the ATLAS detector, requires the use of radiation hard materials. The luminosity upgrade proposed for the Large Hadron Collider (LHC) will result in an instantaneous particle flux that will result in some significant signal degradation in the current Forward Calorimeter (FCal). The installation of a small calorimeter, known as the Mini-FCal, between the FCal and the ATLAS interaction point has been proposed to ensure the continued operation of the FCal. Polycrystalline Chemical Vapour Deposited (pCVD) diamond is one of the active materials being explored for the Mini-FCal. Four such diamonds have been irradiated to the integrated flux levels expected in the Mini-FCal $\left(5 \times 10^{17}\right.$ protons $\left./ \mathrm{cm}^{2}\right)$ and have remained operational, although at $5 \%$ of their original signal amplitude. The variations in signal response over the surface of a single pCVD diamond detector have been examined, with the goal of determining their suitability for use in the Mini-FCal. A single particle was measured to deposit a mean charge of 5.05 ke with a standard deviation of $0.70 \mathrm{ke}$. 
This thesis is dedicated to Serena, Connor, and Keely, who alternated between keeping me sane and driving me crazy throughout its creation. 


\section{Acknowledgements}

I would like to acknowledge the following people for their support during the course of my studies:

To my supervisor Gerald Oakham, who provided me with the opportunity to continue this research. Thanks go out to Yun-Ha Shin, Dave Axen, Adam Robichaud, Philippe Gravelle, and Yves Baribeau, without whom this research likely would not have been completed.

Thank you to Koloina Randrianarivony and Daniel Duque for their aid organizing and assembling the spatial test, and to all the shifters who took the time to help us: Leonid Kurchaninov, Peter Krieger, Claude Leroy, and Frederick Dallaire.

I would like to thank my dogs Connor and Keely for keeping me company during the long hours spent writing this thesis, and for not destroying anything important.

Finally, to Serena: thanks for making this bearable. You made this possible in more ways than I can count. 


\section{Statement of Originality}

The analysis of the irradiation of pCVD diamond detectors that occurred at TRIUMF in September of 2010 (Chapter 3) was originally published in Volume 6 of the Journal of Instrumentation in May of 2011 under the title "Diamond detector irradiation tests at TRIUMF". I took part in the test as a shifter, monitoring the DAQ system over the course of 12 days.

I had a significant role in the spatial uniformity test (Chapters 4 and 5). I ensured that the equipment used in the test operated correctly, that the equipment was shipped to and from TRIUMF, and that the equipment was in place in the beam area and operational in time for the test and for the duration of the test. The analysis of the spatial uniformity data was entirely my work. 
Table 1: Table of Acronyms.

\begin{tabular}{|l|l|}
\hline Acronym & Meaning \\
\hline ALICE & A Large Ion Collider Experiment \\
BCID & Bunch Crossing IDentifier \\
ccd & Charge Collection Distance \\
CERN & European Organization for Nuclear Research \\
CMS & Compact Muon Solenoid \\
DAQ & Data AcQuisition \\
e-h & electron-hole \\
EM & ElectroMagnetic \\
EMEC & ElectroMagnetic End-Cap \\
FADC & Flash Analog-to-Digital Converter \\
FCal & Forward Calorimeter \\
FE-I3 & Front End-Iteration 3 \\
FEA & Finite Element Analysis \\
FPGA & Field Programmable Gate Array \\
GPIB & General Purpose Interface Bus \\
HEC & Hadronic End-Cap \\
HL-LHC & High Luminosity Large Hadron Collider \\
HV & High Voltage \\
IBL & Insertable B-Layer \\
\hline
\end{tabular}




\begin{tabular}{|l|l|}
\hline Acronym & Meaning \\
\hline ID & Inner Detector \\
LHC & Interaction Point \\
LHCb & Large Hadron Collider \\
MIP & Large Hadron Collider beauty \\
NIEL & Nonimum Ionizing Particle \\
NIM & Nuclear Instrumentation Module \\
p/n & positive to negative junction \\
pCVD & polycrystalline Chemical Vapour Deposited \\
R\&D & Research and Development \\
S/N & Signal to Noise ratio \\
scCVD & single crystal Chemical Vapour Deposited \\
SCT & SiliCon Tracker \\
sFCal & super Forward Calorimeter \\
TDC & Time-to-Digital Converter \\
ToF & Time of Flight \\
ToT & Time over Threshold \\
TRT & Transition Radiation Tracker \\
VME & Versa Module European \\
VNC & Virtual Network Computing \\
USB & Universal Serial Bus \\
\hline
\end{tabular}




\section{Contents}

1 Introduction 1

1.1 Large Hadron Collider . . . . . . . . . . . . . . . . . 2

1.2 A Toroidal LHC Apparatus $\ldots \ldots \ldots \ldots$

1.2.1 Inner Detector $\ldots \ldots \ldots \ldots \ldots \ldots \ldots$

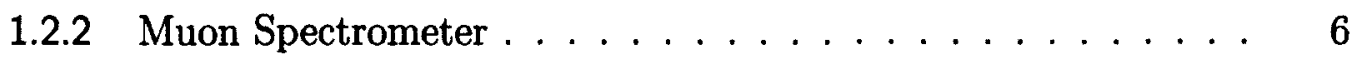

1.2 .3 Calorimetry $\ldots \ldots \ldots \ldots \ldots \ldots \ldots \ldots \ldots \ldots$

1.3 Forward Calorimeter $\ldots \ldots \ldots \ldots \ldots \ldots$

1.3.1 Design $\ldots \ldots \ldots \ldots \ldots \ldots \ldots \ldots \ldots \ldots$

1.4 High Luminosity Large Hadron Collider $\ldots \ldots \ldots \ldots$

1.4.1 Forward Calorimetry at the HL-LHC $\ldots \ldots \ldots \ldots$

1.4.11. Super FCal . . . . . . . . . . . . . . . 14

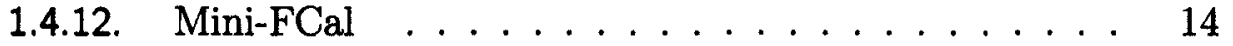

2 Fundamentals of Calorimetry and Semiconductor Detectors 18

2.1 Particle Interactions in Matter $\ldots \ldots \ldots \ldots \ldots \ldots$ 
2.1.1 Charged Particle Interactions $\ldots \ldots \ldots \ldots \ldots$

2.1.2 Neutral Particle Interactions $\ldots \ldots \ldots \ldots . \ldots \ldots 22$

2.1 .3 Photonic Interactions $\ldots \ldots \ldots \ldots \ldots \ldots$

2.2 Electromagnetic Cascades $\ldots \ldots \ldots \ldots \ldots \ldots$

2.3 Hadronic Cascades . . . . . . . . . . . . . . . . . . 23

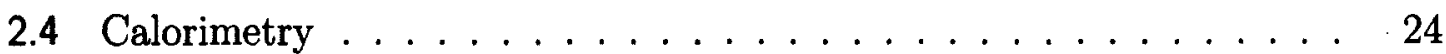

2.4.1 Multiple Scattering $\ldots \ldots \ldots \ldots \ldots \ldots$

2.5 Properties of Semiconducting Detectors $\ldots \ldots \ldots \ldots \ldots$

2.5.1 The Shockley-Ramo Theorem . . . . . . . . . . . 27

2.5.2 Properties of Silicon Detectors $\ldots \ldots \ldots \ldots \ldots$

2.5.3 Properties of Diamond Detectors $\ldots \ldots \ldots \ldots \ldots$

2.5.4 Operation of a Diamond Detector $\ldots \ldots \ldots \ldots \ldots$

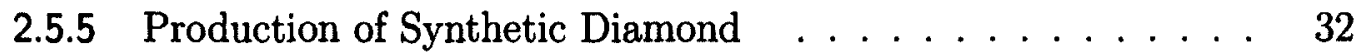

2.6 Radiation Damage $\ldots \ldots \ldots \ldots \ldots \ldots \ldots$

2.6.1 Effect of Radiation Damage in Semiconductor Detectors _.. 35

3 Irradiation of pCVD Diamond Detectors 38

3.1 Introduction $\ldots \ldots \ldots \ldots \ldots \ldots \ldots \ldots \ldots \ldots \ldots$

3.2 Results . . . . . . . . . . . . . . . . . . . . 41

3.3 Summary of Irradiation Test $\ldots \ldots \ldots \ldots \ldots \ldots \ldots$ 
4 Characterization of pCVD Diamond Detectors in Preparation for Spatial Uniformity Measurements

4.1 Diamond Detector Properties $\ldots \ldots \ldots \ldots \ldots \ldots$

4.1 .1 Laboratory Tests . . . . . . . . . . . . . . . . . 46

4.2 Detector for Spatial Measurements . . . . . . . . . . . 53

4.2.1 FE-13 Readout Electronics $\ldots \ldots \ldots \ldots \ldots \ldots$

$4.2 .2 \quad$ USBpix $\ldots \ldots \ldots \ldots \ldots \ldots \ldots \ldots \ldots$

4.3 Data Acquisition System $\ldots \ldots \ldots \ldots \ldots$

4.4 Diamond Spatial Uniformity Test $\ldots \ldots \ldots \ldots \ldots$

5 pCVD Diamond Spatial Uniformity Measurements 64

5.1 USBpix and Scintillator Analysis $\ldots \ldots \ldots \ldots \ldots \ldots$

5.2 Diamond Analysis $\ldots \ldots \ldots \ldots \ldots \ldots \ldots$

5.2.1 Calibration of Diamond Detector Signal _ . . . . . . . 75

5.2 .2 Data Quality . . . . . . . . . . . . . . . . 82

5.3 Spatial Response . . . . . . . . . . . . . . 85

5.3.1 Signal Amplitude Variations with Bias Voltage Polarity _. . 88

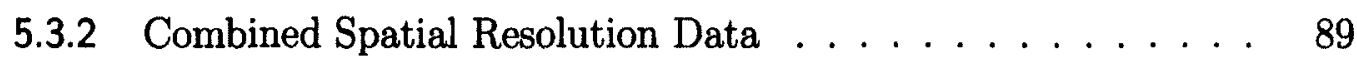

6 Summary and Outlook 100

$\begin{array}{ll}\text { References } & 102\end{array}$ 


\section{List of Tables}

1 Table of Acronyms. . . . . . . . . . . . . vi

2.1 Definition of variables used in the Bethe-Bloch equation . . . . . 20

2.2 Material properties of silicon and diamond. . . . . . . . 30

3.1 Properties of diamond detectors used in the irradiation test at TRIUMF. 39

4.1 Properties of diamond detectors available for use in spatial uniformity

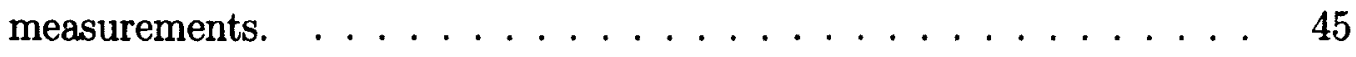

4.2 Scintillator dimensions. . . . . . . . . . . . . . . . . . . 61

5.1 Parameters for each data set gathered during spatial uniformity test. $\quad 66$

5.2 Fit parameters for each signal amplitude measurement method and the amplitude of the attenuated output of the pulse generator. . . . . 78

5.3 Average signal amplitude and statistical uncertainty from three different measurement methods over the course of data set $2 \ldots \ldots \ldots 8$

5.4 Signal amplitude mean and standard deviation for all data sets. . . . 90 
5.5 Mean and standard deviation of the three combined amplitude maps and the data sets used to create them. . . . . . . . . . . . 99 


\section{List of Figures}

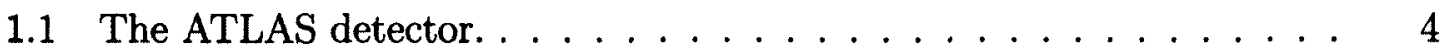

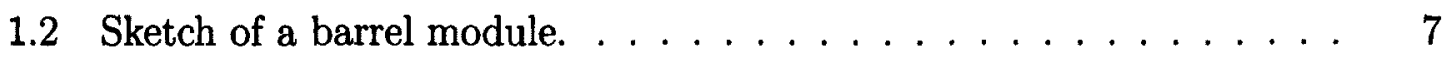

1.3 An end-cap cryostat. $\ldots \ldots \ldots \ldots \ldots \ldots$

1.4 An FCal module. . . . . . . . . . . . . . . . . . 10

1.5 An FCall active gap. . . . . . . . . . . . . . . . . 11

1.6 The Mini-FCal. . . . . . . . . . . . . . . . . . . 15

2.1 Depiction of charge collection in a CVD diamond detector. . . . . . 31

2.2 Summary of proton irradiation results for CVD diamond. . . . . . . 36

3.1 Response of three diamond detectors at the beginning of the test. . . 40

3.2 Response of the four detectors as a function of fluence. . . . . . . 42

4.1 Cremat amplifier used for spatial uniformity measurements. . . . . 47

4.2 Response of diamond detector B after exposure to UV radiation. . . . 48

4.3 Leakage current produced by diamond detector B. . . . . . . . . . 50 
4.4 Leakage current produced by diamond detector $1 . \ldots . . . . . . .50$

4.5 Leakage current produced by diamond detector $2 . \ldots . . . . . .51$

4.6 Leakage current produced by diamond detector $6 . \ldots 51$

4.7 Hysteresis data for diamond detector B. . . . . . . . . . . . . 52

4.8 USBpix readout system. . . . . . . . . . . . . . . 54

4.9 Circuit diagram for spatial uniformity test. . . . . . . . . . . 56

4.10 Custom holder designed for use in spatial uniformity test. . . . . . . 58

4.11 Diagram of components exposed to particle beam during spatial uniformity test. . . . . . . . . . . . . . . . . 59

4.12 Placement of scintillators in M11 beam area. . . . . . . . . . 60

4.13 Placement of detectors in M11 beam area. . . . . . . . . . . . . 60

5.1 Orientation of diamond detector pre and post rotation with respect to the USBpix. .............................. 65

5.2 Diagram of possible events in the USBpix silicon sensor. . . . . . 67

5.3 Unmasked pixel occupancy for the USBpix detector for data set 2. . 69

5.4 Masked pixel occupancy for the USBpix detector for data set 2. . . 69

5.5 ToT data produced by the USBpix for data set $2 . \ldots \ldots$. . . . 70

5.6 Histogram of the time difference measured between scintillators 0 and

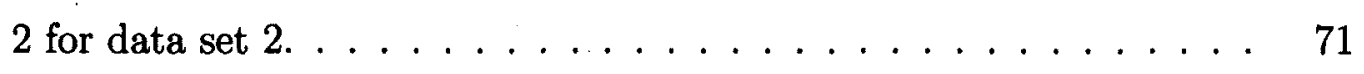

5.7 Sample waveforms from the diamond detector. . . . . . . . . . 72 
5.8 Averaged waveforms from the diamond detector. . . . . . . . . 72

5.9 Location of pixels used to measure electronic background in data set 2. 73

5.10 Averaged signal background. . . . . . . . . . . . . . 73

5.11 Averaged signal waveform after the removal of signal background. . . 74

5.12 Signal integral range tests. . . . . . . . . . . . . 74

5.13 Averaged signal from a pixel in data set 2 fit to the averaged signal from data set $2 \ldots \ldots \ldots \ldots \ldots \ldots \ldots \ldots$

5.14 Average of 2000 waveforms produced by the Cremat amplifier for two

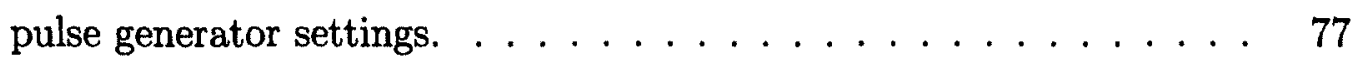

5.15 Signal integral calibration data. . . . . . . . . . . 79

5.16 Peak amplitude calibration data. $\ldots \ldots \ldots \ldots \ldots \ldots$

5.17 Fit amplitude calibration data. $\ldots \ldots \ldots \ldots$. . . . . 80

5.18 Output from pulse generator measured by the oscilloscope after attenuation. . . . . . . . . . . . . . . . . 80

5.19 Measurement of average peak amplitude per scan for data set 1 . . . 83

5.20 Measurement of average peak amplitude per scan for data set $5 \ldots$. . 83

5.21 Variation in peak timing during data set $1 \ldots \ldots \ldots . \ldots 84$

5.22 Variation in peak timing during data set $4 \ldots \ldots \ldots \ldots$

5.23 Variations in pedestal value in data set $2 \ldots \ldots \ldots \ldots$

5.24 Variations in the signal amplitude measured per scan in data set $4 . \quad 86$

5.25 Averaged amplitude map for data set $2 \ldots \ldots \ldots \ldots$ 
5.26 Comparison of diamond signal amplitude to number of hits in USBpix

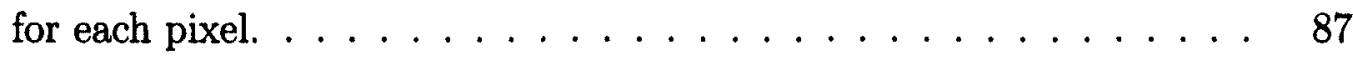

5.27 Diamond detector amplitude maps. . . . . . . . . . . . . . 89

5.28 Amplitude spectra for data sets 3 and $4 \ldots \ldots \ldots \ldots \ldots$

5.29 Amplitude maps from data sets 2 (left) and 3 (right) . . . . . . 91

5.30 Map of the sum of the 10 highest amplitude combined pixels for each 50 micron shift. . . . . . . . . . . . . . . . . . 93

5.31 Map of the sum of the 100 highest amplitude combined pixels for each

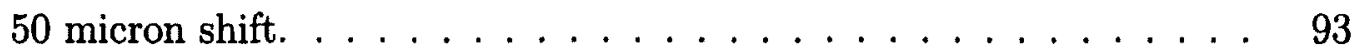

5.32 Map of the sum of the 1000 highest amplitude combined pixels for each 50 micron shift. . . . . . . . . . . . . . . . . . . . . 94

5.33 Combined amplitude map for data sets 2 and 3 in 50 micron $\times 50$

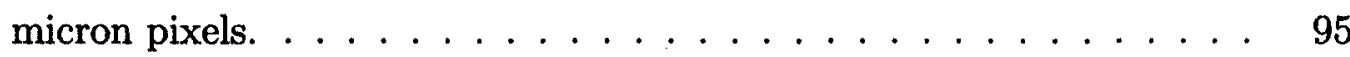

5.34 Combined amplitude map for data sets 2 and 3 in 100 micron $\times 100$

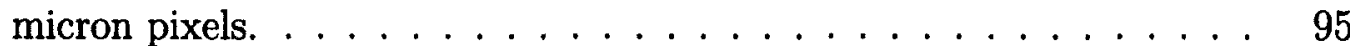

5.35 Combined amplitude map for data sets 2 and 3 in 200 micron $\times 200$

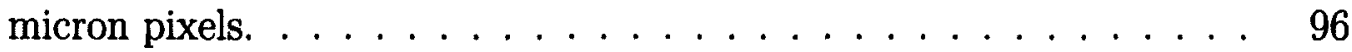

5.36 Amplitude spectrum for 50 micron $\times 50$ micron pixels. . . . . . . 96

5.37 Amplitude spectrum for 100 micron $x 100$ micron pixels. . . . . . . 97

5.38 Amplitude spectrum for 200 micron $\times 200$ micron pixels. . . . . . . 97

5.39 Amplitude spectrum for data set $2 \ldots \ldots \ldots \ldots \ldots \ldots$

5.40 Amplitude spectrum for data set $3 \ldots \ldots \ldots \ldots \ldots$ 


\section{Chapter 1}

\section{Introduction}

Particle physics today is embodied by the Standard Model of elementary particles [1]. It theorizes that matter as we know it consists of fundamental spin- $-\frac{1}{2}$ particles known as fermions and integer spin particles known as bosons, which mediate fermion interactions. This theory describes the electromagnetic, strong and weak interactions, but does not include gravitational interactions.

In an attempt to explain the origin of mass, Philip Warren Andersen [2] proposed a method by which mass could be imparted to particles, which was further developed by Peter Higgs [3], Robert Brout and Francois Englert [4], and Gerald Guralnik, C. R. Hagen, and Tom Kibble [5]. These theories result in the prediction of a spin-0 boson, which has since become known as the Higgs boson. It remains the only unobserved particle predicted by the Standard Model. Several colliders and detectors, most notably the Large Hadron Collider (LHC) and its four experiments have been designed largely to either confirm or deny the existence of the Higgs boson. 


\subsection{Large Hadron Collider}

The LHC occupies a circular $27 \mathrm{~km}$ tunnel $100 \mathrm{~m}$ beneath the Franco-Swiss border near Geneva, Switzerland, at the European Organization for Nuclear Research (CERN). It accelerates particles (either protons or ions, depending on the operating phase) through two rings traveling in opposite directions. The accelerator complex makes use of the existing PS and SPS machines to gradually accelerate particles prior to injection into the LHC ring. At the nominal operating point, the LHC will accelerate 2808 bunches of $1.1 \times 10^{11}$ protons to $7 \mathrm{TeV}$, or $0.999999991 \mathrm{c}$, per ring, at a bunch spacing of $25 \mathrm{~ns}$ and an instantaneous luminosity of $10^{34} \mathrm{~cm}^{-2} \mathrm{~s}^{-1}$.

The accelerator uses approximately 6000 superconducting dipole and quadrupole magnets to bend and focus the particle beams respectively, as well as over 3000 other superconducting and conducting magnets. The superconducting magnets are cooled to $1.9 \mathrm{~K}$ using 120 tonnes of liquid helium. To increase the proton beam energy up to the nominal $7 \mathrm{TeV}$, the magnets must be capable of producing magnetic fields that vary in strength from $0.54 \mathrm{~T}$ to $8.3 \mathrm{~T}$ (for comparison, the earth's magnetic field is measured in tens of $\mu \mathrm{T}$ ). The two beams of particles are kept separate while circulating around most of the ring. They collide only in four places; the interaction points of the four detectors.

The LHC houses four major experiments, which are designed to explore various frontier physics. The CMS [6] and ATLAS [7] experiments are general purpose detectors designed to search for the Higgs boson and to explore a wide range of highenergy physics phenomena. The $\mathrm{LHCb}[8]$ is designed specifically to examine physics involving the b-quark and the matter-antimatter asymmetry currently seen in the observable universe. The ALICE [9] detector is designed to examine lead-ion collisions 
at energies of $2.8 \mathrm{TeV}$ per nucleon, creating a quark-gluon plasma, a state of matter that existed in the early stages of the universe. A first look at $\mathrm{Pb}-\mathrm{Pb}$ ion collisions in ATLAS [10] showed the first observation of the phenomenon known as jet quenching within a quark-gluon plasma and provided the LHC's first new physics.

The LHC briefly became operational in September of 2008 before an unfortunate failure [11] lead to damage to the beam line. Repairs took until November 2009, when the LHC was brought back online but was limited to a lower energy at that time to ensure safe running. In 2011 the LHC was producing collisions with a centre of mass energy of $7 \mathrm{TeV}$ at an instantaneous luminosity of up to $3.5 \times 10^{33}$

$\mathrm{cm}^{-2} \mathrm{~s}^{-1}$, and is currently expected to reach an energy of $8 \mathrm{TeV}$ and close to its design luminosity in 2012. Further work will be done during the 2013-2014 LHC shutdown to allow operation at the design energy, with the possibility of exceeding the design luminosity. For a comprehensive review of the LHC, see [12].

\subsection{A Toroidal LHC Apparatus}

A Toroidal LHC ApparatuS, or ATLAS, is one of the two general purpose experiments installed at the LHC, and is designed to observe a wide range of possible phenomena expected in the $\mathrm{TeV}$ energy range. Over 3000 physicists from 38 countries are members of the ATLAS collaboration, including over 1000 students. A complete discussion of the ATLAS detector ean be found in [7].

The design of the ATLAS detector was motivated by the search for the Higgs boson and some of the more promising theories of physics beyond the standard model. The ATLAS detector consists of three major detector subsystems: the Inner Detector (ID), nearest the beamline, measures particle sign, position, and momentum. The 


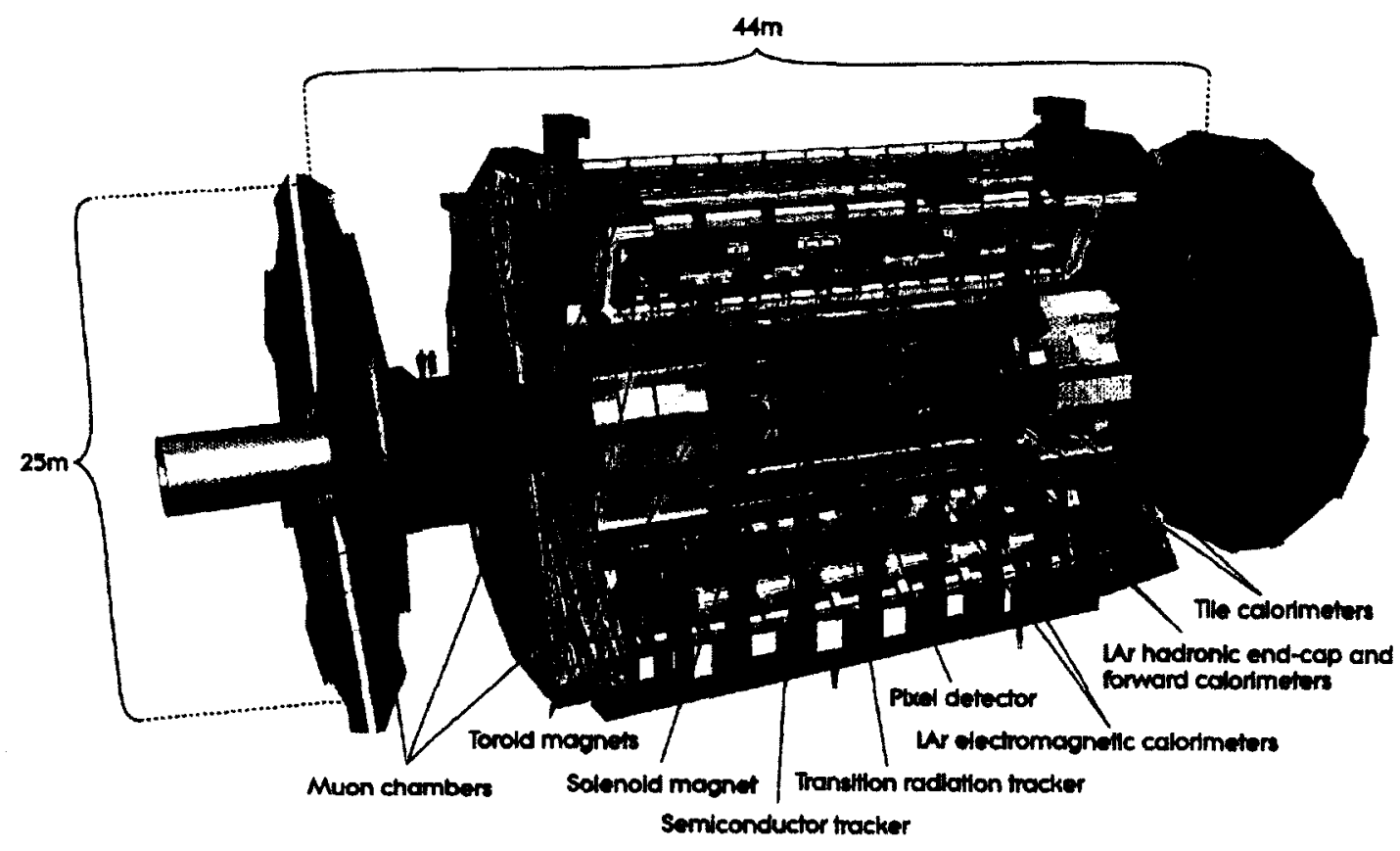

Figure 1.1: The ATLAS detector. [7] 
ID is surrounded by the calorimeter system, which is used to reconstruct particle energy. The Muon Spectrometer, the detector subsystem furthest from the beamline, is designed to detect and measure the momentum of particles that penetrate the calorimeters. Muons are the only charged particles capable of reaching the Muon Spectrometer from the interaction point (IP) of the ATLAS detector, hence the name. An illustration of the ATLAS detector is given in Figure 1.1.

\subsubsection{Inner Detector}

The ID is the innermost region of the ATLAS detector and extends approximately $1 \mathrm{~m}$ radially from the beam pipe. It consists of three distinct subsystems: a set of pixel detectors consisting of three cylindrical layers in the barrel region and three discs in the end-cap regions, extending from $\mathrm{R}=50.5 \mathrm{~mm}$ to $149.6 \mathrm{~mm}$, the Silicon micro-strip Tracker (SCT) consisting of four cylindrical layers in the barrel region and nine discs in the end-cap regions extending from $\mathrm{R}=275 \mathrm{~mm}$ to $560 \mathrm{~mm}$, and the Transition Radiation Tracker (TRT) extending from $R=563 \mathrm{~mm}$ to $1066 \mathrm{~mm}$. The entire system is immersed within a $2 \mathrm{~T}$ solenoidal magnetic field, and is designed to resolve the approximately 1000 particle tracks created at the interaction point every $25 \mathrm{~ns}$ at the LHC's design luminosity.

The ID is designed to measure the origin, trajectory, momentum and sign of charged particles created within the ATLAS detector. On average, a particle traverses three pixel detectors, eight strip layers in the SCT, and 36 straw layers in the TRT, from which the particle trajectory can be reconstructed. The momentum and relative charge of particles can be reconstructed from the curvature of the observed trajectories. For an in-depth discussion of the ID, see references [13][14]. 


\subsubsection{Muon Spectrometer}

The Muon Spectrometer forms the outermost region of the ATLAS detector. It is designed to detect charged particles exiting the calorimeters and to measure their momentum in the pseudorapidity ${ }^{1}$ range $|\eta|<2.7$ while triggering on these particles in the region $|\eta|<2.4$. For a more detailed treatment of the Muon Spectrometer, see reference [15].

\subsubsection{Calorimetry}

Calorimeters are used to reconstruct the energy of particles that interact with them. This is accomplished in ATLAS by sampling the energy deposited and the shape of the cascades produced by the particles. Calorimeters are often divided into two sub-groups: electromagnetic (EM) calorimeters, which contain particles that interact mainly via the EM process, such as electrons and photons, and hadronic calorimeters, which add sufficient material to contain particles that interact mainly through nuclear collisions and produce more penetrating particle cascades.

The calorimeters of the ATLAS detector are divided into three regions. The entire ATLAS calorimeter system is hermetic, providing full coverage in $\phi$ and for $|\eta|<4$.9. The barrel region calorimetry system consists of the Electromagnetic Barrel Calorimeter, a lead-liquid argon sampling calorimeter with an accordion geometry, shown in Figure 1.2, providing full coverage in $\phi$ and for $|\eta|<1.475$, and the Tile Calorimeter, a sampling calorimeter that uses steel as its absorber and scintillator as

\footnotetext{
${ }^{1}$ ATLAS uses a right-handed coordinate system with its origin at the nominal interaction point (IP) in the centre of the detector and the z-axis along the beam pipe. The pseudorapidity is defined in terms of the polar angle, $\theta$, as $\eta=-\ln \tan (\theta / 2)$.
} 


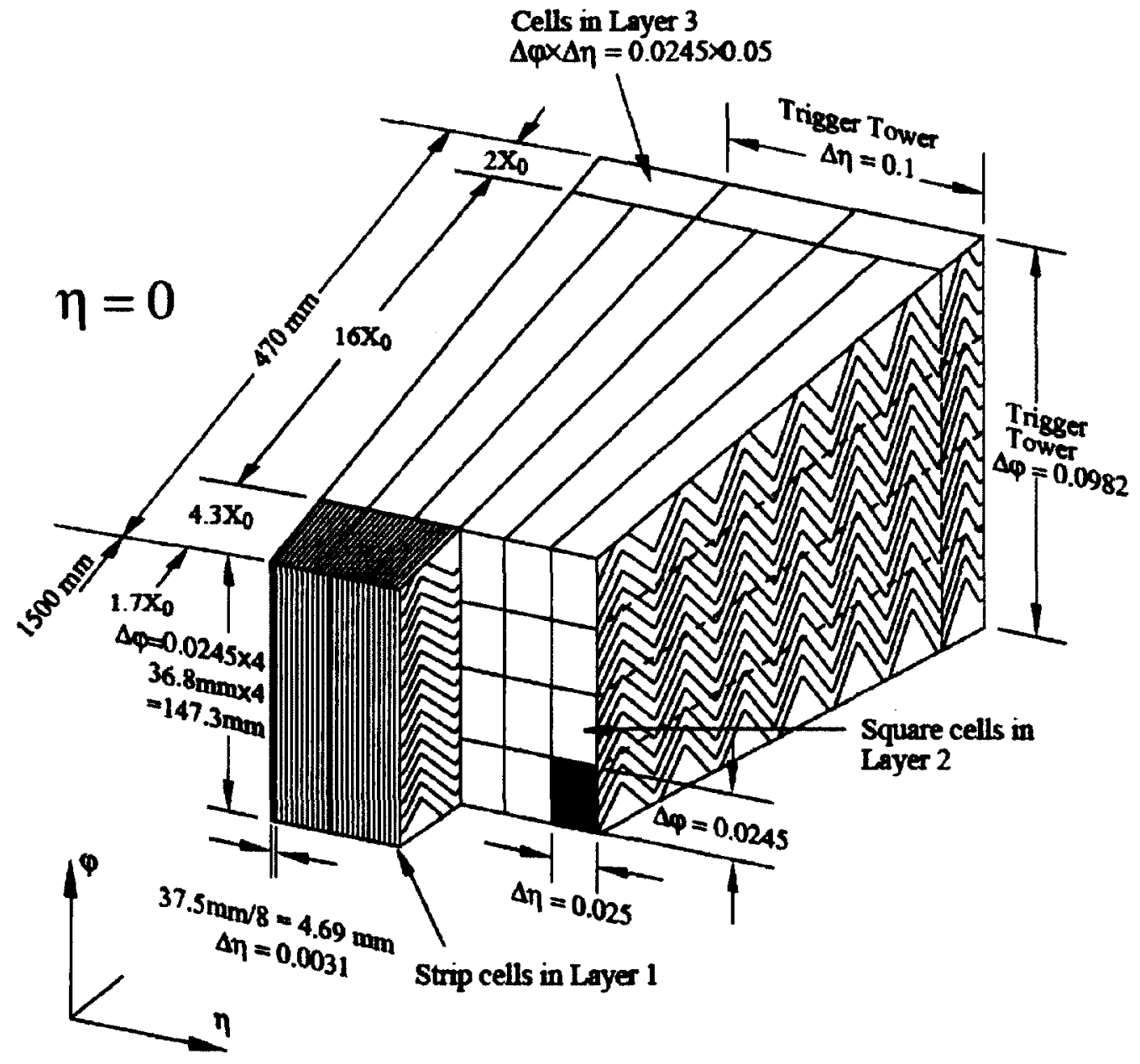

Figure 1.2: Sketch of a barrel module. Due to the overlapping of the accordion structure between modules, the barrel region provides complete coverage in $\phi$. [7] 
its active material, which is located behind the Electromagnetic Barrel Calorimeter in the region $|\eta|<1.7$.

The two end-cap calorimeter systems each consist of an Electromagnetic EndCap Calorimeter (EMEC), a Hadronic End-Cap Calorimeter (HEC), and a Forward Calorimeter (FCal), all of which use liquid argon as their sampling material. Each EMEC covers the region $1.375<|\eta|<3.2$ and consists of two co-axial wheels. The gap between the two wheels is $3 \mathrm{~mm}$ and is located at $|\eta|=2.5$. Each wheel is subdivided into eight accordion wedges, the design of which prevents any discontinuities in azimuthal coverage. Each HEC is a copper-liquid argon sampling calorimeter with a standard parallel-plate design, covering the range $1.5<|\eta|<3.2$. The HEC calorimeters consist of two wheels, the inner wheel (HEC1), and the outer wheel (HEC2). Each wheel consists of 32 wedge-shaped modules. Finally, the FCal modules cover the region from $3.1<|\eta|<4.9$.

\subsection{Forward Calorimeter}

The FCals are located approximately $4.7 \mathrm{~m}$ from the interaction point of the ATLAS experiment in the end-cap cryostats between the beam pipe and the HEC modules, as shown in Figure 1.3, and enclose as much of the forward region as is reasonable.

The FCal's main purpose is to provide jet energy measurements and to improve missing $E_{T}$ measurements. Weakly interacting particles will escape detection in the ATLAS experiment. However, their presence can be inferred in events with large momentum/energy imbalance in the direction transverse to the beam. The technique of summing energy vectors to look for an energy imbalance is known as a missing $E_{T}$ measurement. Poorly measured jets or jets that escape detection create the equivalent 


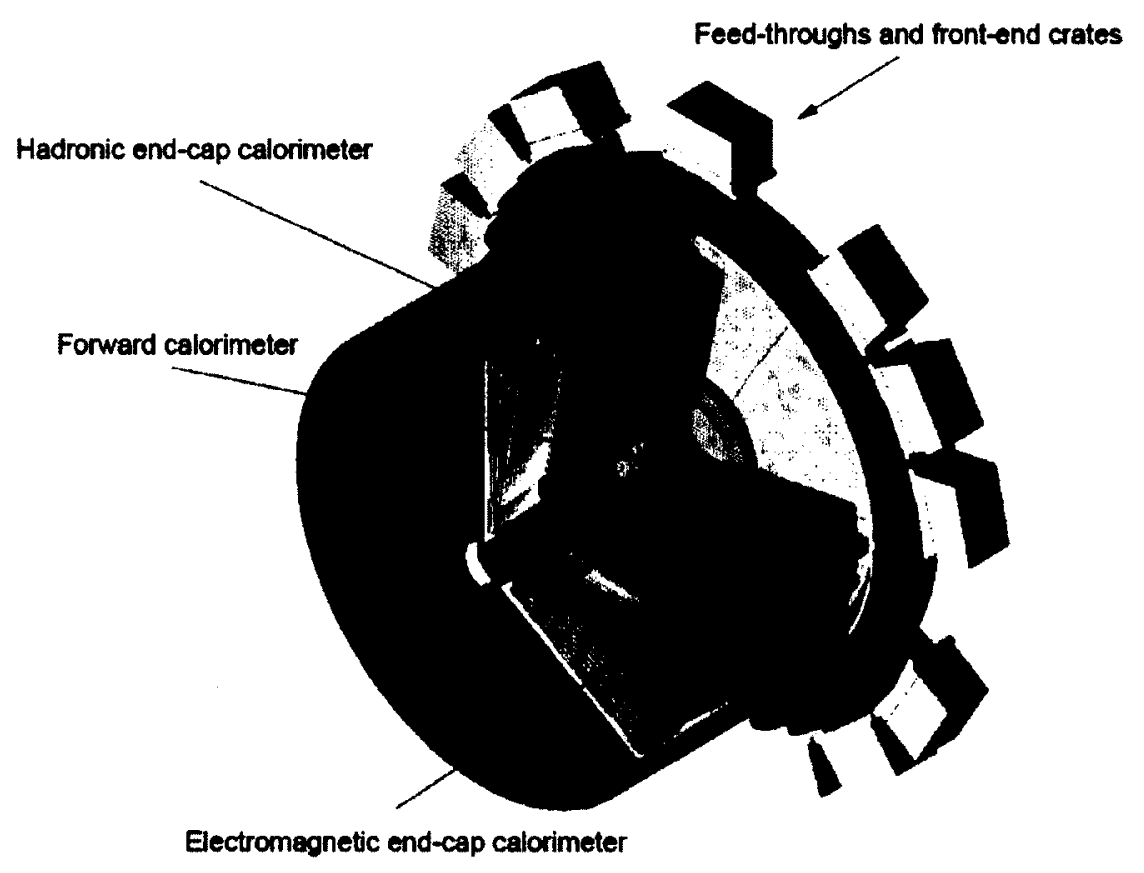

Figure 1.3: An end-cap cryostat. The interaction point is towards the bottom left. The three end-cap calorimeters and the front-end crates are labeled. [7] 


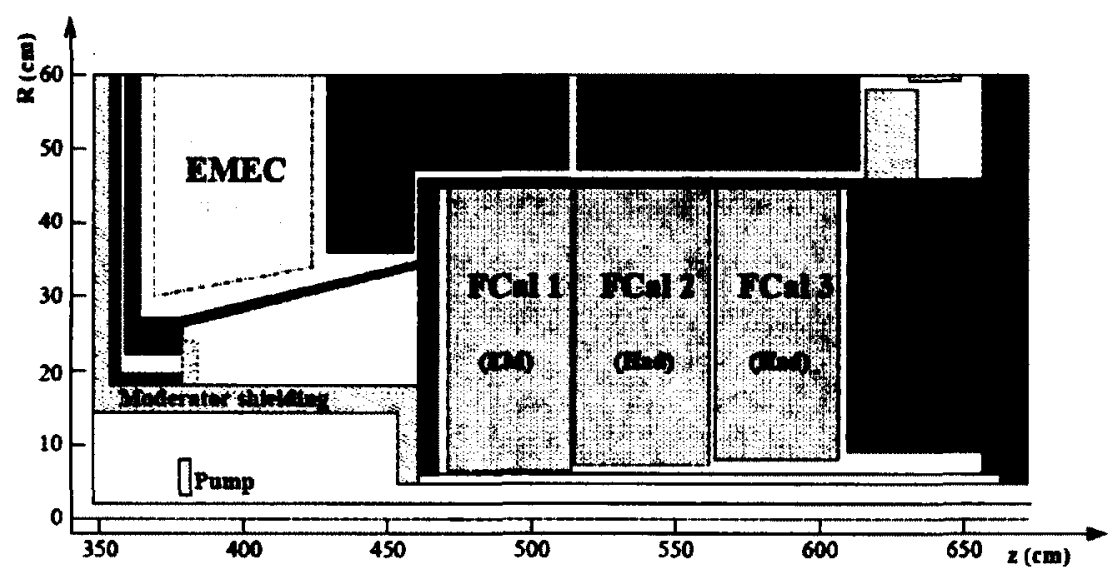

Figure 1.4: An FCal module. The interaction point is towards the left. [7]

of background noise in missing $E_{\mathrm{T}}$ measurements. The FCals provide energy and position measurements in the forward region, with design resolutions of better than 7\%. The FCals have been shown to provide an electron energy resolution of:

$$
\frac{\sigma_{E}}{E}=3.5 \% \bigoplus 28.5 \% \frac{\sqrt{G e V}}{\sqrt{E}},
$$

with an additional $1.2 \mathrm{GeV}$ of electronic noise and a pion energy resolution of:

$$
\frac{\sigma_{E}}{E}=7.5 \% \bigoplus 94 \% \frac{\sqrt{G e V}}{\sqrt{E}}
$$

with an additional $5.3 \mathrm{GeV}$ of electronic noise, where $\mathrm{E}$ is the energy of the incident electron or pion.

A full description of the FCal detector can be found in [16]. 


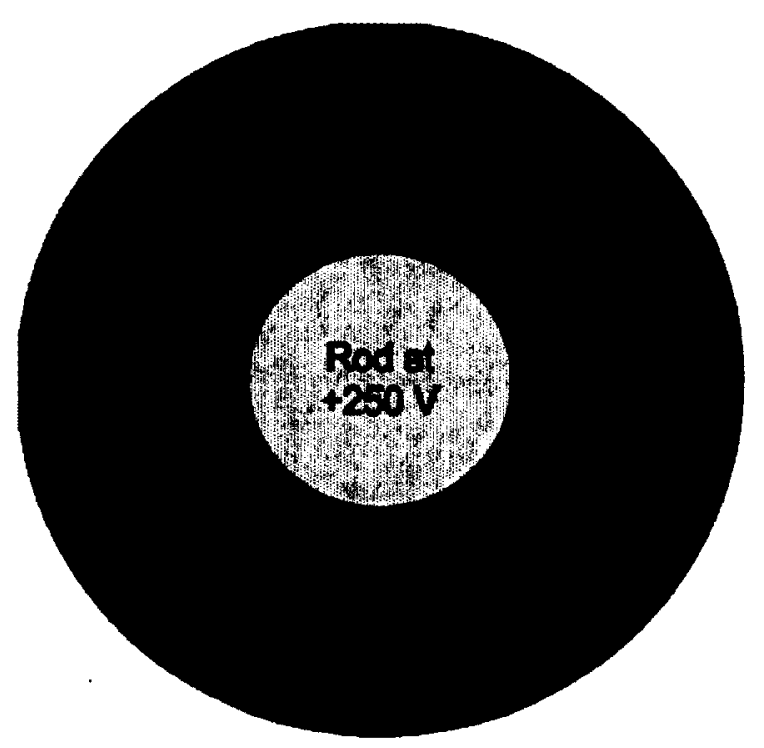

Figure 1.5: An FCal1 active gap. The rod and the absorber are copper in the FCal1 module and tungsten in the FCal2 and FCa/3 modules. The tubes are copper in all three modules. Note that the diagram is not to scale.

\subsubsection{Design}

Each FCal consists of three modules, as shown in Figure 1.4. Each uses liquid argon as its sampling material and is $444 \mathrm{~mm}$ deep with an outer radius of $449 \mathrm{~mm}$. The module closest to the interaction point (FCal1) has an absorber constructed of copper plates stacked one behind the other, and can be considered as an EM calorimeter. The FCal2 and FCal3 modules use tungsten for the absorber.

The FCall modules are 28 radiation lengths $\left(X_{0}\right)$, or 2.7 interaction lengths $(\lambda)$ deep, where $X_{0}$ is the amount of material necessary for a high energy electron to be reduced to $1 / \mathrm{e}$ of its original energy and $\lambda$ is the mean free path between nuclear interactions. The FCal2 and FCal3 modules are $3.7 \lambda$ and $3.6 \lambda$ deep respectively for a total active FCal depth of $10 \lambda$. Behind the FCal3 module is a 'plug' of passive brass (Plug 3), which helps shield the Muon Spectrometer. 
Due to their proximity to the beam pipe and the interaction point, the FCals are exposed to very high particle fluxes. Under these conditions, a liquid argon sampling gap of standard size $(2 \mathrm{~mm})$ would reach its critical ion density, where more argon ions are produced than collected, distorting the signal response of the electrode. To prevent this from occurring in the FCal, the electrodes are cylindrical structures that are parallel to the beam axis. Each electrode consists of an inner rod at high voltage and an outer tube kept at ground. The gap between them is maintained by a helically wound PEEK fibre and is filled with liquid argon. The electrode gaps in the FCal1 modules are approximately $0.25 \mathrm{~mm}$. The gaps in the FCal2 and 3 modules are a factor of 2 and 3 larger, respectively. This is possible due to the lower levels of particle flux at their locations. An illustration of the electrode structure is provided in Figure 1.5.

\subsection{High Luminosity Large Hadron Collider}

To improve the uncertainty on LHC measurements by a factor of two requires four times the statistics already gathered. Thus, running the LHC at the same luminosity for an extended period of time provides diminishing returns. To counteract this, plans to increase the luminosity at the LHC are already being developed. Dubbed the High Luminosity LHC (HL-LHC), the upgraded LHC has a target instantaneous luminosity of $5 \times 10^{34} \mathrm{~cm}^{-2} \mathrm{~s}^{-1}$ with a goal of achieving 10 times the integrated luminosity of the original LHC.

Since the ATLAS detector was built to operate at the LHC design luminosity, a five times increase in particle flux is expected to create some problems within existing detector systems. As a result, many portions of the ATLAS detector will likely require 
updating, including the FCal.

\subsubsection{Forward Calorimetry at the HL-LHC}

The FCals are expected to face three major problems at HL-LHC luminosities: the liquid argon gaps are expected to reach their critical ion density, reducing the electric field within the gaps and distorting their signal shape. The high voltage protection resistors used in the circuits that supply the FCal electrodes with a bias voltage are expected to draw increased current at the HL-LHC, reducing the voltage supplied to the electrodes. These problems compound one another; as the voltage supplied drops,

the number of uncollected ions increases. Early estimates predict nearly one third of the FCal1 module's pseudorapidity range will be compromised. Compromised regions in the other modules are unlikely to be as severe. Finally, finite element analysis (FEA) of the FCal at the HL-LHC design parameters predict a temperature increase in the liquid argon that, while not above argon's boiling point, provides very little safety margin.

The effect of these problems on the physics searches conducted at the LHC are currently being reviewed. If the signal loss in the FCal proves detrimental, a solution must be found. Two possible solutions are currently being explored. The first is the replacement of the FCals with a new calorimeter that is capable of operating at the HL-LHC design parameters, known as the super FCal ( $\mathrm{sFCal})$. The second is the insertion of a small, radiation hard calorimeter in front of the FCals, known as the Mini-FCal. The Mini-FCals are designed to absorb enough energy to allow the current FCals to operate at the HL-LHC. 


\subsubsection{Super FCal}

The design of the sFCal closely follows that of the original FCal, with minor modifcations. The liquid argon gaps must be decreased in size, down to $100 \mu \mathrm{m}$ in the new FCal1 modules. This gap size has been tested [17] and has been proven to successfully operate at the particle flux expected. The HV distribution circuits must be replaced with boards containing lower value HV protection resistors to avoid voltage drops at the electrodes. Finally, additional cooling loops must be installed in the vicinity of the sFCal to prevent excessive beam heating in the sFCals.

Installation of the $\mathrm{sFCals}$ requires the removal of the current FCals, which will have become highly activated. Although the exact nature of the process is still being discussed, at the very least some components of the end-cap cryostats will have to be removed and replaced to accomplish this. The time required to complete the installation is expected to be approximately 18 months.

\subsubsection{Mini-FCal}

The baseline Mini-FCal is a parallel-plate calorimeter that consists of copper absorber plates with alternating planes of semiconductor detectors, a depiction of which is shown in Figure 1.6. The detector planes consist of a substrate material with detectors mounted on both sides, providing good coverage. A wide range of semiconductor detectors had potential for use in the Mini-FCal. 3D silicon detectors, the most radiation hard of the silicon detectors, have been tested to the radiation levels expected within the ATLAS insertable B-Layer (IBL) at the HL-LHC [18][19], but have yet to be tested to the even higher radiation levels expected within the Mini-FCal. Initial tests of irradiated silicon carbide detectors produced conflicting results, likely due to 


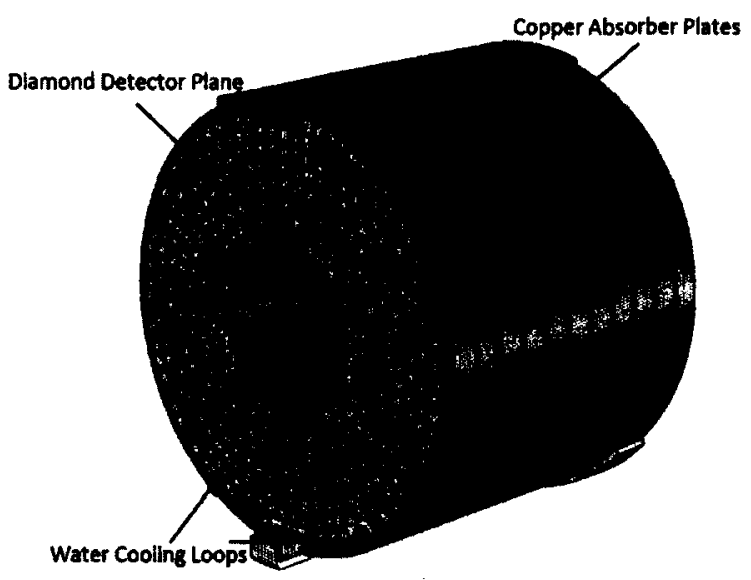

Figure 1.6: The Mini-FCal. The front absorber plate has been removed so that the first diamond detector plane is visible. Note that this is the original design sketch; the shape and layout of the diamond detectors has since been improved, and now provides better coverage.

the low quality of the initial materials [20]. Diamond detectors have been proven sufficiently resistant to survive the environment expected in the Mini-FCal (see Section 3). Diamond detectors have been used in many detector experiments as beam condition monitors, including the ATLAS and CMS experiments [21][22], and many others [23]. Plans exist to use diamond detector pixel modules in the IBL [24], as well as in future calorimeters [25], but have not been used in any existing calorimeters.

The Mini-FCal will be installed between the beam pipe and the inner warm wall of the end-cap cryostat, a depiction of which can be found in reference [26]. This region currently contains some neutron moderating material to prevent neutron backscatter from the FCals into the ID. The inner warm wall has an outer radius of $17.5 \mathrm{~cm}$ and a depth of $130 \mathrm{~cm}$, limiting the possible dimensions of the Mini-FCal. A depth of 10.5 radiation lengths (or $30 \mathrm{~cm}$ of copper) is deemed sufficient for the MiniFCal's purpose, leaving approximately $100 \mathrm{~cm}$ of space for a replacement neutron 
moderator in front of the Mini-FCal.

Preliminary simulations of the effect of introducing the Mini-FCal, carried out by Dr. Margret Fincke-Keeler, predict that the energy deposited in the inner regions of the FCall module would decrease by a factor of three, indicating that the Mini-FCal will allow the FCal1 module to operate at HL-LHC luminosities. Use of the Mini-FCal does, however, introduce an additional transition region at $|\eta|=4.0$, reducing the energy resolution in this region.

The installation of the Mini-FCal is relatively simple compared to replacing the FCal with the sFCal. The end-cap cryostat is capable of being moved approximately $5 \mathrm{~m}$ away from the IP, providing ample room to install the Mini-FCals from the IP end. From there, the neutron moderator can be removed and the Mini-FCals inserted directly, without opening the cryostats. In this case, the installation is predicted to take between six and eight months to complete. A preliminary FEA study has indicated that the tube should be capable of bearing the additional load of the MiniFCal, but some aspects of the FEA need to be cross-checked. Replacement of the support tube would undoubtedly increase the installation time.

$\mathrm{R} \& \mathrm{D}$ for the Mini-FCal project has discovered several drawbacks to the diamond detector option, which will be discussed further in Chapters 3 and 5. Additionally, the cost estimate for the diamond Mini-FCals has grown so that it is now on a par with the sFCal option. These factors have prompted exploration of alternative designs for the Mini-FCal. One such design is a high pressure xenon gas sampling calorimeter. $R \& D$ into this option is still in its preliminary stages, and is complicated by the fact that little prior research on the properties of high pressure xenon in a high rate environment has been undertaken. Another option is to use a liquid argon design similar to the sFCal. This is a convenient option in terms of both R\&D and cost, as 
liquid argon is a proven technology that would cost an order of magnitude less than the diamond option. A drawback to all of the Mini-FCal designs is the additional material required between them and the IP, which may create premature particle cascades. 


\section{Chapter 2}

\section{Fundamentals of Calorimetry and}

\section{Semiconductor Detectors}

The observation of particles is achieved via either their interactions in matter or, in the case of particles with very short decay times such as the $\pi^{0}$, the interactions of their decay products in matter. Different interactions dominate depending on the type of particle, with major differences between charged and neutral particles, of which photons are a special case. This chapter will detail how particles interact with matter and how their energy is reconstructed within a calorimeter, while specifically covering how the energy deposited by incident particles in a semiconducting material creates charge-carrier pairs, and how these pairs create a signal response via specialized readout electronics. 


\subsection{Particle Interactions in Matter}

The detection of a particle is based fundamentally on the interactions between the incident particle and the material through which it travels (termed the absorber material), specifically through the deposition of energy within the absorber. The amount of energy deposited is dependent upon numerous factors, the most important of which being the characteristics of the incident particle; charged and neutral particles behave differently when interacting with matter, with photonic interactions being a special case of neutral interactions. A more detailed treatment of these principles is provided in [1].

\subsubsection{Charged Particle Interactions}

Charged particles lose energy through repeated interactions with an absorber material. Thin absorber materials therefore experience large fluctuations in total energy deposited due to the inherently smaller number of interactions. The stopping power of a particle traveling through an absorber material is typically measured in $\langle d E / d x\rangle$, the mean energy deposited per unit length of material. Some authors use another measure, termed the mass stopping power, which is simply the stopping power divided by the material's density. It is also reported as $\langle d E / d x\rangle$.

The interactions leading to energy loss vary with the velocity of the incident particle. The interactions between charged particles and an absorber are predominantly electronic interactions. The energy loss of charged particles with 


\begin{tabular}{lll}
\hline \hline Symbol & Definition & Units or Value \\
\hline$E$ & Energy & $\mathrm{MeV}$ \\
$T$ & Maximum kinetic energy transfer & $\mathrm{MeV}$ \\
& $2 m_{e} c^{2} \beta^{2} \gamma^{2} /\left(1+2 \gamma \frac{m_{e}}{M}+\left(\frac{m_{e}}{M}\right)^{2}\right)$ & \\
$K$ & $4 \pi N_{A} r_{e}^{2} m_{e} c^{2}$ & $0.307075 \mathrm{MeV} \mathrm{cm}{ }^{2} / \mathrm{mol}$ \\
$N_{A}$ & Avogadro's number & $6.0221415(10) \times 10^{23} / \mathrm{mol}$ \\
$r_{e}$ & Classical electron radius & $2.817940325(28) \mathrm{fm}$ \\
& $e^{2} /\left(4 \pi \epsilon_{0} m_{e} c^{2}\right)$ & \\
$m_{e}$ & Electron mass & $0.510998918(44) \mathrm{MeV} / \mathrm{c}^{2}$ \\
$M$ & Mass of incident particle & $\mathrm{MeV} / \mathrm{c}^{2}$ \\
$z$ & Charge number of incident particle & \\
$Z$ & Atomic number of absorber & $\mathrm{g} / \mathrm{mol}$ \\
$A$ & Atomic mass of absorber & $\mathrm{eV}$ \\
$I$ & Mean excitation energy of absorber \\
$\delta(\beta \gamma)$ & Density effect correction & \\
\hline \hline
\end{tabular}

Table 2.1: Definition of variables used in the Bethe-Bloch equation

$0.1<\beta \gamma<1000$ can be described by the Bethe-Bloch equation [1]:

$$
-\left\langle\frac{d E}{d x}\right\rangle=K z^{2} \frac{Z}{A} \frac{1}{\beta^{2}}\left[\frac{1}{2} \ln \frac{2 m_{e} c^{2} \beta^{2} \gamma^{2} T_{\max }}{I^{2}}-\beta^{2}-\frac{\delta(\beta \gamma)}{2}\right] M e V c m^{2} / g
$$

where $\beta$ is the particle's velocity as a fraction of the speed of light and $\gamma$ is the particle's Lorentz factor. The other variables of this equation are listed in Table 2.1. The dominant source of energy loss described by the Bethe-Bloch equation is due to the ionization of the absorber material. For all practical purposes, energy loss 
in the Bethe-Bloch equation is dependent solely on the incident particle's velocity in the form of $\beta$ and $\gamma$, with slight dependences on the incident particle's mass at high energies (due to the $T_{\max }$ term) and the absorber materials atomic mass and number, in the form of $\mathrm{Z} / \mathrm{A}$. Since the majority of materials have a $\mathrm{Z} / \mathrm{A}$ of approximately 2 , this term has little actual effect.

Particles which lose the minimum amount of energy possible within the $\beta \gamma$ range of the Bethe-Bloch equation are said to be minimum ionizing particles (MIPs). MIPs deposit approximately $1.5 \mathrm{MeV} \cdot \mathrm{cm}^{2} / \mathrm{g}$ of energy into most materials. The amount of energy deposited by higher velocity particles varies slowly until radiative effects begin to dominate, which occurs at $O(10 \mathrm{MeV})$ for electrons and $O(100 \mathrm{GeV})$ for muons and pions. The onset of radiative effects scales with mass; for protons the onset occurs at much larger energies. Thus, MIPs are a useful approximation for ionization energy loss in all materials for charged particles.

The Bethe-Bloch equation is accurate within its range of validity to within a few percent. Corrections must be applied to accurately describe other $\beta \gamma$ values, such as the Anderson-Ziegler correction used for low energy corrections [27] and radiative corrections necessary to properly describe the energy loss of high energy particles [28]. The density effect correction [29][30][31] built into the Bethe-Bloch equation corrects for differences between solid and gaseous absorber materials at high velocities, prior to the onset of radiative effects. Finally, energy loss for particles with $\beta \gamma<$ $O(0.01)$ is dominated by electronic stopping power, which was successfully described by Lindhard and Scharff [32]. 


\subsubsection{Neutral Particle Interactions}

Neutral particles (other than the photon) lose energy predominately through direct collisions with nuclei, with the neutron being the most prevalent. Neutrons continue to travel through a material until enough energy loss has occurred for them to be completely absorbed. The energy transferred during these collisions is often enough to eject an atom from its crystal structure, creating lattice defects in crystalline materials.

\subsubsection{Photonic Interactions}

Photons interact with matter via three main channels: the photoelectric effect, Compton scattering, and pair-production. The interaction most relevant to forward calorimetry in ATLAS is pair-production, where a photon decays into an electronpositron pair. For a more detailed account of the other interactions, see reference $[1]$.

Photons become capable of pair-production when they have slightly more energy than two electrons at rest (1.022 MeV). However, at these energies Compton scattering is still the dominant interaction. At slightly higher energies $(O(10 \mathrm{MeV}))$, pair-production becomes the dominant interaction between photons and matter. Pairproduction of this form must occur within the field of a nucleus or electron to ensure conservation of energy and momentum. 


\subsection{Electromagnetic Cascades}

When a high-energy electron/positron or photon impinges upon an absorber material of sufficient thickness it initiates an EM cascade [33][34], creating lower energy electrons/positrons and photons via pair-production and bremsstrahlung radiation respectively. This process will repeat itself until too little energy remains to continue pair-production or bremsstrahlung radiation. The electron/positron pairs proceed to lose energy as per Section 2.1.1. This energy loss can be measured by a calorimeter and used to reconstruct the energy of the EM cascade.

\subsection{Hadronic Cascades}

High-energy hadron interactions with an absorber material are predominantly nuclear interactions. These interactions produce a wide variety of secondary particles, which initiate what is known as a hadronic cascade. A significant fraction of a hadron's interaction energy is transformed into an EM cascade via the decay of secondary $\pi^{0}$ 's and $\eta$ 's, which decay nearly instantly into photons. Charged secondaries deposit energy via ionization, but also interact with nuclei, producing neutrons and recoiling nuclei in highly excited states. Approximately $20 \%$ of a hadronic cascade's energy is contained within long-lived (with lifetimes measured in hundreds of ns) secondary neutrons. 


\subsection{Calorimetry}

Calorimeters are used to reconstruct the amount of energy possessed by a particle that impinges on them. To accomplish this, the calorimeter must contain enough material to completely contain the particle cascade(s) produced by the initial particle. The amount of material necessary to contain an EM cascade is measured in radiation lengths $\left(X_{0}\right)$. The number of radiation lengths required scales with $\ln (E)$, where $E$ is the energy of the incident particle, and is also dependent on the absorber material, but generally does not exceed $30 X_{0}$, or $43 \mathrm{~cm}$ of copper, even at LHC energies. Much more material is required to contain hadronic cascades. Measured in interaction lengths $(\lambda)$, hadronic cascades require approximately $10 \lambda$ of material, or $150 \mathrm{~cm}$ of copper, at LHC energies. More detail on both mechanisms can be found in reference [1].

Calorimeters can be further subdivided, based on their design, into either homogeneous or sampling calorimeters. Homogeneous calorimeters consist nearly entirely of active material, providing excellent energy resolution. Typical homogeneous calorimeters have an energy resolution of between $3-5 \% / \sqrt{E}$. They have yet to be used for hadronic calorimetry, as the volume necessary would not be cost-effective and the increased energy resolution is often wasted due to the energy lost to longlived neutrons (which generally deposit their energy outside the acceptance window of calorimeter electronics) produced during hadronic cascades.

Sampling calorimeters contain alternating regions of active material, such as scintillators, liquid noble gases, or semiconductors, and enough absorber material to contain particle showers of the maximum expected energy. To reduce the size of the calorimeter, the absorber material is usually a high-density material such as tungsten 
or copper. The energy resolution of a sampling calorimeter is dependent on several factors, including its sampling fraction, defined as the fraction of energy deposited in the active material of the calorimeter compared to the expected energy deposited within the entire calorimeter. The sampling fraction can be increased either by increasing the size of each active region or by increasing the number of active regions, averaging out the fluctuations in energy loss. At high-energy experiments such as ATLAS the energy resolution is dominated by calibration errors and non-uniformity in the calorimeter. Typical energy resolutions for EM sampling calorimeters range between $10-15 \% / \sqrt{E}$.

\subsubsection{Multiple Scattering}

A charged particle will undergo many small-angle scatters while traversing an absorber material. While large-angle scattering events are possible, they are sufficiently rare that a Gaussian approximation can be usefully applied, with the following result for the mean scattering angle, $\theta_{\text {plane }}^{\text {rms }}$ :

$$
\theta_{\text {plane }}^{\text {rms }}=\frac{1}{\sqrt{2}} \theta_{\text {space }}^{r m s}=\frac{13.6 M e V}{\beta c p} z \sqrt{x / X_{0}}\left[1+0.038 \ln x / X_{0}\right]
$$

where $p$ and $z$ are the momentum and charge number of the incident particle, respectively, while $x / X_{0}$ is the thickness of the absorber material in radiation lengths. This value is accurate to within $11 \%$ or better for $10^{-3}<x / X_{0}<100$. For a more complete treatment of multiple scattering, see reference [1]. 


\subsection{Properties of Semiconducting Detectors}

This section discusses the properties of semiconductors that are relevant to their use as particle detectors. As discussed in Section 2.1, particles traveling through matter deposit energy via a variety of mechanisms. The key feature of a semiconducting material that is important for detector physics is the presence of a small band-gap between a semiconductor's valence band of electrons and its conduction band.

Energy deposited through interactions with particles can free electrons from the valence band, leaving behind holes that behave as positively charged particles. This is known as either electron-hole (e-h) or charge-carrier production, depending on the author. Applying a bias voltage to electrodes attached to the semiconductor produces an electric field. The field attracts the charge-carriers, causing them to move towards the electrodes.

The movement of charge-carriers induces a current in the electrodes, which is amplified, and usually shaped, into a signal suitable for output. Since the amount of charge induced on the electrodes is proportional to the amount of charge-carriers created within the semiconductor, it is possible to predict the signal output of a particle of a particular energy (or vice versa) by calculating the charge induced on the electrodes by a charge-carrier at each point on the charge-carrier's trajectory. A much simpler way to accomplish this was independently developed by Shockley [35] and Ramo [36] for electrodes in vacuum tubes. The theory was later [37][38] proven valid in the presence of stationary space charge, extending its use to other materials, semiconductors included. 


\subsubsection{The Shockley-Ramo Theorem}

The Shockley-Ramo theorem states that the charge, $Q$, and current, $i$, induced on an electrode by a moving point charge, $q$, are given by:

$$
\begin{aligned}
Q & =-q \psi_{0}(x), \\
i & =q \boldsymbol{v} \cdot \boldsymbol{E}_{0}(x),
\end{aligned}
$$

where $\boldsymbol{v}$ is the instantaneous velocity of charge $q . \psi_{0}(x)$ and $\boldsymbol{E}_{0}(x)$ are the electric potential and field that would exist at $q$ 's instantaneous position, $x$, if the selected electrode was at unit potential, all other electrodes were at zero potential and all other charges were removed. Known as the weighting potential and field, respectively, the use of $\psi_{0}(x)$ and $\boldsymbol{E}_{0}(x)$ allows for a much easier calculation of the induced charge due to the potential's independence from the effects of stationary space charges and the moving charge itself.

Since a charge-carrier pair have opposite charges and move in opposite directions, their combined effect is additive; they produce the same sign current at a given electrode. If the charge-carrier pair travels the entire thickness of the detector, their combined effect is to induce a charge of $q$ on the electrode. This rarely occurs due to impurities in the detectors. These impurities can trap the charge-carriers, ending the induced current and reducing the total amount of charge induced. Complete charge collection is unlikely to occur except in very thin materials.

An important result of this theory is that the charge induced by $q$ is independent of the bias voltage applied to the electrodes and any stationary space charge present. Proof of this result, and a more thorough review of the Shockley-Ramo 
theorem in general, can be found in [39].

\subsubsection{Properties of Silicon Detectors}

The most extensively tested and widely used semiconductor detectors are based on silicon materials. To function effectively as a semiconductor, a junction of positively (p-type) and negatively (n-type) doped silicon is required. Combined, they create a region where charge-carrier production can be observed, known as the depletion region. The depletion region can expand or shrink based on the polarity and strength of a bias voltage applied to the electrodes.

Incident particles deposit energy and liberate charge-carrier pairs, as described in Section 2.5.1, throughout a silicon detector. However, only in the depletion region can the signal from charge-carrier pairs be observed above the background noise. To ensure that the maximum amount of signal is collected the depletion region must extend across the entire detector. A more detailed account of silicon detector mechanics can be found in [40].

\subsubsection{Properties of Diamond Detectors}

Current planar silicon detectors will not survive the conditions expected near the IP of the ATLAS detector over the lifetime of the HL-LHC [41]. This has prompted ongoing research into detector technologies for use in these high radiation regions, either by improving the current planar silicon technology [42] or, in the case of 3-D silicon detectors, by taking a different approach to the problem [43]. Silicon detectors benefit from an extensive, well developed infrastructure, making their production relatively cheap and of high quality. However, all junction based semiconductors are 
relatively weak to radiation damage. This has prompted $R \& D$ into the use of diamond as a detector material.

Some properties of interest for diamond and silicon are listed in Table 2.2. Of particular note are the differences in dielectric constant, band-gap energy, displacement energy, intrinsic carrier density and the number of charge-carrier pairs created per MIP.

Diamond's low dielectric constant gives it a lower capacitance than silicon. Since input capacitance contributes to background noise in preamplifier circuits, diamond detectors therefore experience lower levels of background noise. Diamond's high band-gap energy lowers the number of thermal electrons created, known as leakage current, which is another form of detector background noise. Another major factor in semiconductor noise levels is the material's intrinsic carrier density, which is low enough in diamond that it can be operated effectively without the use of a $\mathrm{p} / \mathrm{n}$ junction. Diamond's naturally high displacement energy results in a relatively high level of radiation hardness.

Diamond's high carrier mobility gives it a very fast charge collection time, generally on the order of $1 \mathrm{~ns}$ or less. Its low charge-carrier production does pose one disadvantage compared to silicon: a diamond detector would have to be twice as thick to produce the same magnitude signal. However, due to its naturally low noise level, its signal to noise (S/N) ratio is comparable (though currently inferior) to that of silicon [44].

Natural diamond is unsuitable for use in particle physics due to its size, rarity and concentration of impurities. Recent developments in the production of synthetic diamond has prompted the study of pure diamond in much greater detail. Due to 


\begin{tabular}{lllll}
\hline \hline Property & Symbol & Silicon & Diamond & Units \\
\hline Atomic number & $Z$ & 14 & 6 & \\
Density & $\rho$ & 2.33 & 3.52 & $\mathrm{~g} / \mathrm{cm}^{3}$ \\
Dielectric constant & $k$ & 11.9 & 5.7 & \\
Displacement energy & $E_{d}$ & 25 & 43 & $\mathrm{eV}$ \\
\hline Band-gap energy & $E_{g}$ & 1.12 & 5.48 & $\mathrm{eV}$ \\
Intrinsic carrier density & & $1.5 \times 10^{10}$ & $<10^{3}$ & $\mathrm{e} / \mathrm{cm}^{3}$ \\
Electron mobility & $\mu_{e}$ & 1450 & 1800 & $\mathrm{~cm} / \mathrm{V} \cdot \mathrm{s}$ \\
Hole mobility & $\mu_{h}$ & 505 & 1000 & $\mathrm{~cm} / \mathrm{V} \cdot \mathrm{s}$ \\
Mean e-h creation energy & $E_{e-h}$ & 3.61 & 13.1 & $\mathrm{eV}$ \\
\hline Thermal conductivity & $\lambda$ & 1.48 & $>1800$ & $\mathrm{~W} / \mathrm{K} \cdot \mathrm{cm}$ \\
Resistivity & $\rho$ & $10^{3}$ & $10^{13}$ & $\mathrm{k} \Omega \cdot \mathrm{cm}$ \\
Breakdown field & $X_{0}$ & 9.4 & 12.1 & $\mathrm{~cm}$ \\
\hline Mean energy loss per MIP & $\frac{d E}{d x}$ & 3.88 & 6.14 & $\mathrm{MVV} / \mathrm{cm}$ \\
Mean \# of e-h pairs created per MIP & & 108 & 47 & $\mathrm{e} / \mu \mathrm{m}$ \\
Radiation length & 0.3 & 0.5 & $\mathrm{MVm}$ \\
\hline \hline
\end{tabular}

Table 2.2: Material properties of silicon and diamond. Properties can be found throughout various sections of reference [1]. 


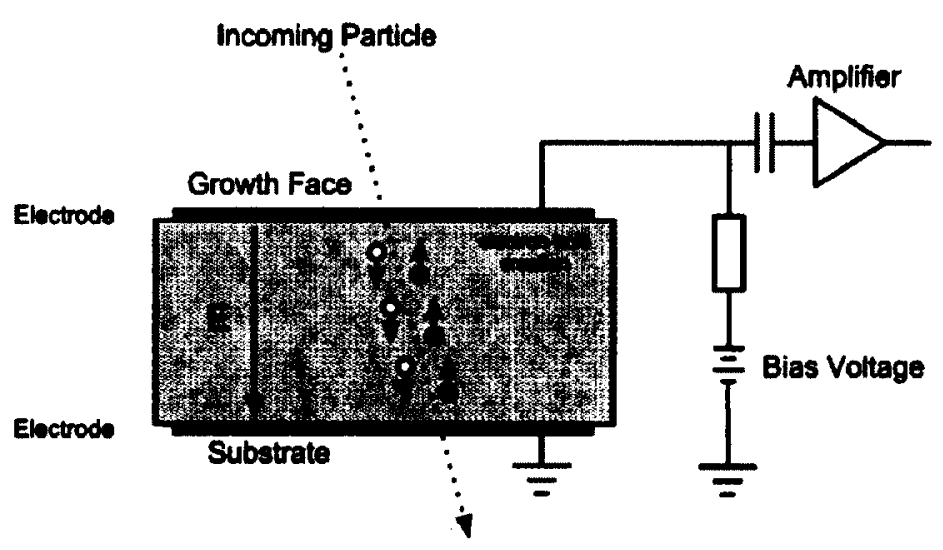

Figure 2.1: Depiction of charge collection in a CVD diamond detector.

limits on production infrastructure, it is still very expensive, leaving silicon as the material of choice for most semiconductor detector applications.

\subsubsection{Operation of a Diamond Detector}

A schematic of charge collection in a CVD diamond detector is provided in Figure 2.1. Metalization is applied to both the substrate and growth sides, providing electrical contacts through which a bias voltage can be applied. The bias voltage produces an electric field within the diamond which is used to collect the electron-hole pairs produced by ionizing particles traversing the detector. Unlike silicon detectors, a diamond detector does not require the use of a $\mathrm{p}-\mathrm{n}$ junction to produce a depletion region, due to diamond's naturally low intrinsic charge carrier density. The electronhole pairs induce a charge in the electrodes as they traverse the detector, which is amplified to produce a useful signal output. 


\subsubsection{Production of Synthetic Diamond}

Synthetic diamond is grown via a process called chemical vapour deposition (CVD). Carbon atoms are deposited from a microwave plasma [45] onto a substrate material. Care must be taken to suppress the growth of graphite layers while not preventing diamond growth as well, which can be accomplished by modifying the composition of the microwave plasma. Synthetic diamonds grow into either polycrystalline or single crystal structures, whose responses differ only in signal amplitude.

A pCVD diamond structure arises when multiple seed crystals begin growing on the substrate material during deposition. These crystals are initially very small. As more diamond accumulates, crystal size increases, with the largest crystals being found at the growth side of the detector. The boundaries between crystals, known as grain boundaries, act as trapping centres, capturing charge-carriers and reducing the signal response of the diamond. The concentration of grain boundaries decreases with growth and increases with growth rate, with the highest concentration of grain boundaries being found near the substrate material. For a depiction of the grain structure in pCVD diamond, see reference [46]. Growing the diamond slowly for an extended period of time and grinding away the substrate side are methods used to reduce the number of grain boundaries (and thus improve the signal response). pCVD diamond is produced in three grades, depending upon the density of grain boundaries (and thus the signal response) present within the diamond. From the lowest concentration of grain boundaries to the most, they are: electrical, optical, and polycrystalline.

scCVD diamond is formed similarly, but begins growth from a single seed crystal, eliminating the appearance of grain boundaries and providing better signal 
response [47]. It must be grown from specialized substrates, and is currently limited to approximately $1 \mathrm{~cm}^{2}$ of cross-sectional area [48]. The surface, or growth side, is uneven in both pCVD and scCVD diamond due to varying growth rates throughout the crystals, and usually requires grinding to provide uniform signal response. A depiction of charge collection in a CVD diamond detector is shown in Figure 2.1. Diamond Detectors Ltd. ${ }^{1}$ is currently the only commercial source of synthetic diamond detectors.

\subsection{Radiation Damage}

High levels of radiation are expected near the IP and in the forward region of the ATLAS detector. High particle fluxes cause considerable damage to both the active material and the readout electronics of a detector, resulting in a reduction in signal response and eventually detector failure. The damage caused by incident particles can be divided into two types: surface and bulk defects. Surface defects, such as those due to ionizing radiation damage in the detector metalization, create variations in the signal response of the detector, but are ultimately reversible and have little long-term effect on the detector.

The creation of bulk defects in the active material is the main concern for a particle detector. Crystalline lattice structures, such as those found in silicon and diamond detectors, as well as their readout electronics, are sensitive to radiation damage that results in lattice imperfections. Incident particles scatter off nuclei within the detector, knocking atoms out of their lattice positions, creating imperfections in the crystal lattice. A minimum of $25 \mathrm{eV}$ of energy must be transferred to accomplish

\footnotetext{
${ }^{1}$ DDL, 16 FleetBridge Business Centre, Upton Road, Poole, Dorset, UK, BH17 7AF
} 
this in silicon [49]. This can easily be accomplished by electrons and protons/neutrons with kinetic energies above 260 and $190 \mathrm{eV}$, respectively. The energies required in diamond materials are higher, requiring a minimum energy transfer of $43 \mathrm{eV}$. Compounding the problem, recoiling atoms often have enough excess energy to create additional lattice defects, creating volumes of localized defects.

A major concern for radiation damage is neutrons, the most common massive, neutral particle. While not as damaging as protons at low energies, neutrons are created in greater numbers in the forward region and are more difficult to contain. Neutrons are capable of penetrating much farther into a material than charged particles, and, as discussed in Section 2.1.2, interact mainly via direct collisions with the nuclei of atoms. This allows neutrons to cause an extensive amount of damage throughout a relatively large detector volume. Under irradiation, defect concentration continues to increase throughout the operation of a crystalline detector, eventually reaching the point where the bulk defects largely determine the material's properties.

The amount of damage caused by an incident particle is dependent both on the type and energy of the particle. These variations make it difficult to compare the damage caused by different particle types. To compare the effect of different particles on detectors, the damage must be scaled in some way. The current standard for silicon detectors is to use the Non-Ionizing Energy Loss (NIEL) hypothesis, which refers to any energy deposited into a material that wasn't due to a reversible ionization process, with the damage dealt by $1 \mathrm{MeV}$ neutrons used as a reference. The relative damage caused by various particle types and the conversion factors necessary to convert physical fluences into equivalent fluences can be found in reference [50]. NIEL scaling works well for most silicon materials. Although the trends observed in diamond materials are similar, NIEL scaling is not fully established for diamond 
materials. It is nevertheless an invaluable tool for the comparison of damage types in both materials.

\subsubsection{Effect of Radiation Damage in Semiconductor Detec- tors}

The effect of radiation damage in semiconductor detectors can be observed via changes in measurable quantities, particularly in the carrier lifetime and leakage current of the material. Since bulk defects provide additional sites for charge-carrier recombination, charge-carrier lifetime decreases as radiation damage accumulates. Due to the difficulty of directly measuring the charge-carrier lifetimes of a material, it is often more useful to measure the charge collection distance, $c c d$, given by:

$$
c c d=\tau_{e} v_{e}+\tau_{h} v_{h}=\left(\tau_{e} \mu_{e}+\tau_{h} \mu_{h}\right) E \approx \frac{Q}{Q_{0}} d,
$$

where $v, \tau$, and $\mu$ are the charge carrier's velocity, average lifetime, and mobility respectively, $E$ is the electric field within the material, $Q$ and $Q_{0}$ are the charge observed and the charge expected respectively, and $d$ is the detector thickness. The $c c d$ is simply the average distance traveled by the average charge-carrier. Since the signal response is directly proportional to the distance traveled by the charge-carriers, decreases in $c c d$ are observable via decreases in signal response, as seen in Figure 2.2.

It is possible to fill the trapping centres created by bulk defects by exposing the material to high levels of $\beta^{-}$radiation. This is known as pumping. This allows actual charge-carriers to travel further within the material, producing a larger signal response. The effect of pumping is characterized by an increase in signal response to a plateau, where the amount of charge being ejected has reached an equilibrium with 


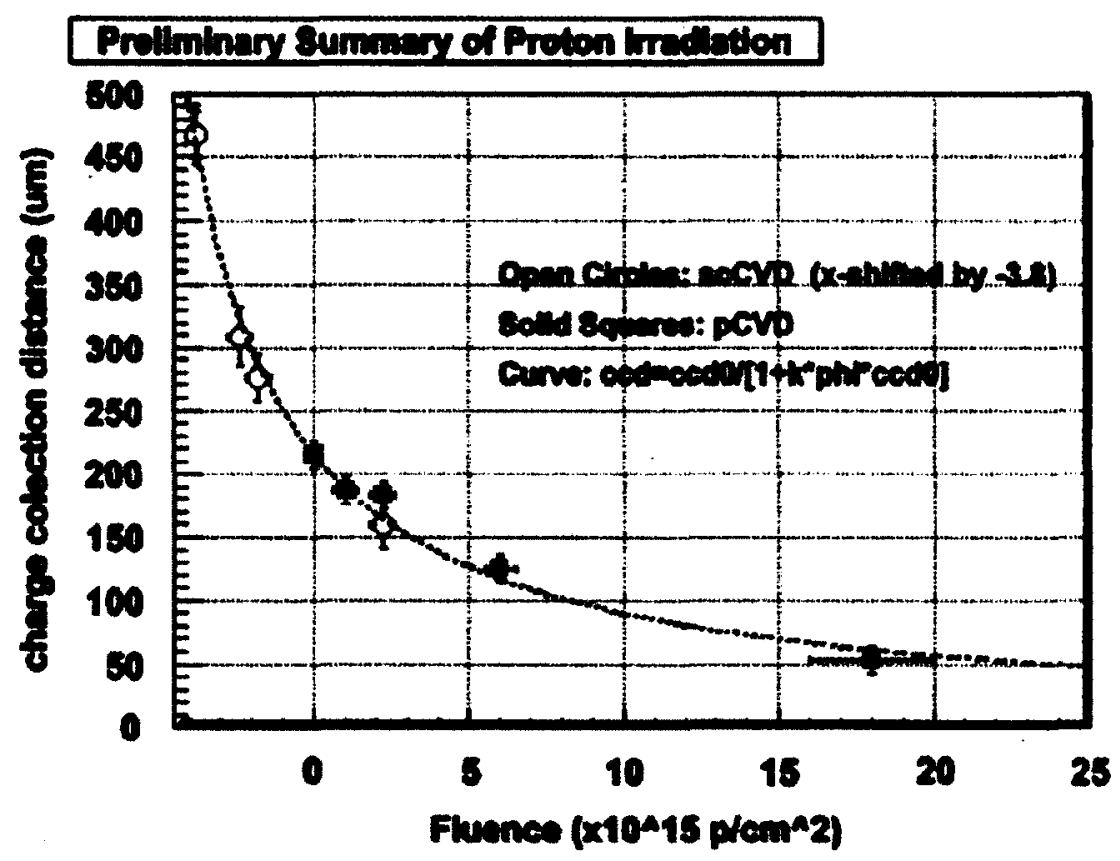

Figure 2.2: Summary of proton irradiation results for $p C V D$ (solid squares) and scCVD (open circles) diamond up to a fluence of $1.8 \times 10^{16}$ protons $/ \mathrm{cm}^{2}$ at proton energies of $24 \mathrm{GeV}$. The data are fit to a damage curve $\left(1 / c c d=1 / c c d_{0}+k \phi\right)$, shown in black. The scCVD data have been shifted to the left by a fluence of $3.8 \times 10^{15}$ protons $/ \mathrm{cm}^{2}$ to match the $p C V D$ data. This indicates that $p C V D$ and $s c C V D$ diamond share a common damage coefficient and that the grain boundaries present within $p C V D$ diamond behave similarly to radiation damage [51]. 
the amount of charge being captured.

Radiation damage creates additional energy levels between the valence and conduction bands of a semiconductor, reducing the amount of energy required for a thermal electron to be created and increasing the amount of leakage current within a detector. The increase in leakage current, $I_{l}$, with particle fluence, $\phi$, for a fully depleted silicon detector is given by:

$$
I_{l}=I_{0}+\alpha \phi V,
$$

where $I_{0}$ is the leakage current prior to irradiation, $V$ is volume of the detector, and $\alpha$ is the damage coefficient, which depends on the absorber material. For silicon detectors, a value of $\alpha=4.0 \times 10^{-17} \mathrm{~A} / \mathrm{cm}$ has been found [52]. Diamond detectors have shown no increase in leakage current post irradiation [53], likely due to their low intrinsic charge-carrier density and large band gap energy. Increased leakage current reduces the $\mathrm{S} / \mathrm{N}$ ratio of a detector, and can eventually make a silicon detector inoperable. 


\section{Chapter 3}

\section{Irradiation of pCVD Diamond}

\section{Detectors}

The Mini-FCal is expected to receive an integrated particle flux in excess of $2 \times$ $10^{17}$ neutrons $/ \mathrm{cm}^{2}$ in ten years of HL-LHC operation. To determine whether pCVD diamond detectors are capable of sustained operation at this integrated flux, four detectors from DDL were placed in the proton beam from the TRIUMF ${ }^{1}$ cyclotron in September 2010 and irradiated over the course of 12 days. While proton irradiation is not equivalent to neutron irradiation and the NIEL hypothesis is not fully established for diamond, it is much easier to achieve a constant energy proton beam, making it a useful comparison.

This chapter will briefly describe the basics of this test and its results. A complete analysis of this test can be found in [54].

\footnotetext{
${ }^{1}$ TRIUMF, Canada's National Laboratory for Particle and Nuclear Physics, 4004 Wesbrook Mall, Vancouver, BC, Canada, V6T 2 A3.
} 


\begin{tabular}{llll}
\hline Detector & Thickness & Grade & Metalization \\
\hline 1 & $200 \mu \mathrm{m}$ & Electronic & TiW-Al \\
2 & $300 \mu \mathrm{m}$ & Optical & TiW-Al \\
3 & $300 \mu \mathrm{m}$ & Electronic & $\mathrm{Au}-\mathrm{Pt}$ \\
4 & $300 \mu \mathrm{m}$ & Optical & $\mathrm{Au}-\mathrm{Pt}$ \\
\hline
\end{tabular}

Table 3.1: Properties of diamond detectors used in the irradiation test at TRIUMF. [54]

\subsection{Introduction}

The detectors studied were $8 \mathrm{~mm}$ in diameter and either 200 or 300 microns thick. The detectors were not polished prior to the metalization process, to match the detectors that will be used in the Mini-FCal. The metalization and grade of each detector varied, providing an additional avenue of investigation. The properties of each detector can be found in Table 3.1. Since the irradiation occurred within a vacuum, it was unclear whether the diamonds would radiate enough heat to remain operational. Thermocouples were therefore attached to the detectors to measure their temperature throughout the experiment. The cyclotron produced $500 \mathrm{MeV} / \mathrm{c}$ proton bunches at a frequency of $23 \mathrm{MHz}$ with a duty cycle of $90 \%$. The detectors were placed in the beamline immediately downstream of three sets of secondary emission chambers, used to monitor the proton beam position and measure the proton flux. An aluminum activation foil was placed immediately downstream of the detectors to provide an independent measurement of the particle flux.

Earlier diamond irradiation experiments have shown a fast decay in signal response during initial irradiation, as shown in Figure 2.2. To more accurately measure the initial signal decay, the experiment began at a proton current of $4 \mathrm{nA}$, the min- 


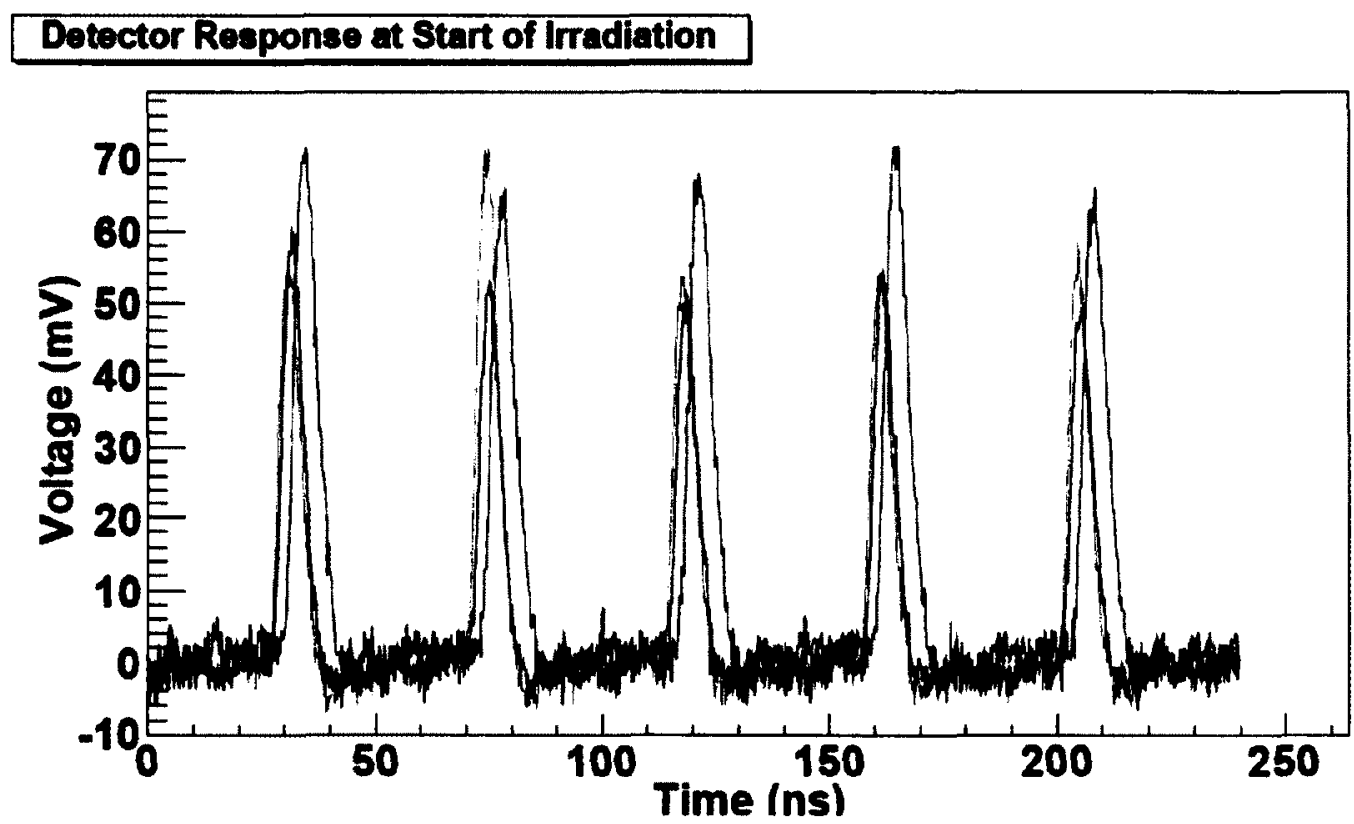

Figure 3.1: Response of three diamond detectors at the beginning of the test. Each pulse displays the detector response to a cyclotron pulse of approximately 800 protons. [54] 
imum stable beam current that could be achieved, and was later increased to $20 \mathrm{nA}$ and then $40 \mathrm{nA}$. The charge collected in each diamond was amplified using custom preamplifiers near the beamline. A four-channel oscilloscope was used to sample the response of three of the detectors and the cyclotron radio frequency clock with a period of $0.2 \mathrm{~ns}$ for 100,000 samples. These measurements were recorded every $30 \mathrm{~s}$. Detectors one and two were monitored continuously, while measurements of detectors three and four were alternated every hour. An example of the detector response can be seen in Figure 3.1. The time stamp, bias voltage, summed current to the four detectors, and the four thermocouples were also recorded every $30 \mathrm{~s}$ for use in the analysis and to ensure data quality.

\subsection{Results}

The signal response as a function of fluence (integrated flux) for all four detectors is shown in Figure 3.2. Each point is constructed by averaging the signal integral of 120 radio frequency periods after the pedestal has been removed and is normalized to the proton current extracted from the secondary emission chambers.

The response of all four detectors decreased to approximately $5 \%$ of their original response after exposure to $2.25 \times 10^{17}$ protons $/ \mathrm{cm}^{2}$. The TiW-Al metalized detectors appear to operate slightly better than their counterparts after high levels of irradiation. No difference between detector grades was observed during this test. The thermocouples registered no increase in temperature throughout the test. These results are consistent with earlier measurements from the RD42 working group [51].

The aluminum activation foil was used to verify the secondary emission chamber's integrated flux reading. The average integrated flux obtained by counting an 8 


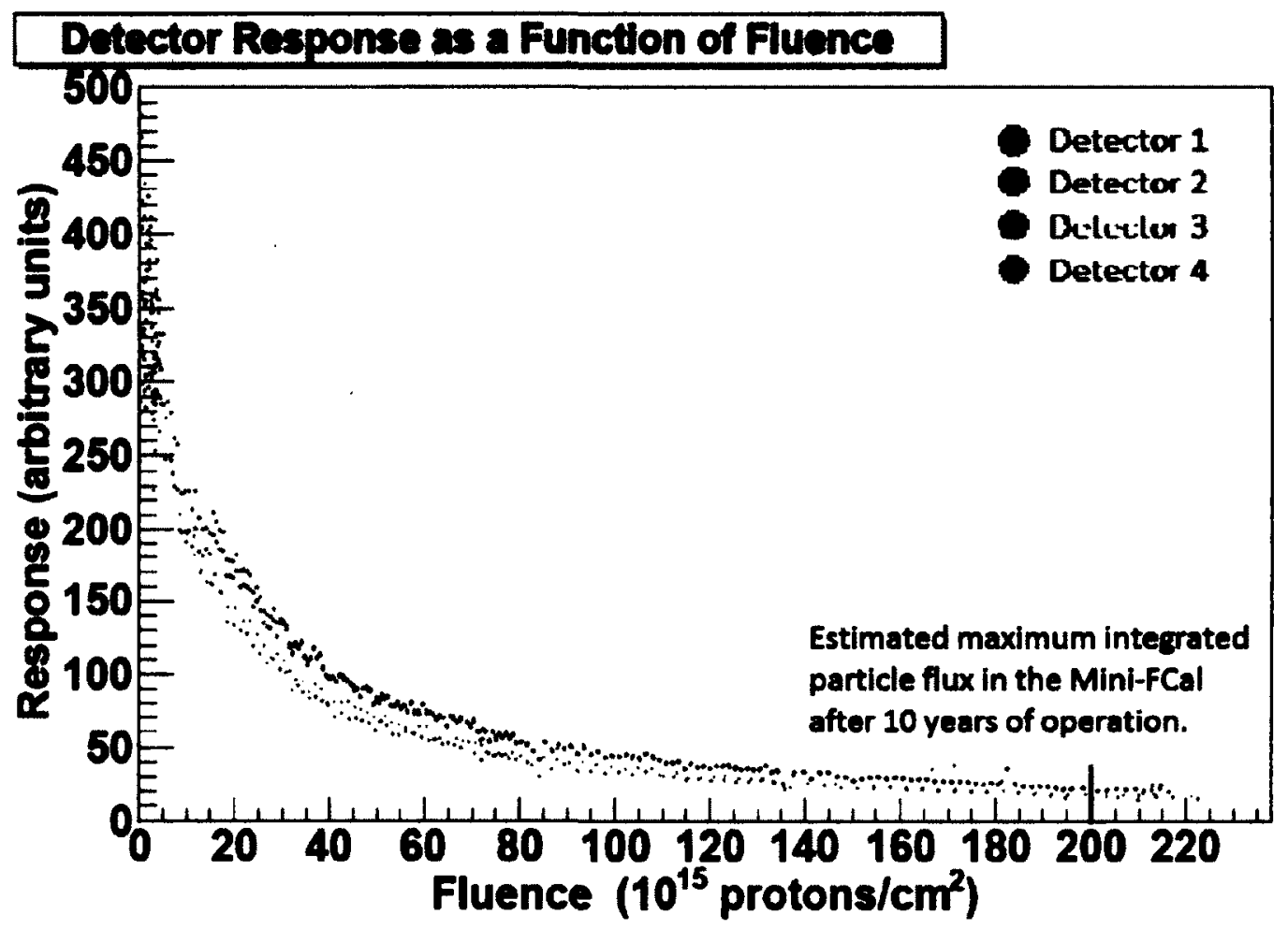

Figure 3.2: Response of the four detectors as a function of fluence. From top to bottom, detectors are: 1, 2, 3, 4, as noted in Table 3.1. [54] 
$\mathrm{mm}$ disk aligned with the detectors was $(2.09 \pm 0.06) \times 10^{17}$ protons $/ \mathrm{cm}^{2}$ from ${ }^{22} \mathrm{Na}$ counting and $(2.47 \pm 0.10) \times 10^{17}$ protons $/ \mathrm{cm}^{2}$ from ${ }^{7} \mathrm{Be}$ counting, for an average integrated flux of $2.28 \times 10^{17} \mathrm{protons} / \mathrm{cm}^{2}$, which is consistent with the integrated flux measured by the secondary emission chambers.

Beam profile plots were obtained by counting a series of $2 \mathrm{~mm}$ diameter disks. The integrated flux at the centre of the detectors was measured to be $(5.14 \pm 0.22)$ $\times 10^{17}$ protons $/ \mathrm{cm}^{2}$ from ${ }^{22} \mathrm{Na}$ counting and $(5.29 \pm 0.30) \times 10^{17}$ protons $/ \mathrm{cm}^{2}$ from ${ }^{7} \mathrm{Be}$ counting.

\subsection{Summary of Irradiation Test}

This irradiation test showed that pCVD diamond detectors are capable of operating at integrated fluxes in excess of $5 \times 10^{17}$ protons $/ \mathrm{cm}^{2}$, although at a vastly reduced signal level. This integrated flux corresponds to the levels expected within the MiniFCal at the HL-LHC. Both metalizations commonly applied by DDL survived the radiation levels as well, with TiW-Al providing higher signal levels than Au-Pt. The differences between electrical and optical grade detectors appears to be negligible at these radiation levels, although irradiation tests of additional detectors would be advised to verify this result. Due to the inaccuracies of the NIEL hypothesis in diamond, confirmation that these results are applicable to neutron irradiation is required. Experiments with a neutron beam are planned for 2012 at the Dubna reactor in Russia, which will help determine whether proton irradiation results can be scaled and applied to simulate neutron irradiation in diamond. If so, the proton irradiation results from TRIUMF will be used to determine the energy corrections necessary to accurately measure energy in the Mini-FCal at the HL-LHC. 


\section{Chapter 4}

\section{Characterization of pCVD}

\section{Diamond Detectors in Preparation}

\section{for Spatial Uniformity}

\section{Measurements}

The baseline design of the Mini-FCal requires the use of over 8000 diamond detectors. These detectors will be made of unpolished pCVD diamond of optical or electrical grade. The numerous grain boundaries present within pCVD diamond, as discussed in Section 2.5.5, create variations in signal response throughout the bulk of the detector. This section describes a test to quantify these variations, as well as the laboratory tests that were required in preparation for the test. These measurements will be used in simulations of the Mini-FCal response, providing an improved estimate of its performance. 


\begin{tabular}{llll}
\hline \hline Detector & Thickness & Grade & Metalization \\
\hline $\mathrm{B}$ & $700 \mu \mathrm{m}$ & Electronic & $\mathrm{Au}-\mathrm{Pt}$ \\
1 & $800 \mu \mathrm{m}$ & Optical & $\mathrm{Au}-\mathrm{Pt}$ \\
3 & $600 \mu \mathrm{m}$ & Electronic & $\mathrm{Au}-\mathrm{Pt}$ \\
7 & $600 \mu \mathrm{m}$ & Polycrystalline & $\mathrm{TiW}-\mathrm{Al}$ \\
\hline \hline
\end{tabular}

Table 4.1: Properties of diamond detectors available for use in spatial uniformity measurements.

This chapter will describe the characteristics of the diamond detector studied in the test, the silicon detector used to measure the position of the beam particles in the diamond detector, and finally the spatial uniformity test.

\subsection{Diamond Detector Properties}

Four detectors were available for laboratory measurements and use in the spatial uniformity test. Each detector consisted of an unpolished diamond with a surface area of $1 \mathrm{~cm} \mathrm{x} 1 \mathrm{~cm}$ and a thickness ranging from $600 \mu \mathrm{m}-800 \mu \mathrm{m}$. Metalization was applied to the two largest surfaces in $8 \mathrm{~mm}$ diameter circles. Other properties of the diamond detectors can be found in Table 4.1. All were pCVD detectors provided by DDL, three of which had recently been remetalized (detectors 1,3 , and 7). Detectors were returned mislabeled as detectors 1,2 , and 6 . As such, there is some uncertainty as to the exact properties of a specific detector.

A MIP will create on average 4000 charge carrier pairs per $100 \mu \mathrm{m}$ of diamond traversed. Amplifiers are required to convert induced charge into a usable signal. 
Cremat ${ }^{1}$ amplifiers were chosen for this purpose.

Cremat produces several charge sensitive preamplifier modules, which differ primarily in their gain, and a series of seven Gaussian shaping modules, which differ only in their shaping time. Given the low signal levels expected from the diamond detectors, the charge sensitive preamplifier with highest gain, the CR-110, was chosen. The fastest shaper, the CR-200-100ns, was also chosen, which gives a pulse width of approximately $250 \mathrm{~ns}$ and allows the amplifier to effectively shape 4 particles/ $\mu \mathrm{s}$.

Three identical amplifiers were built for use in the spatial uniformity test. Each consisted of a CR-110 module mounted on a CR-150 evaluation board and a CR-200 module mounted on a CR-160 evaluation board. For detailed schematics of the modules and evaluation boards, see [55]. Both evaluation boards are mounted within an aluminum housing, as shown in Figure 4.1. The Cremat amplifiers ideally produce a pulse amplitude of approximately $50 \mathrm{mV}$ per $100 \mu \mathrm{m}$ of diamond traversed by a MIP.

All three identical Cremats were tested extensively and showed little to no difference in response during laboratory tests.

\subsubsection{Laboratory Tests}

Studies of the diamond detectors in a laboratory setting were conducted prior to the spatial uniformity test. The detectors were positioned above two scintillators, which were used for trigger purposes. The scintillator signals were discriminated and passed through a NIM logic unit, which would trigger an oscilloscope ${ }^{2}$ to measure the

\footnotetext{
${ }^{1}$ Cremat, Inc., 45 Union St., Watertown, MA 02472
}

${ }^{2}$ Agilent Technologies Infiniium DSO9404A 


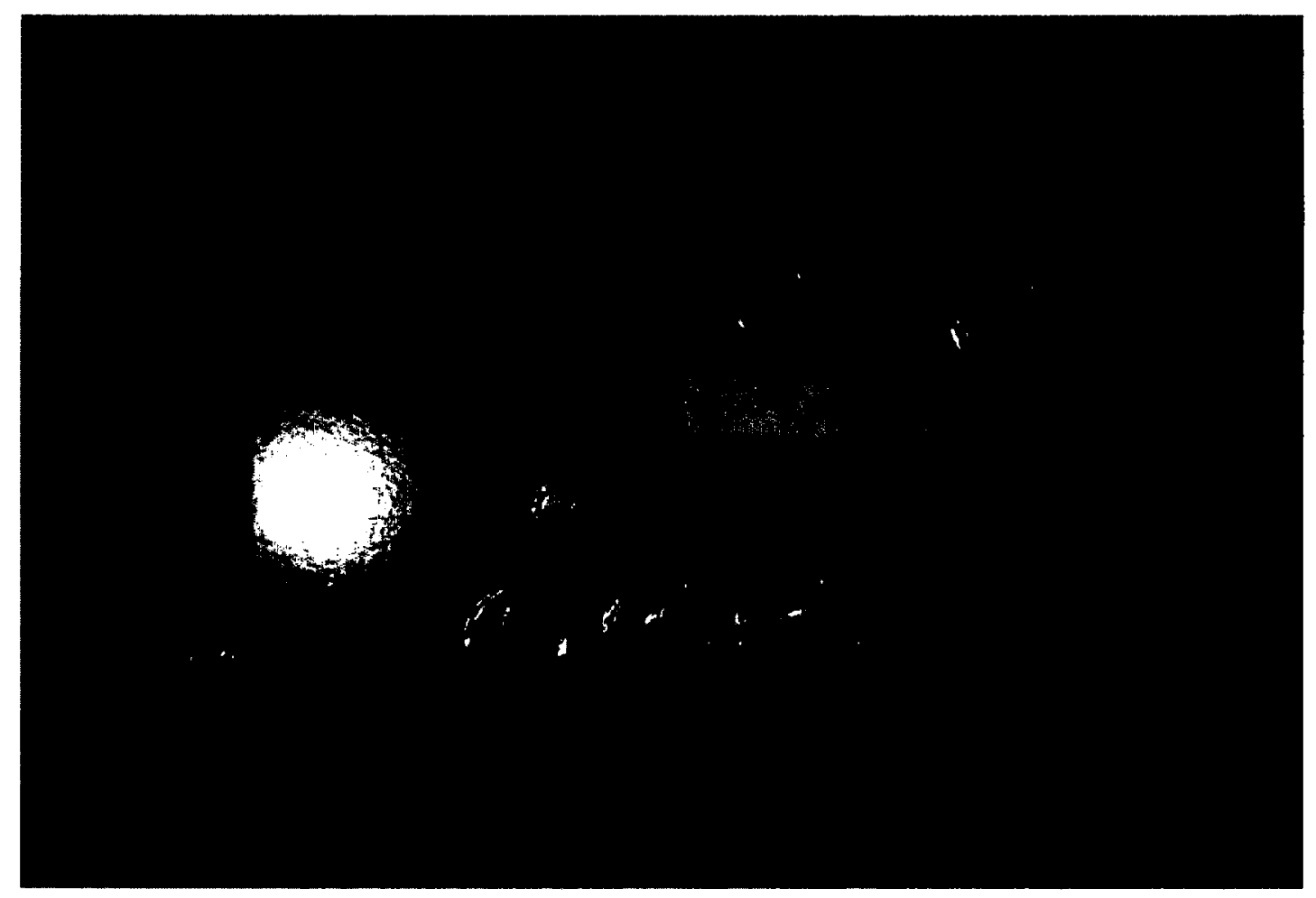

Figure 4.1: Cremat amplifier used for spatial uniformity measurements. The connector labeled BIAS is used to supply a HV to the diamond detector, whose connector can be seen on the far left. The TEST connector can be used to supply a known voltage to the preamplifier board, to test its response. The E OUT, SHAPE IN, and SHAPE OUT connectors are the output from the preamplifier board, and the input and output to the Gaussian shaper board, respectively. The opening labeled GAIN allows access to the shaper board's fine-tuner, which can be set anywhere from 0 to $100 \%$ gain. The connector used to supply the $L V$ necessary for the amplifier to operate is located on the right side of the housing, not shown. 


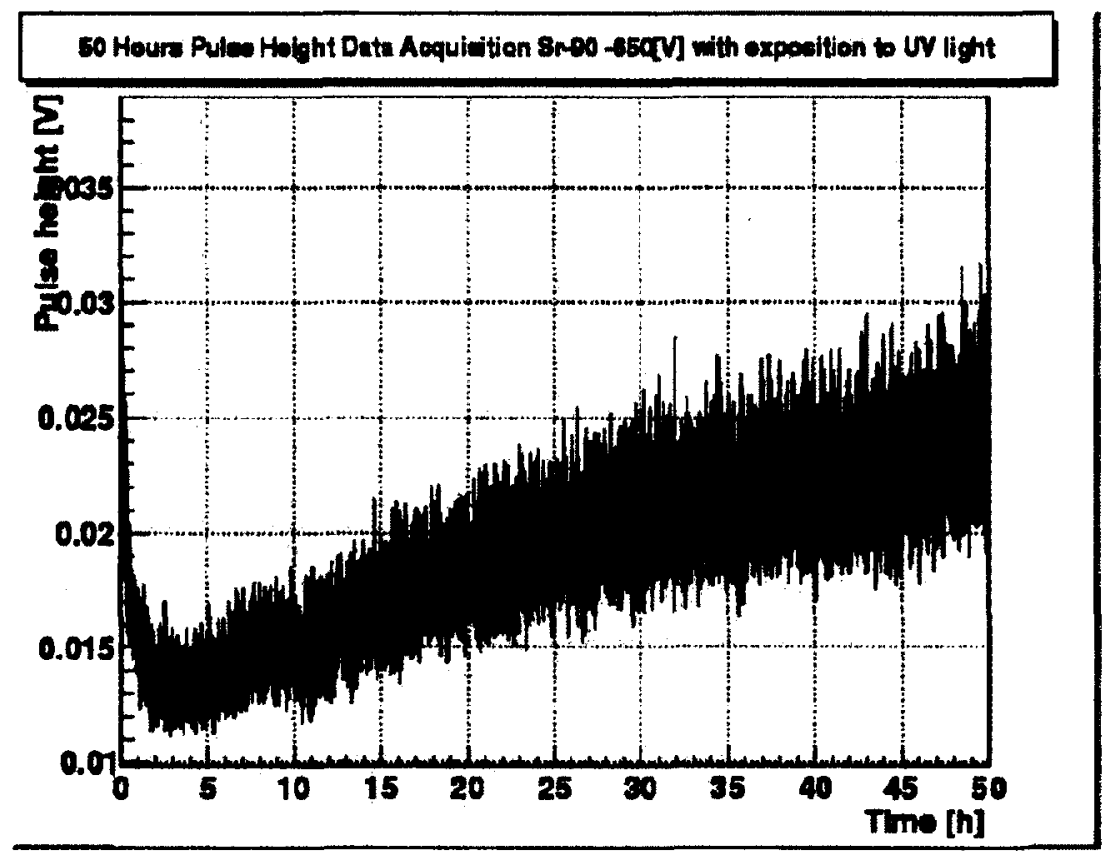

Figure 4.2: Response of diamond detector $B$ after exposure to UV radiation. The pulse height is initially $30 \mathrm{mV}$ and decays to approximately $12 \mathrm{mV}$ over the course of two hours. The response slowly climbs back to the $30 \mathrm{mV}$ level over the course of 48 hours. This is consistent with the pumping effect.

corresponding diamond waveform if the scintillator signals were in coincidence. The majority of tests used a Strontium-90 $\left({ }^{90} \mathrm{Sr}\right)$ beta source to provide incident particles and were analyzed using LabVIEW ${ }^{3}$ programs.

Detector B arrived in the laboratory well before detectors 1,2 , and 6 , and as such was studied much more extensively. It was first characterized by a summer student, Charles-Antoine Collins Fekete, who observed the pumping effect expected of diamond detectors. Exposure to UV light ejects electrons from diamond detectors, creating additional charge-carrier trapping sites and thus reducing the signal response

\footnotetext{
${ }^{3}$ National Instruments Canada, 1000 Avenue Saint-Charles, Suite 812, Vaudreuil-Dorion, Quebec Canada J7V 8P5
} 
of the detectors. Exposure to a beta source fills the trapping sites, increasing the signal response of the detectors until a plateau is reached, as shown in Figure 4.2. Note that the initial fast decay in signal response may be due to polarization effects within the diamond detector caused by changes in the applied bias voltage.

The I-V curve is a simple procedure for characterizing detectors. In this test, a series of voltages are supplied to the detector and the resulting leakage current is measured. A semiconductor, such as diamond, should show a quick rise in current with increasing voltage, followed by a plateau region and, eventually, another quick rise. A voltage in the plateau region can be considered to be the detector's optimal operating voltage. A second quick rise in leakage current at higher voltages denotes the breakdown voltage of the detector. Voltages in this region may damage the detector. Standard diamond detectors have a breakdown voltage of approximately 50 $\mathrm{V}$ per $\mu \mathrm{m}$ of detector thickness, while the standard operating field is $1 \mathrm{~V} / \mu \mathrm{m}$.

To obtain the I-V curves the voltage applied to the detectors was increased in steps of $10 \mathrm{~V}$ to their standard operating voltage. The leakage current produced took approximately 15 seconds to stabilize between steps. No data was taken during this time, as it is not indicative of the detector response. The results are shown in Figures 4.3 - 4.6. The expected behaviour was observed in detectors B and 1, with plateau regions extending from 200 and $300 \mathrm{mV}$ to the maximum voltage applied, respectively. Neither detector 2 or 6 show the characteristic plateau region of a semiconductor, with detector 6 clearly reaching a breakdown field at a bias voltage of $400 \mathrm{~V}$. Neither detector produced a measurable signal response from a ${ }^{90} \mathrm{Sr}$ source. These detectors were once again returned to DDL for repairs. The signal response of detectors B and 1 were then examined, with charge collection distances of $77 \mu \mathrm{m}$ and $21 \mu \mathrm{m}$ respectively. As such, detector B was chosen for use in the spatial uniformity 


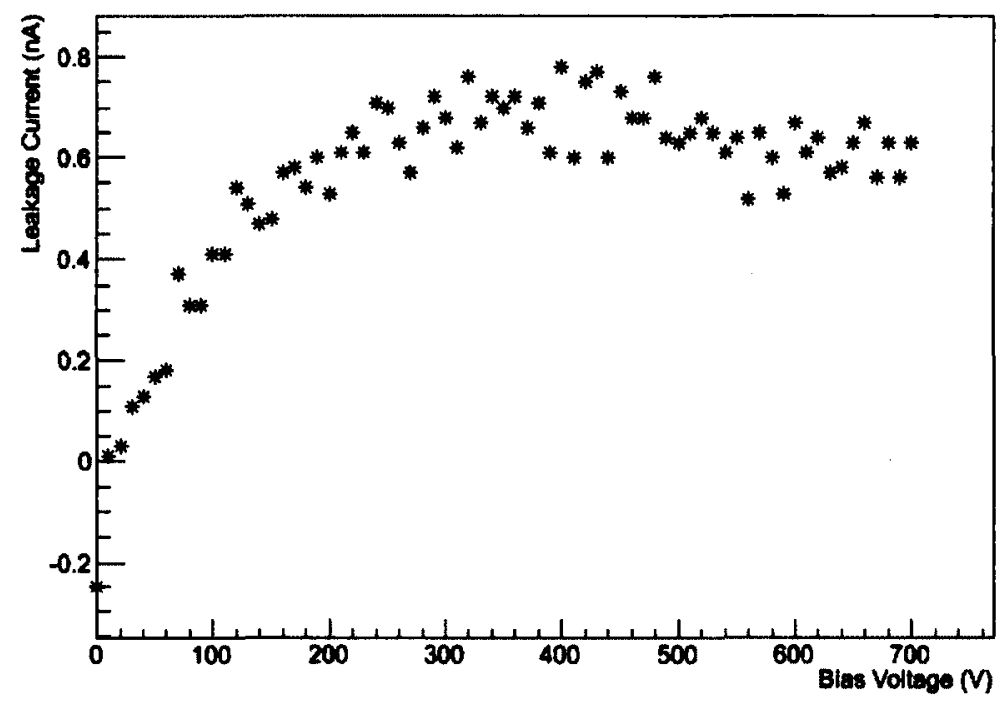

Figure 4.3: Leakage current produced by diamond detector $B$ under varying applied bias voltages. The leakage current plateau is consistent with the behaviour expected of a semiconductor.

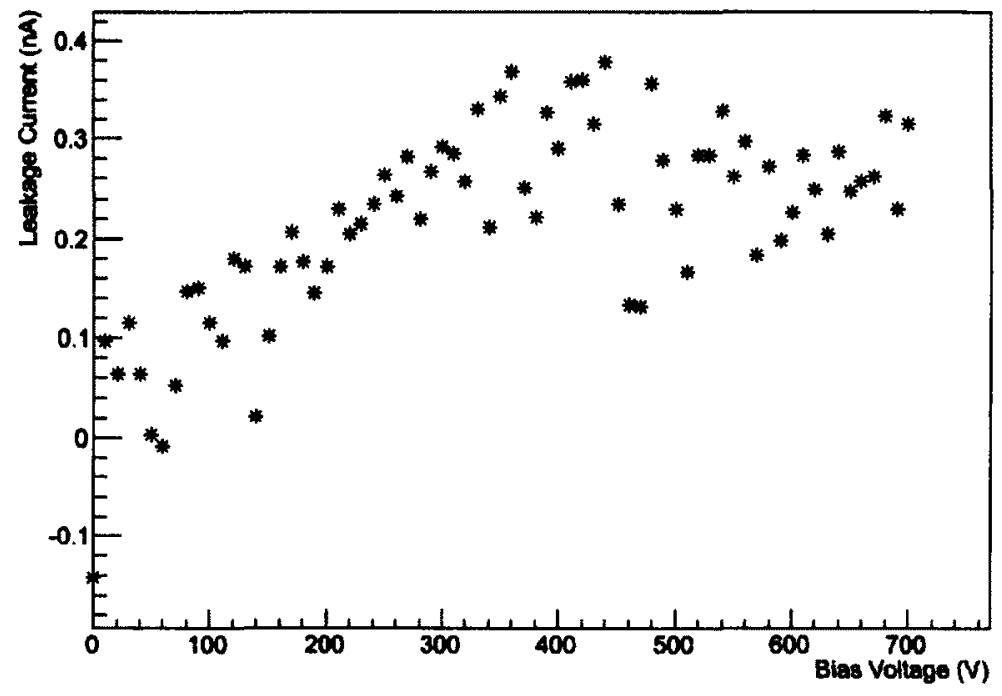

Figure 4.4: Leakage current produced by diamond detector 1 under varying applied bias voltages. 


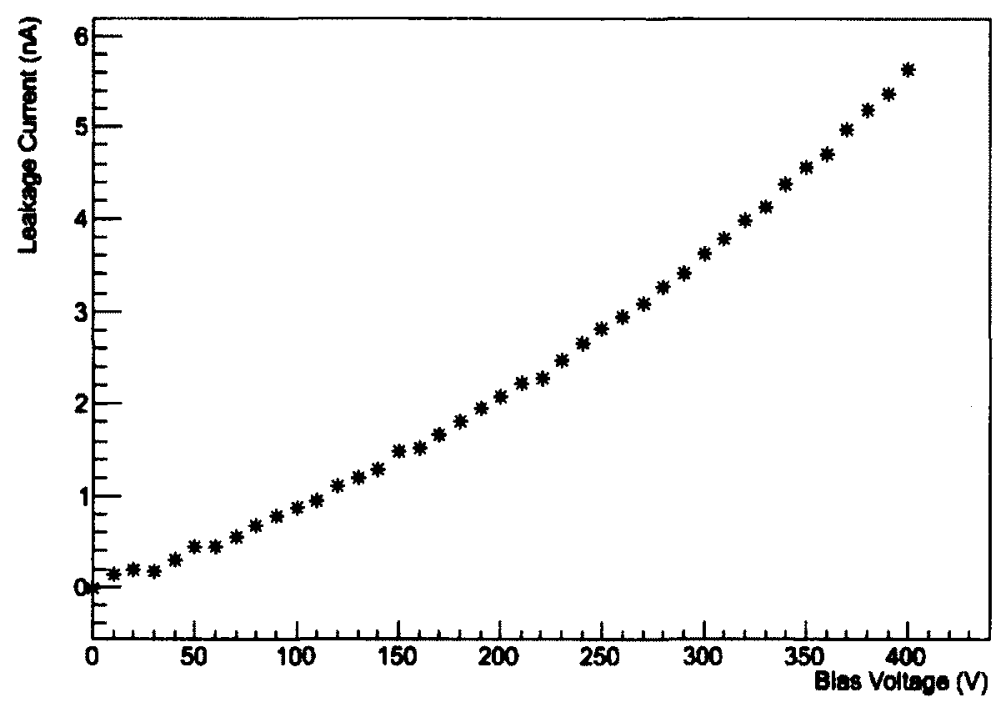

Figure 4.5: Leakage current produced by diamond detector 2 under varying applied bias voltages. The response is inconsistent with the behaviour expected of a semiconductor, with no discernible plateau region.

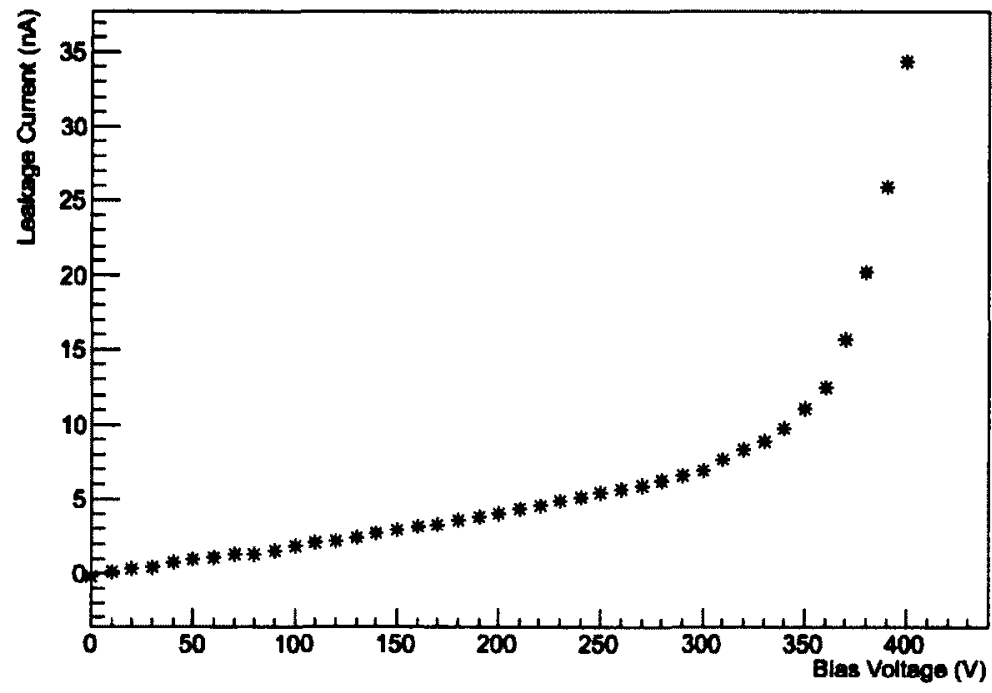

Figure 4.6: Leakage current produced by diamond detector 6 under varying applied bias voltages. The detector reaches its breakdown field by $400 \mathrm{~V}$. 


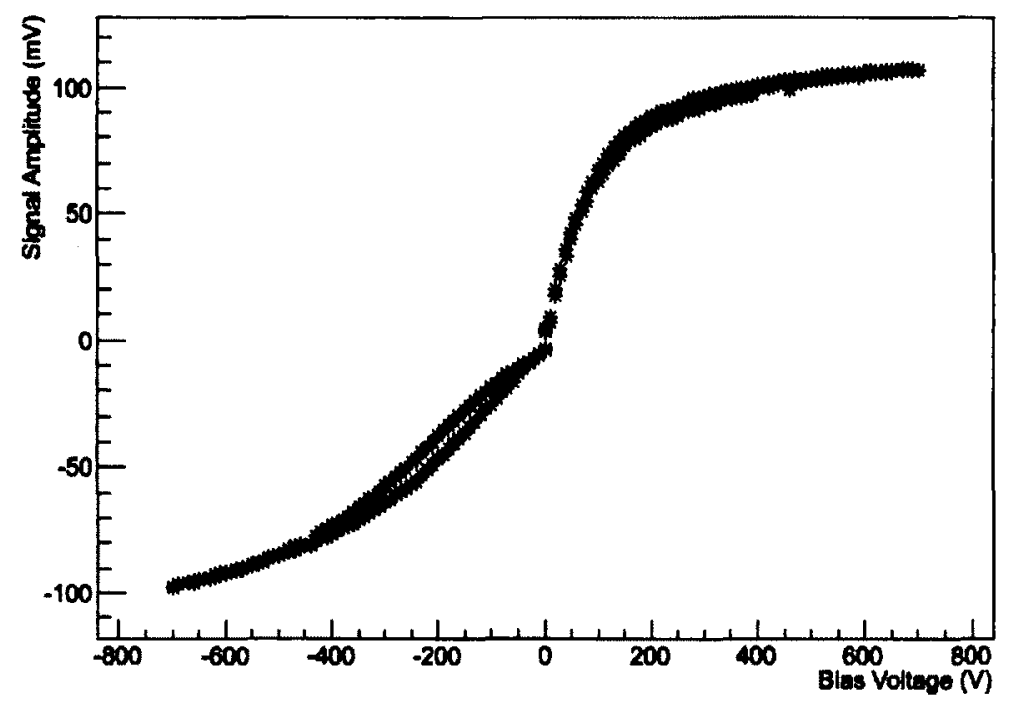

Figure 4.7: Hysteresis data for diamond detector B. The diamond detector was kept in light-proof conditions and pumped with the ${ }^{90} \mathrm{Sr}$ source for 72 hours prior to the test. The bias voltage is changed in increments of $10 \mathrm{~V}$. Data taking is delayed 16 minutes between each voltage increment to ensure that polarization effects are avoided.

test.

To examine detector B's response to different bias polarities, a hysteresis curve was taken. The applied bias voltage is varied in steps and the average signal response to a ${ }^{90} \mathrm{Sr}$ source is measured. An average of 25 waveforms was taken in steps of $10 \mathrm{mV}$ and the peak to peak voltage of the averaged waveform was measured. The voltage was first increased from zero to the detector's optimal bias voltage, in this case 700 $\mathrm{V}$, and then decreased back to zero. The test is then repeated with a negative bias voltage. An ideal detector will show no difference between increasing and decreasing bias voltage or between bias polarities. The results for detector B are shown in Figure 4.7. Only slight differences are observed between increasing and decreasing voltages. However, the voltage required to reach the plateau region for the negative 
bias polarity is noticeably larger. A positive bias polarity was chosen for initial use during the spatial uniformity test.

\subsection{Detector for Spatial Measurements}

Diamond detectors as purchased from DDL are uniformly metalized on both sides; charge deposited by an incident particle is collected throughout the entire volume of the detector, with no indication of the particle's position. For the spatial uniformity test, a position sensitive sensor is required to measure the position of particles that pass through the diamond. For this test, a silicon pixel sensor that had been developed for the ATLAS pixel detectors was chosen. It consists of a silicon sensor bump-bonded to the ATLAS FE-I3 readout chip [56] which is readout by a custom interface, the USBPix readout stand [57].

\subsubsection{FE-I3 Readout Electronics}

The FE-I3 chip is the current ATLAS pixel readout system used in the ID, shown in Figure 4.8 along with the USBpix readout stand. It was developed by the ATLAS collaboration for use in the ATLAS tracking system. To accommodate the various sensor R\&D groups within ATLAS, the chip was designed to operate with any of the proposed sensor materials. This allowed all groups to test sensor materials using the same electronics and software.

FE-I3 single-chip modules are designed to read out sensors with 2880 channels

divided into 160 rows and 18 columns, hereafter referred to as pixels. The majority of pixels are $50 \mu \mathrm{m}$ by $400 \mu \mathrm{m}$. The pixels in the edge columns are slightly larger, with 


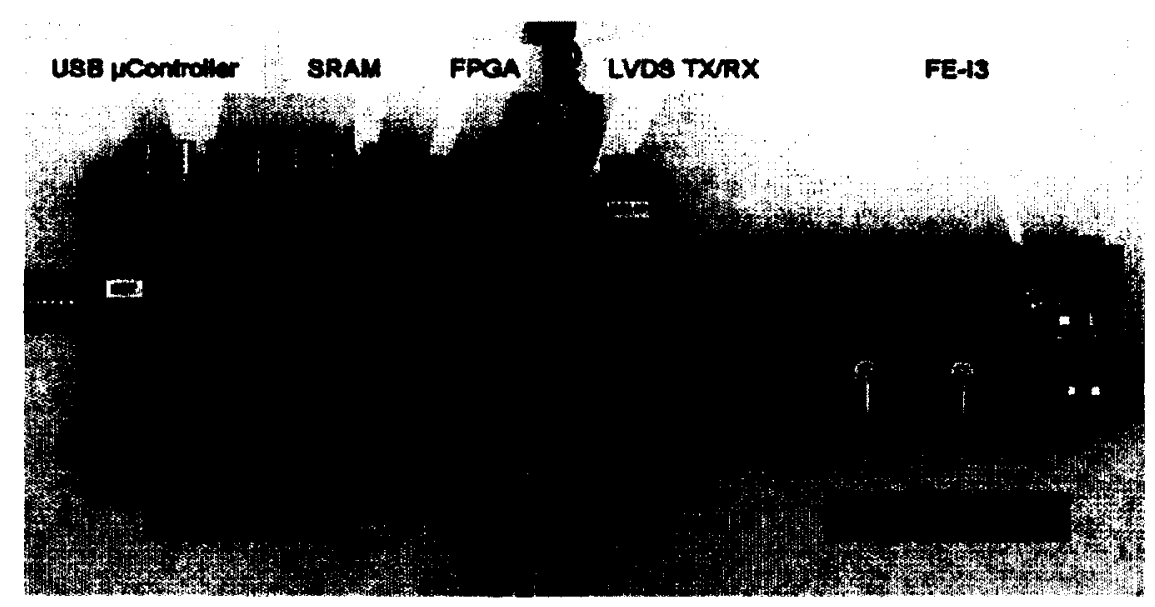

Figure 4.8: USBpix readout system. The ATLAS FE-13 readout chip is shown on the right, bump-bonded to a silicon sensor. The sensor's response to radiation is measured by the FE-13 chip and interpreted by the USBpix readout stand, shown on the left, before being transferred to a DAQ computer via a USB connection, shown on the far left. [57]

a length of $600 \mu \mathrm{m}$. Each pixel has its own charge sensitive amplifier, discriminator, and digital output interface.

Charge gathered in a pixel is amplified by the charge sensitive amplifier and compared against a programmable threshold using the discriminator. The amount of time the signal spends above threshold is known as the Time over Threshold (ToT) and is measured in units of Bunch Crossing ID (BCID). Each BCID is separated by $25 \mathrm{~ns}$ which is the nominal delay between bunch crossings in the ATLAS detector. Since the charge scaling from the amplifier and the discriminator threshold are known through calibration, charge deposited in the sensor can be directly correlated to the ToT count. 


\subsubsection{USBpix}

The USBpix is a readout system developed by the Universität Bonn in Bonn, Germany. It consists of two interlocking boards known as the Multi-IO board and the module adapter card. It has been used to test various sensor technologies prior to mass-production for use in ATLAS.

A USB micro-controller chip on the Multi-IO board takes commands from a computer and converts them into commands to be implemented by the FPGA. The FE-I3 single-chip card is connected to the readout stand via the module adapter card.

The Multi-IO board is connected to a PC via USB and is controlled by a modified version of STcontrol software. This software is under development, and currently lacks the functionality necessary for use in conjunction with other detectors. The software was modified by the Carleton group for the purpose of this experiment, adding functionality that allowed it to be used in conjunction with a diamond detector readout system.

\subsection{Data Acquisition System}

The data acquisition (DAQ) system used during the spatial uniformity test was a modified version of the STcontrol software used by the USBpix readout system. STcontrol records run data in a series of scans of arbitrary length. Data can be gathered based on number of hits, triggers, or end of event words, which occur for every bunch crossing. After reaching the desired scan length, say 4000 hits, STcontrol will either start the next scan or end the data run, depending on whether or not it has reached the last desired scan. 


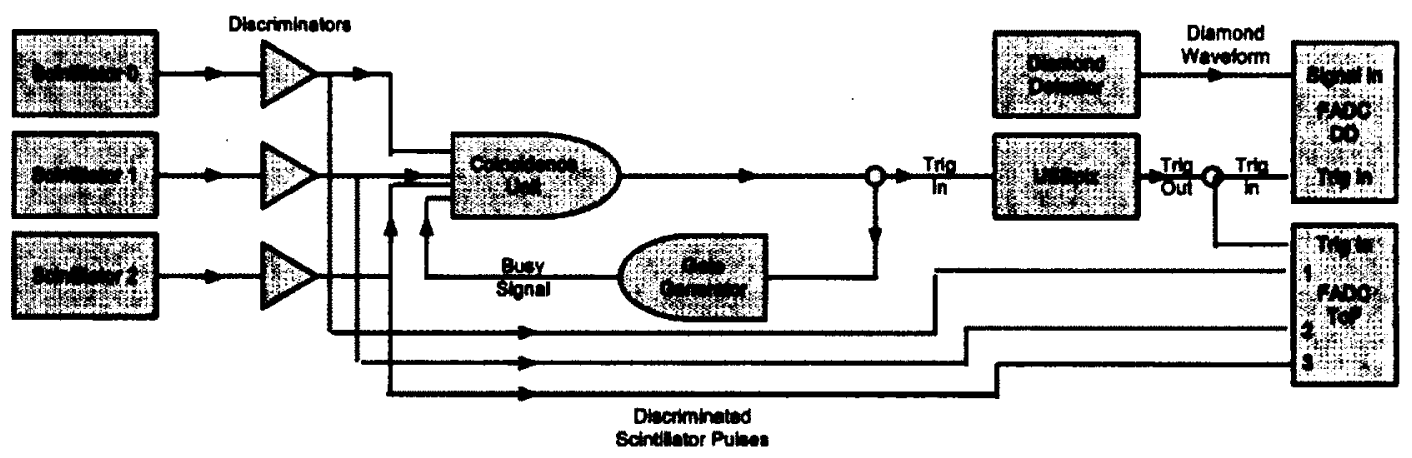

Figure 4.9: Circuit diagram for spatial uniformity test. The scintillator signals are discriminated and sent to a coincidence unit, which is used to trigger the USBpix readout stand. The USBpix then triggers the FADC modules. A busy signal is sent to the coincidence unit during the USBpix's processing time (approximately $7 \mu s$ ) to prevent additional triggers.

For every trigger received, the USBpix examines all 2880 pixels for hits across a set number of BCIDs. The number of bunch crossings examined was set to 16 , for a time window of $400 \mathrm{~ns}$. The USBpix SRAM fills at approximately 24,000 triggers, forcing the readout of trigger data. To maximize the live time of the experiment (the amount of time spent gathering data as a fraction of the total time), the number of triggers per scan was set to 20,000 . At the end of every scan the USBpix outputs a file containing the data for every trigger. This file contains the hit position and ToT for every triggered pixel at every BCID.

Two VME Flash Analog-to-Digital Converter (FADC) modules $^{4}$ were used to digitize the signals produced by the diamond detector and the scintillator triggers. Each module was capable of digitizing four channels simultaneously at a rate of $1 \mathrm{MHz}$ - $500 \mathrm{MHz}$ (for a $2 \mathrm{~ns}-1 \mu$ s period between samples) per channel. Up to $128 \times 10^{6}$ samples per channel could be stored in memory, for a time window of $0.256 \mathrm{~s}-128$ s. The modules have a pre/post-trigger capability, which allows them to output a

\footnotetext{
${ }^{4}$ Struck SIS3350
} 
specific time window from any point in their memory.

The FADC modules continuously store the signals they digitize in buffers. When a trigger is received, the FADC outputs the information stored in a chosen time window at an arbitrary time either before or after the trigger signal. Early laboratory tests showed that the number of triggers received by the FADC modules and the USBpix quickly desynchronized if the trigger was sent to them directly. For this reason the trigger was instead sent to the USBpix first, which produced an output signal for every trigger it received. This output signal was used to trigger the FADC modules. The USBpix took $6.3 \mu$ s to process events and produce an output signal. The FADC used to record the diamond detector signal was calibrated to record a $2 \mu \mathrm{s}$ time window with a $10 \mathrm{~ns}$ sampling period $7 \mu$ s prior to when it received the trigger, as the diamond signal could not be effectively delayed. The original trigger signal was also recorded by this FADC. The scintillator signals were delayed by $6.3 \mu \mathrm{s}$ using the trigger logic and a $1.008 \mu$ s time window with a 2 ns sampling period was recorded, with the intention of measuring the time of flight (ToF) of triggering particles. A veto gate was used to prevent additional triggers during this time window.

The FADC modules were read out via a USB connection from the VME crate to the DAQ computer. Modifications were made to STcontrol to allow operation of the FADC modules. A block diagram of the trigger logic used during the spatial uniformity test is shown in Figure 4.9.

The FADC data are recorded in two separate files at the end of every scan, one for the diamond and trigger data, the other for the ToF data. These two data files are later merged with the USBpix output to allow for simple data manipulation. Cosmic rays were used to test the DAQ in the laboratory prior to the spatial uniformity test, since the ${ }^{90} \mathrm{Sr}$ source could not penetrate the two detectors and the scintillator 


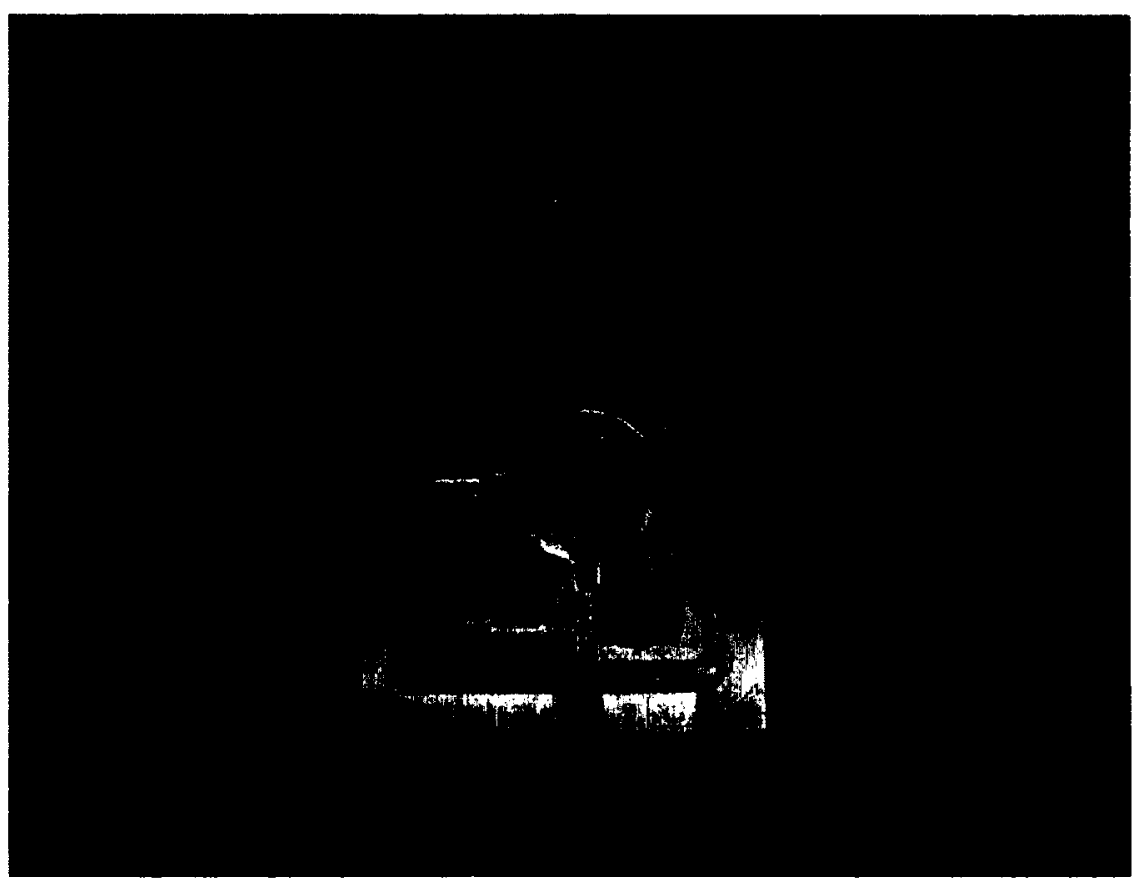

Figure 4.10: Custom holder designed for use in spatial uniformity test. The FE-13 chip is inserted from below and held in place. The holder makes use of two small pins to hold the diamond detector in place while allowing it to rotate $90^{\circ}$ easily.

triggers. Initial problems were worked out in the lab prior to shipping the equipment to TRIUMF for the spatial uniformity test.

\subsection{Diamond Spatial Uniformity Test}

The two detectors were placed $3 \mathrm{~mm}$ apart, which minimized the amount of scattering between detectors, and housed within an aluminum case which provided the electrical and light shielding necessary for the detectors to operate. The detector holder is shown in Figure 4.10. Due to the rectangular shape ( $50 \mu \mathrm{m} \times 400 \mu \mathrm{m})$ of the USBpix pixels and the lack of additional detectors with which to form a true beam telescope, the position resolution along one axis is an order of magnitude worse than the other. 


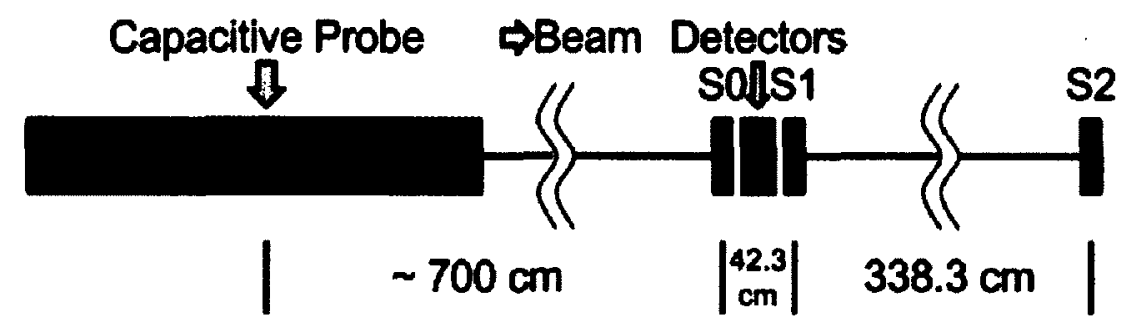

Figure 4.11: Diagram of components exposed to particle beam during spatial uniformity test.

In an attempt to mitigate this problem, the diamond detector holder was designed such that the detector could be rotated $90^{\circ}$ with respect to the USBpix silicon sensor, with the intention of combining multiple data sets and improving the position resolution. The centres of the two detectors required aligning in order to maximize the amount of overlapping surface area for both orientations. This alignment was accomplished using an optical microscope $e^{5}$. The centres of the two detectors were aligned to within $10 \pm 51 \mu \mathrm{m}$ of each other. The large error is due to a backlash error inherent in the microscope. After the alignment, aluminum foil was placed between the two detectors to provide additional electrical shielding to the diamond detector, reducing the amount of electronic noise induced in the diamond by the operation of the FE-I3 readout chip.

The spatial uniformity test took place in September of 2011 in TRIUMF's M11 beamline. M11 is a secondary beamline meant to produce a large number of pions. However, due to a broken septum magnet, the primary proton beam no longer collides with the designated beam target. This results in the production of a low numbers of electrons, muons, and pions. Due to the limitation of our DAQ, a low flux was preferable, making M11 perfectly suited to our task.

\footnotetext{
${ }^{5}$ Fratelli Koristka Milano R5
} 


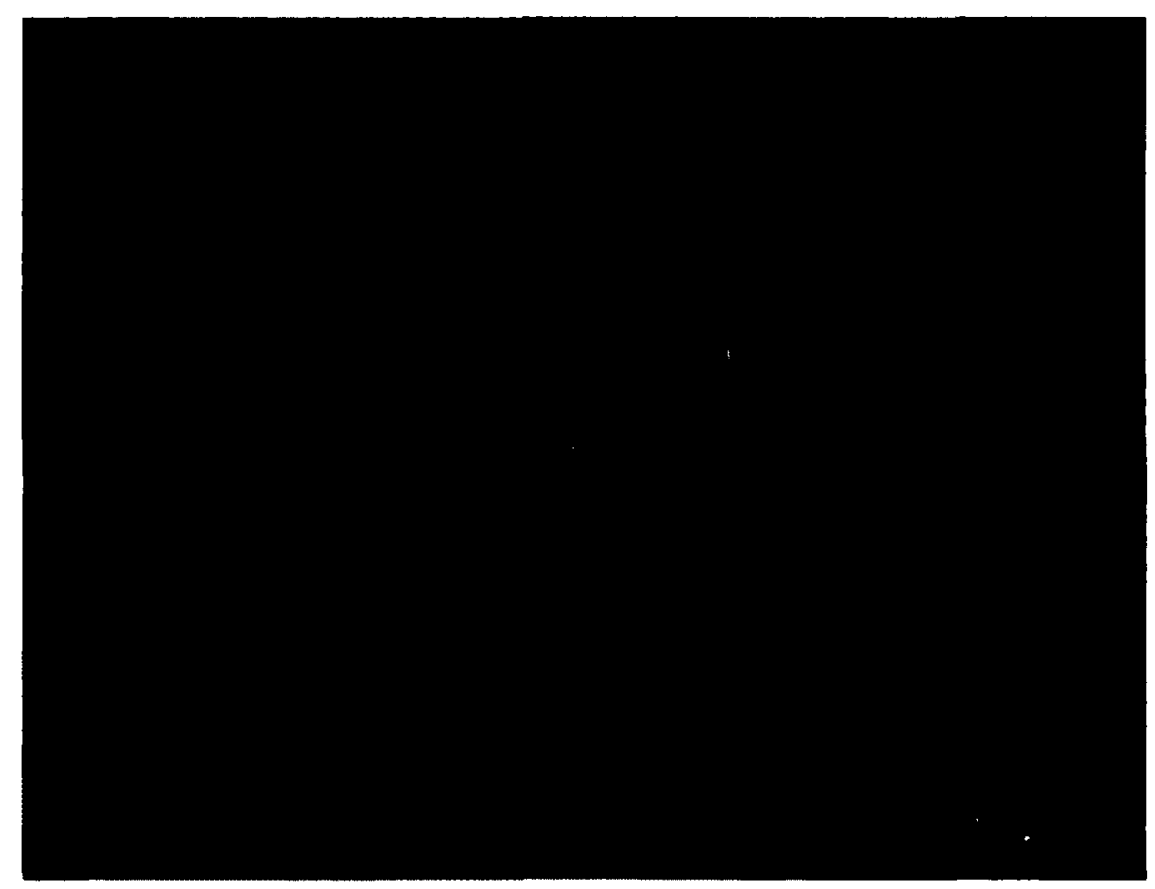

Figure 4.12: Placement of scintillators in M11 beam area. The end of the beam line can be seen in the top left along with the locations of scintillators 0 and 1. The scaffolding used to hold the detector housing in place is shown on the bottom.

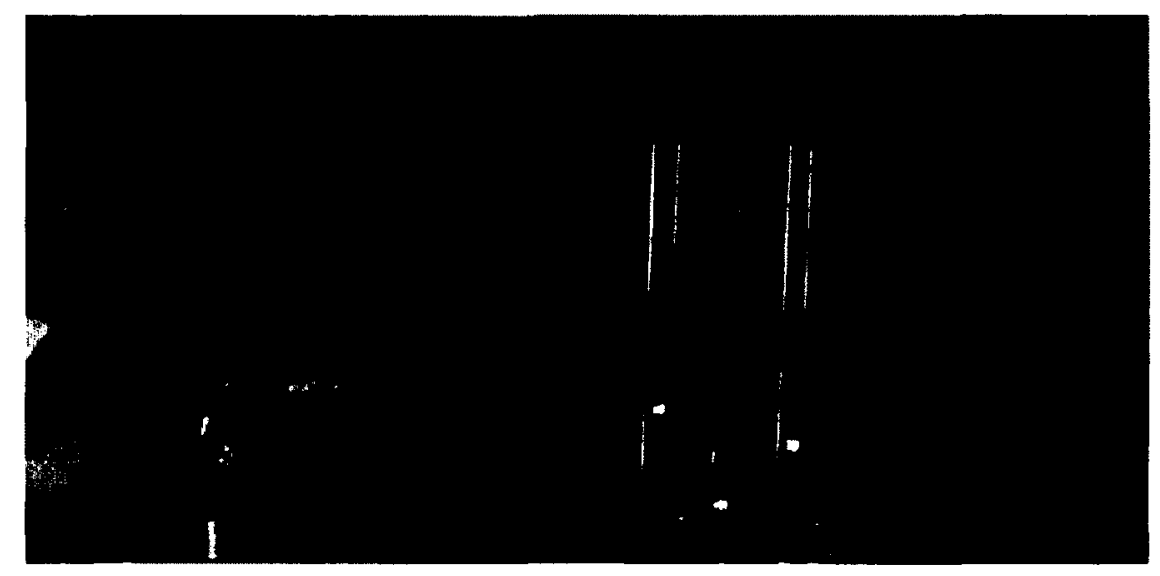

Figure 4.13: Placement of detectors in M11 beam area with respect to the beam line. The detector housing is located on the opposite side of the scaffolding. 


\begin{tabular}{lll}
\hline \hline Scintillator & Dimensions & Placement \\
\hline 0 & $2 \times 2.5 \mathrm{~cm}^{2}$ & $14 \mathrm{~cm}$ \\
1 & $4 \times 5 \mathrm{~cm}^{2}$ & $56.3 \mathrm{~cm}$ \\
2 & $15 \times 15 \mathrm{~cm}^{2}$ & $394.6 \mathrm{~cm}$ \\
\hline \hline
\end{tabular}

Table 4.2: Scintillator dimensions. Placement is measured from the end of the beam pipe.

A simple diagram of the beam area during the spatial uniformity test can be found in Figure 4.11. Figures 4.12 and 4.13 show the placement of the detectors and scintillators 0 and 1 with respect to the end of the beam line. The detectors were placed approximately $15 \mathrm{~cm}$ from the end of the beam pipe; since the angular distribution of the beam is unknown, there was no optimal detector alignment with respect to the beam. The detector housing had been notched to denote the centre of the detectors. These markings were used to align the detectors with the centre of the beam, which was very broad; the beam profile had a sigma of $8 \mathrm{~cm} \times 2 \mathrm{~cm}$ due to the broken septum magnet.

One scintillator was placed in front of the detectors at a distance of approximately $1 \mathrm{~cm}$ while another was placed approximately $41 \mathrm{~cm}$ downstream of the detectors. A much larger scintillator was placed as far downstream as possible, with the intention of measuring the ToF of particles and to collimate the beam. The placement and dimensions of the scintillators can be found in Table 4.2. A coincidence signal from the scintillators was used to trigger the detectors. Initially, only scintillators 0 and 1 were used for trigger purposes. It was found that only $20 \%$ of particles passed through scintillator 2, making ToF measurements impossible on the majority of events. Scintillator 2 was added to the trigger logic to ensure that ToF measurements could be made during the analysis. 
The amplifier for the diamond detector was located approximately $15 \mathrm{~cm}$ from the detector housing. To reduce noise levels in the amplifier, it was connected to the diamond via a $30 \mathrm{~cm}$ low capacitance cable ${ }^{6}$. All signal outputs and voltage supplies were connected via $30 \mathrm{ft}$ cables to a monitoring platform above the M11 area where the DAQ computer was located. The DAQ system was monitored remotely using a VNC client due to high neutron levels on the platform.

The DAQ system recorded a wide variety of data, including the USBpix hit position, ToT, and BCID of each event. The signals produced by the diamond detector, the original trigger waveform, and scintillators 0 and 2 were recorded for each event via FADC modules. The signal from the beamline's capacitive probe was also recorded by the FADC with the intentions of making ToF measurements. However, the signal was unstable and proved to be unusable. The voltage supplies for both detectors were monitored for fluctuations in output via GPIB cables and custom monitoring software. The high voltage (HV) supplies remained stable throughout the test, as did the leakage current produced by the detectors. The Cremat amplifier and the USBpix electronics required several low voltage (LV) supplies, which were also monitored. The LV supplies' voltage and current remained stable throughout the test.

Three NIM counting modules were used to monitor the trigger rate and estimate the live time of the experiment. The counters measured the number of scintillator coincidences with and without the $7 \mu$ s veto signal ( $\mathrm{C} 2$ and $\mathrm{C} 1$ respectively) and the number of output signals produced by the USBpix (C3). The difference between counters $\mathrm{C} 1$ and $\mathrm{C} 2$ determines the number of events discarded due to the delay gate necessary to prevent multiple hit events. Only $7 \%$ of events throughout the test were

\footnotetext{
${ }^{6} \mathrm{RG}-62 / \mathrm{U}, 13.5 \mathrm{pF} / \mathrm{ft}$
} 
discarded in this fashion. $\mathrm{C} 3$ as a fraction of $\mathrm{C} 2$ provides an estimate of the test's live time, which was determined to be $63 \%$. 


\section{Chapter 5}

\section{pCVD Diamond Spatial \\ Uniformity Measurements}

Spatial uniformity measurements of a pCVD diamond detector took place at TRIUMF in September of 2011. The detector was exposed to a low-flux beam of electrons, muons, and pions over the course of 10 days. The diamond detector's signal response was recorded for each impinging particle and mapped to a $50 \mu \mathrm{m} \times 400 \mu \mathrm{m}$ pixel position in a secondary detector. The original plan for the spatial uniformity test was to gather only 2 sets of data; one for each of two orientations of the diamond. The alignment for these two orientations can be seen in Figure 5.1, where the diamond is rotated by $90^{\circ}$.

Additional data sets were gathered due to the unexpected response of the diamond detector to a positive applied voltage. Although the diamond detector had shown a larger signal response using a positive bias voltage in all previous tests, during the spatial uniformity test initially little or no signal was observed for a positive bias voltage. Applying a negative bias voltage produced the expected signal amplitude. As 


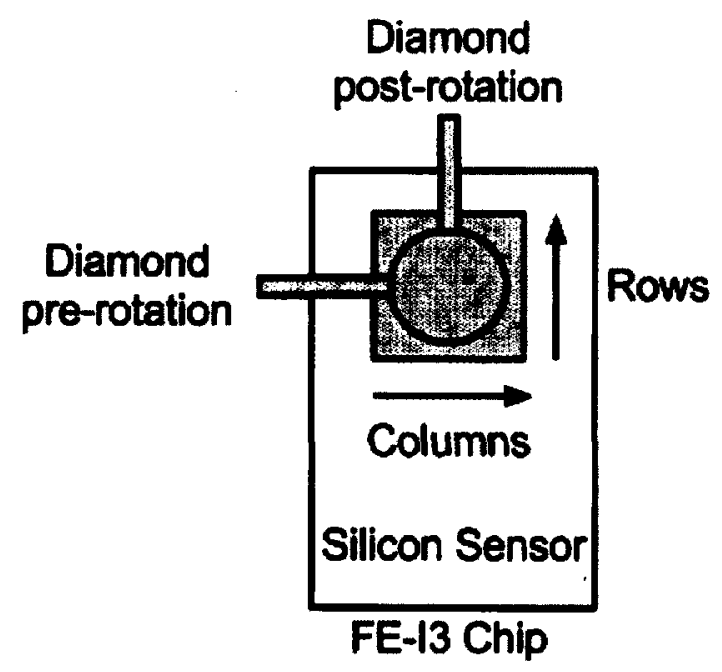

Figure 5.1: Orientation of diamond detector pre and post rotation with respect to the USBpix. The columns and rows refer to the axes depicted on the USBpix hitmaps, as shown in Figures 5.4 and similar figures.

such, a negative bias polarity was used for most of the test. In the end, seven sets of data were gathered, varying in beam polarity (particles delivered were either positive or negative), bias polarity (bias supplied to diamond was either positive or negative at $700 \mathrm{~V}$ ), the number of scintillator triggers used and the diamond alignment. A list of the data sets taken along with the conditions for each data set can be found in Table 5.1.

This chapter will describe the results of the spatial uniformity test. Most of the chapter is devoted to the analysis of the diamond detector results, including data calibration, checks on data quality, comparisons of data sets, and the results of the spatial uniformity test. 


\begin{tabular}{|c|r|c|c|c|c|}
\hline Data Set & \# Events & Bias Polarity & Beam Polarity & $\begin{array}{c}\text { \# Scintillator } \\
\text { Triggers }\end{array}$ & Rotation \\
\hline 1 & 782539 & - & + & 2 & Pre \\
\hline 2 & 1279416 & - & + & 3 & Pre \\
\hline 3 & 1131123 & - & + & 3 & Post \\
\hline 4 & 893786 & + & + & 3 & Post \\
\hline 5 & 302024 & + & - & 2 & Post \\
\hline 6 & 375565 & - & - & 2 & Post \\
\hline 7 & 573207 & - & + & 3 & Post \\
\hline
\end{tabular}

Table 5.1: Parameters for each data set and the number of events in each (after the cuts described in Section 5.1). The data sets are assigned a number, and will hereafter be referred to by this number. 


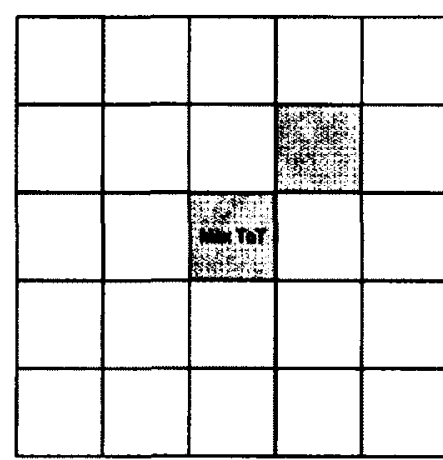

(a)

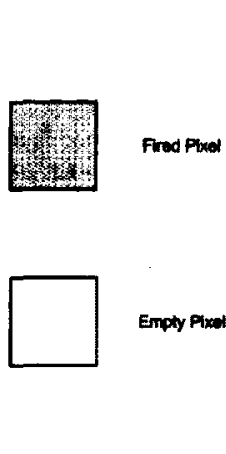

noty Pixed

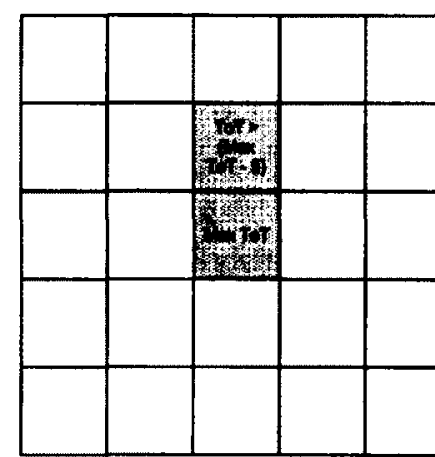

(b)

Figure 5.2: Diagram of possible events in the USBpix silicon sensor. Figure a) shows an event in which non-adjacent pixels fire. These events are removed by the first cut. Figure b) shows an event where an adjacent pixel has registered a ToT similar to the maximum ToT of the event. These events are removed by the second cut.

\subsection{USBpix and Scintillator Analysis}

The USBpix detector was used to determine the hit position of particles passing through the diamond detector. The USBpix also produced a measure of charge deposition known as Time over Threshold (ToT). The USBpix used was calibrated to use a charge threshold of 4000 electrons for ToT measurements and to produce an average ToT of $30 \mathrm{BCID}$ for a MIP. Any number of pixels may fire (have non-zero ToT) during an event, due to both charge sharing between pixels and random noise. To determine the position of the incident particle, two cuts were applied. First, the pixel with the maximum ToT for a given event was found. If pixels that were not directly adjacent to this pixel (secondary pixels) fired as well then the event was discarded; the additional pixels are either due to random noise fluctuations or a multiple particle event. Note that diagonals are not considered to be adjacent. If any pixel adjacent 
to the pixel with the maximum ToT has a ToT that is similar to the maximum ToT then the event is also discarded, as this could also indicate a multiple particle event. A simple diagram of these events is shown in Figures 5.2. Of the 20,000 triggers received per scan, approximately 2000 triggers correspond to events that meet these criteria. The selected events are used to produce the results presented in this chapter, except where noted otherwise.

Figures 5.3 and 5.4 show the cell occupancy of the USBpix detector for data set 2. The circular shape is likely due to either the beam profile or to beam particle selection by the scintillators. The results are similar for all data sets. Figure 5.5 shows example histograms of the ToT registered by the USBpix over the course of an entire data set. Figure 5.5a shows the ToT for all triggered pixels, prior to the application of the two cuts described earlier. It shows the characteristic double peak expected from the firing of secondary pixels. Figure 5.5b shows the maximum ToT of events after the cuts have been applied. It peaks at a ToT of 30 , as calibrated for a MIP. The results are similar for all data sets, though the negative particle data does show a slightly lower ToT.

Three scintillators were used for trigger purposes during the test. There was insufficient time prior to the spatial uniformity test to include a high resolution timeto-digital converter (TDC) module in the DAQ system for ToF measurements. Instead, scintillators 0 and 2 (Refer to Section 4.4), as well as the beam line's capacitive probe, were digitized using a FADC. The capacitive probe's response proved too unstable to be useful. A histogram of the ToF between scintillators 0 and 2 can be found in Figure 5.6. No particle differentiation was possible given the resolution of the available FADC. The results of the diamond amplitude measurements could therefore not be sorted by particle type and compared. Had the capacitive probe signal been stable 


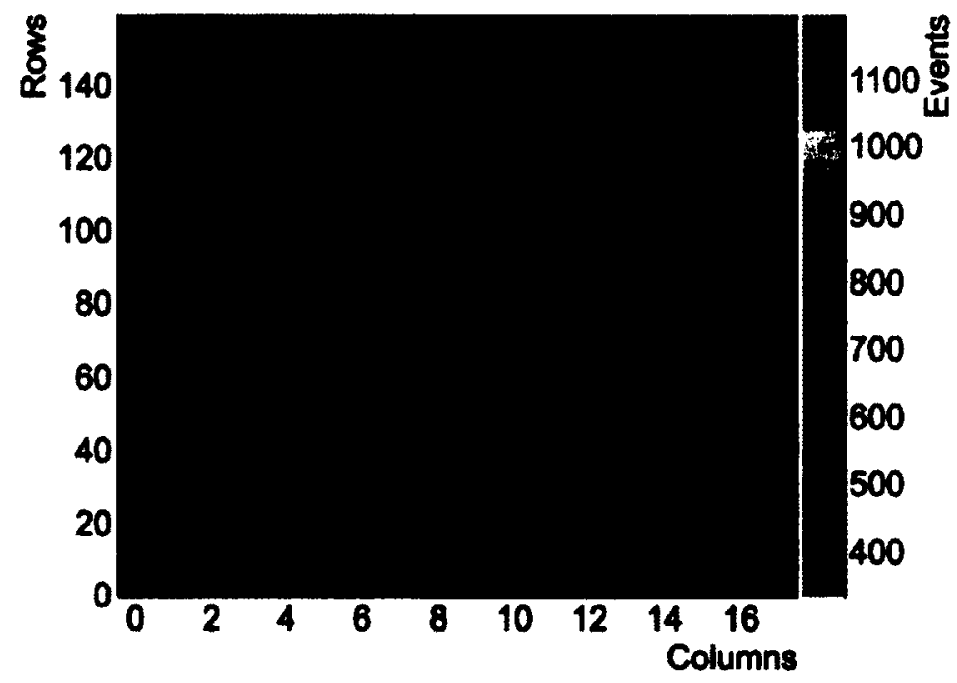

Figure 5.3: Pixel occupancy for the USBpix detector for data set 2. The edge columns and rows fire up to three times more often than the pixels in the centre, making observation of fine detail in the centre pixels difficult.

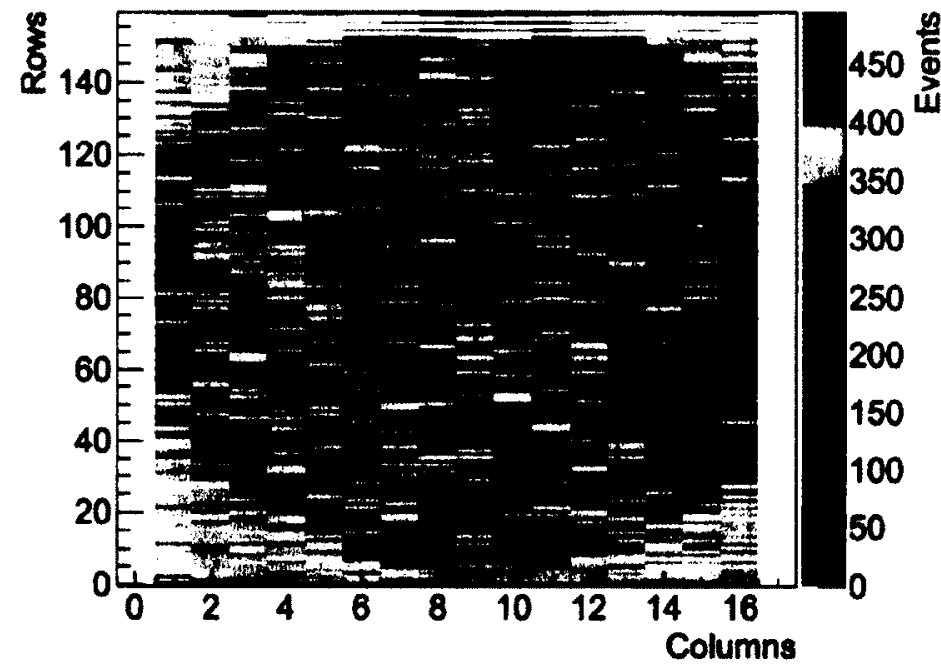

Figure 5.4: Pixel occupancy for the USBpix detector for data set 2. The edge columns and rows have been masked. This plot shows that the illumination of the pixel detector by the beam was uniform with some decrease at the corners, probably caused by the beam profile or the effect of the scintillator triggers. 


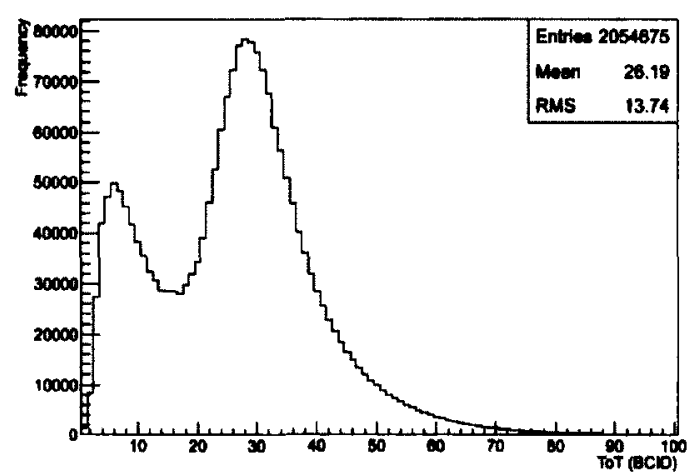

(a)

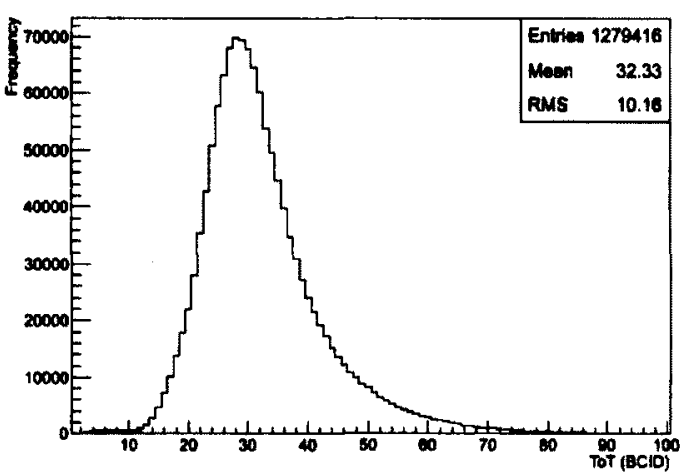

(b)

Figure 5.5: ToT data produced by the USBpix for data set 2. a) is the ToT for all triggered pixels. b) is the maximum ToT for events after cuts have been applied.

enough for use it is likely that the FADC resolution would have sufficed.

\subsection{Diamond Analysis}

Examples of single waveforms from the diamond detector are given in Figure 5.7. High levels of noise make single waveform measurements difficult. Averaging of waveforms was required to remove random noise, although a structured background remained, as shown in Figure 5.8. The structured background noise was caused by electronic pick up from the USBpix readout. This background noise was isolated in each data set by averaging the pulse shape over the signal regions where the USBpix and the diamond detector did not overlap. Theoretically, this region, which is shown in Figure 5.9, should contain no signal whatsoever, allowing for direct background measurement. Since the centre of the diamond detector was not perfectly aligned with the centre of the USBpix silicon sensor, a simple radial cut could not be applied. Instead, pixels were chosen by placing a cut on the peak to peak amplitude of the average pixel 


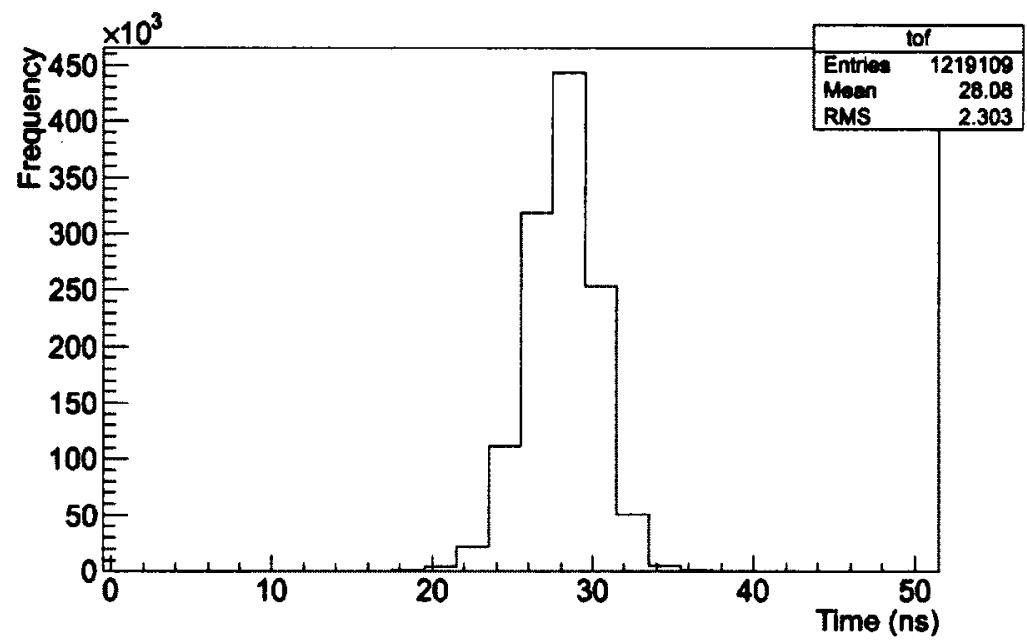

Figure 5.6: Histogram of the time difference measured between scintillators 0 and 2 for data set 2. Note that the offset of both scintillator signals is unknown.

waveform within the signal region. Pixels with an amplitude less than 15(8) ADC values were considered to be background pixels for the negative(positive) bias voltage data sets. Since these amplitudes correspond to the corners of the USBpix, this method was deemed suitable. As shown in Figure 5.10, there are slight differences in the signal background between positive and negative bias polarities, caused by the applied electric field extending into the edge regions of the diamond. This is due to the fact that the metalization used to supply the electric field does not extend onto the corners of the diamond detector, as discussed in Section 4.1. Removal of the background produces a much cleaner signal response, as can be seen in Figure 5.11. The signal pedestal is then calculated using the first 15 points of the averaged waveform and subtracted. In subsequent analysis steps the absolute value of the resulting signal is used to make the analysis and data comparison processes simpler.

Three different techniques were used to extract the signal amplitude from the averaged waveforms, the first being an integral over the signal region, calculated numerically using the ROOT [58] Integral function. The optimal signal integral range 


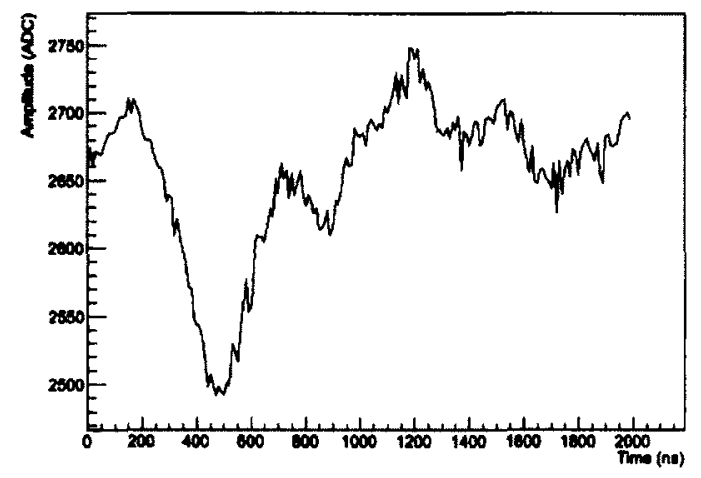

(a) Negative bias voltage applied

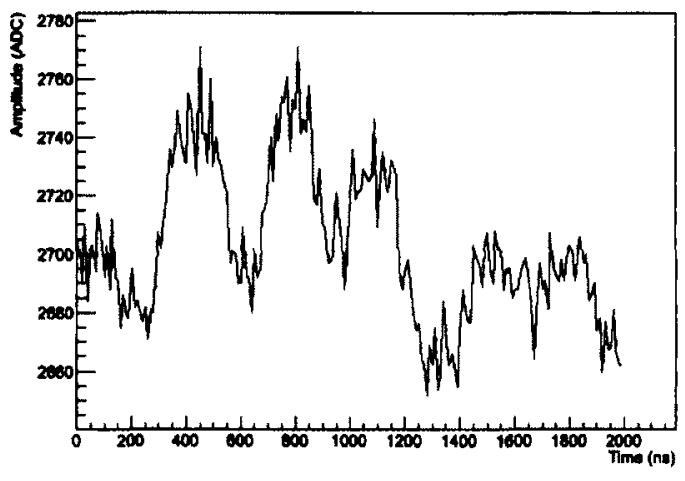

(b) Positive bias voltage applied

Figure 5.7: Sample waveforms from the diamond detector. The background noise is comparable to the signal for positive bias voltages.

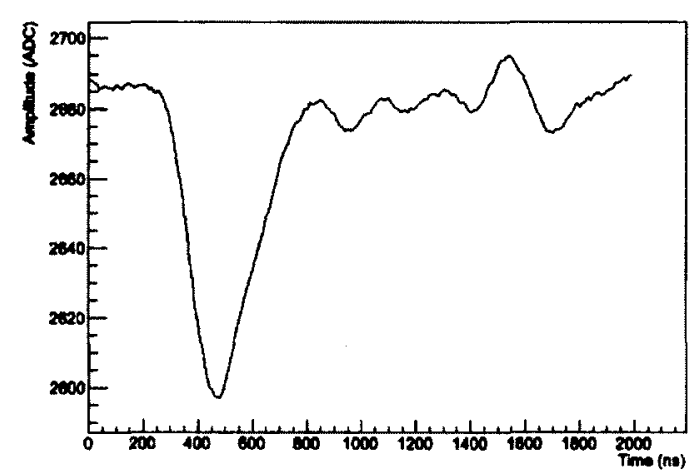

(a) Negative bias voltage applied

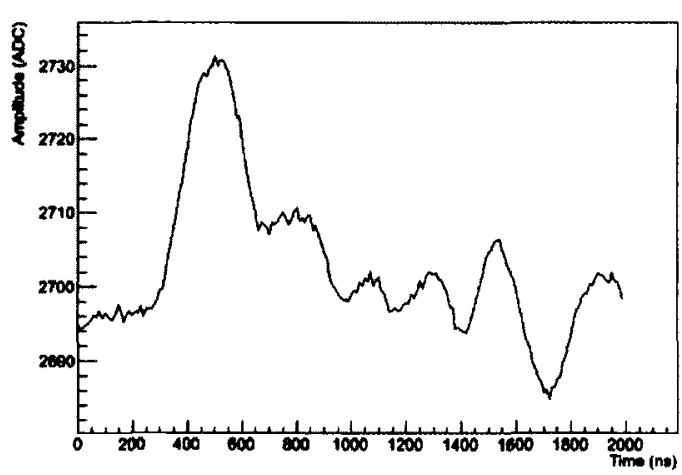

(b) Positive bias voltage applied

Figure 5.8: The signal waveforms from all events in a pixel for an entire data set are averaged to create a cleaner signal. Signal from the positive bias voltage is now clearly visible. 


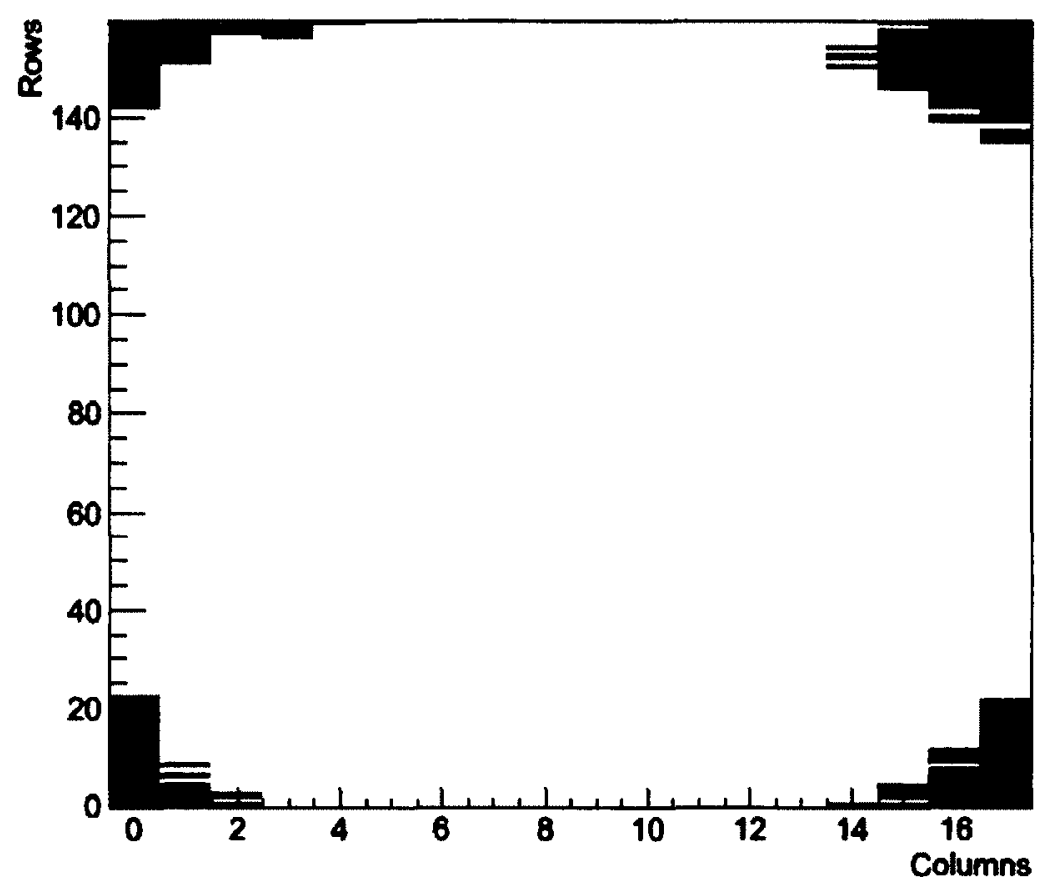

Figure 5.9: Location of pixels used to measure electronic background in data set 2.

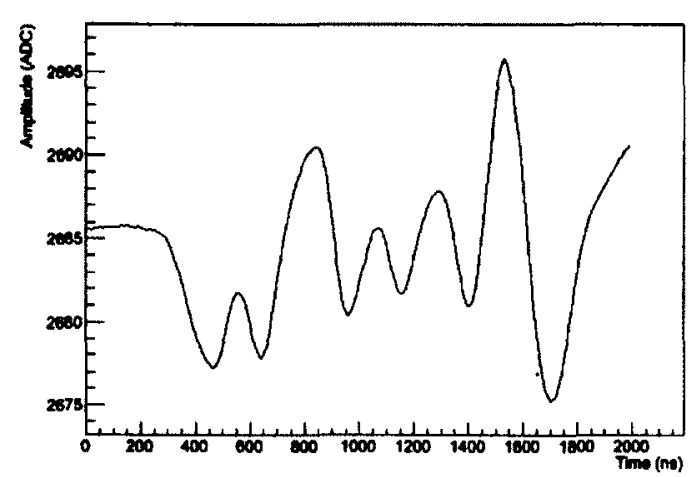

(a) Negative bias voltage applied

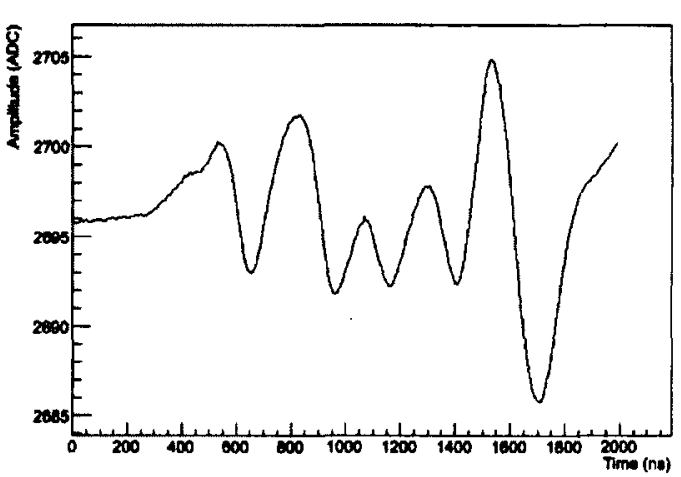

(b) Positive bias voltage applied

Figure 5.10: Averaged signal background. The backgrounds differ slightly due to the non-uniform electric field at the edge of the diamond detector. 


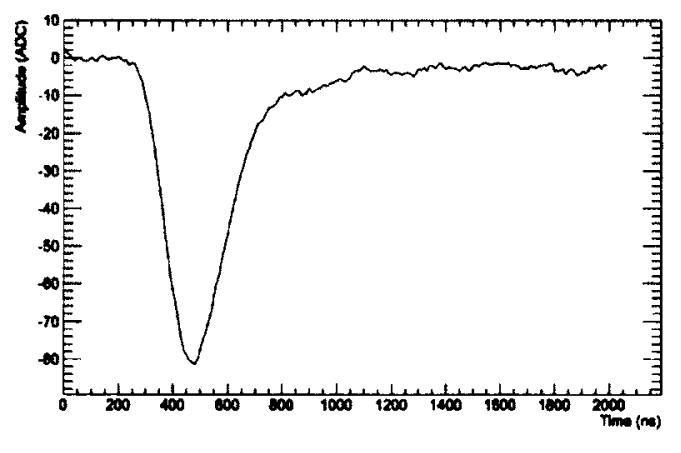

(a) Negative bias voltage applied

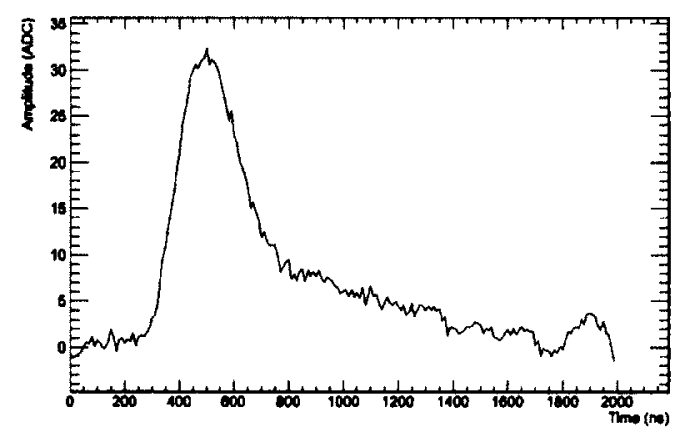

(b) Positive bias voltage applied

Figure 5.11: Averaged signal waveform after the removal of signal background. The resulting waveforms allow the signal amplitude to be accurately obtained.

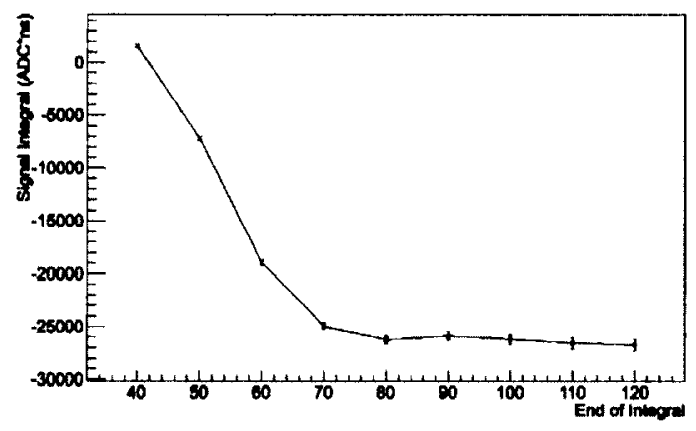

(a) Signal integral amplitude

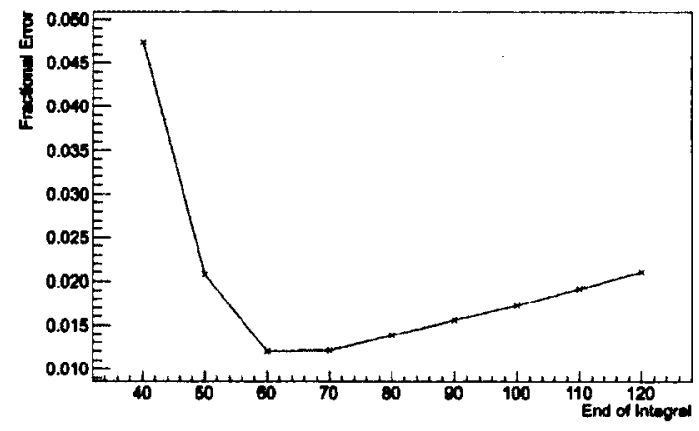

(b) Signal integral fractional error

Figure 5.12: Signal integral range tests. All integrals begin at point 20 (or $200 \mathrm{~ns}$ ) and end at the point stated on the $x$-axis. The fractional error is simply the absolute value of the uncertainty on the integral as a fraction of the integral. 
was determined by integrating from point 20 (or $200 \mathrm{~ns}$ ) of the waveforms to varying end points and comparing the resulting integrals and their fractional errors, shown in Figures 5.12. The fractional error is at its lowest for an end point of 60 (600 ns) and rises slowly at higher values. The integral plateaus at point 80 (800 ns), and based on this, point 80 was chosen as the end point for the integration. This measure is complicated by the fact that the peak position of the diamond waveform varies over data sets due to changes in the trigger logic. To compensate, the integration range must also be varied. To keep the same relative integration range, the signal region is always considered to extend from 21 points prior to the peak position to 39 points after for the purpose of calculating the signal integral.

The second measure of the signal amplitude was of the peak value within the signal region, termed the peak amplitude. Finally, the third method was to fit the averaged waveform in each pixel to the averaged waveform for the entire data set (after the removal of the background signal and pedestal from both). An example of fitted data is shown in Figure 5.13.

\subsubsection{Calibration of Diamond Detector Signal}

The FADC module outputs the diamond signal for each time bin in ADC values. To measure the charge collection distance of the diamond detector, $\mathrm{ADC}$ values must be converted into an amount of charge collected, allowing for comparisons between the results presented here and those published in the literature. To accomplish this, a calibration signal was fed into the test input of the preamplifier. The test input is connected to a capacitor that converts an input voltage into an equivalent charge input. By varying the input pulse amplitude from a pulse generator ${ }^{1}$, it was possible

\footnotetext{
${ }^{1}$ Agilent 81130A Pulse/Pattern Generator
} 


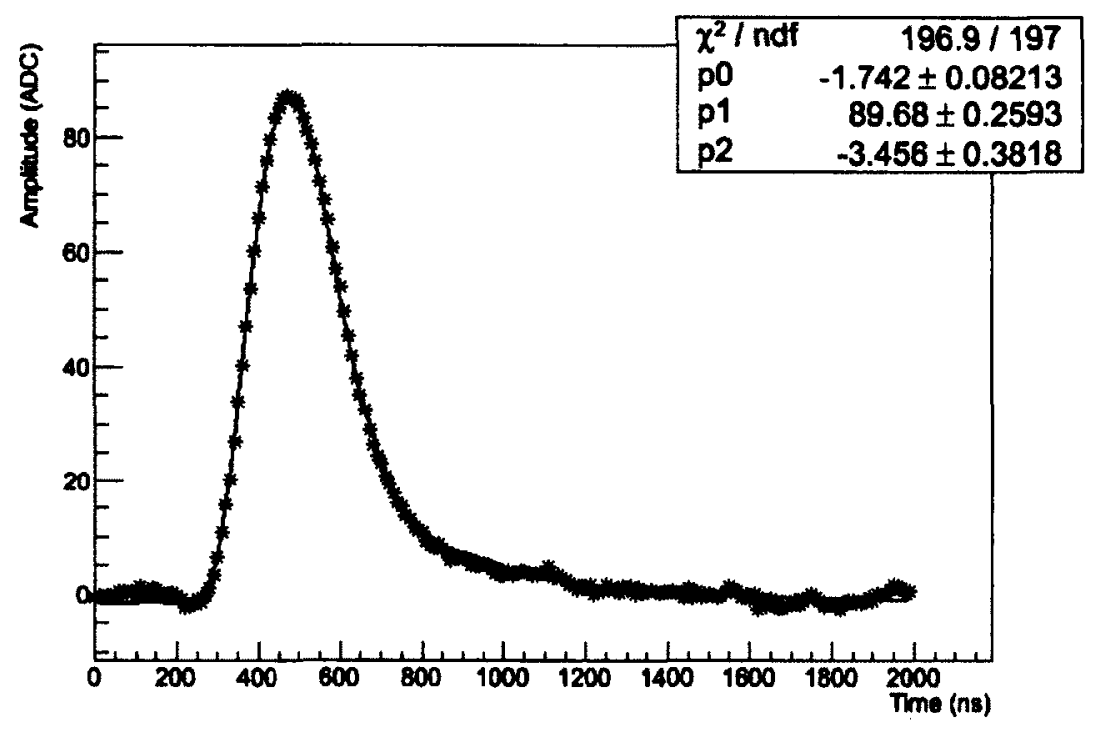

Figure 5.13: Averaged signal from a pixel in data set 2 shown as stars with the fit to the averaged signal from data set 2 shown as the line. Parameters 0,1 , and 2 are the $y$-offset, amplitude, and $x$-offset respectively. 


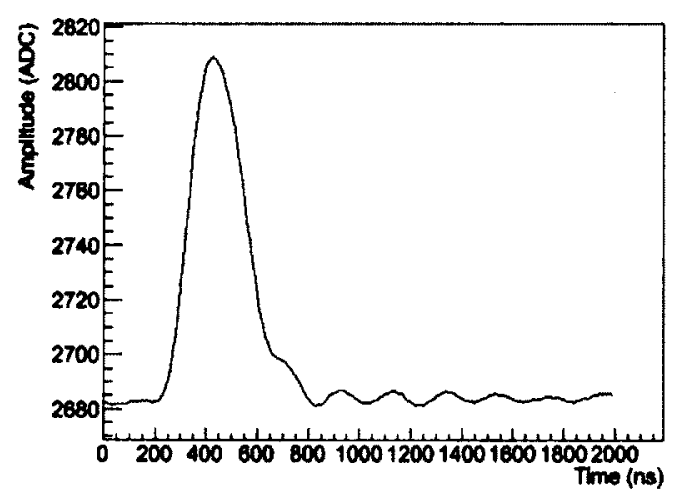

(a)

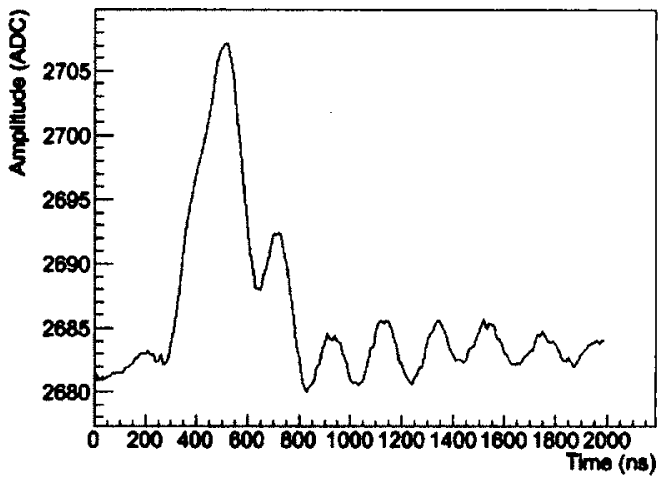

(b)

Figure 5.14: Average of 2000 waiveforms produced by the Cremat amplifier for two pulse generator settings. a) shows the result for the $250 \mathrm{mV}$ pulse generator setting. b) shows the result for the $0 \mathrm{mV}$ setting, which is assumed to be the background noise seen by the amplifier.

to calibrate the signal response of the amplifier used for the diamond detector in terms of the ADC value for a given voltage at the calibration input, which in turn can be converted into an amount of charge on the input.

The minimum output of the pulse generator was $50 \mathrm{mV}$. This value is well above the equivalent voltage produced by the average energy deposited in the diamond detector by a MIP. Several passive attenuators were used to reduce the pulse generator output by a factor of 200 . The amplifier's response was then measured for sets of 2000 waveforms at pulse generator settings ranging from 50 to $250 \mathrm{mV}$, in increments of $20 \mathrm{mV}$. The averaged signal response for the $250 \mathrm{mV}$ pulse generator setting, as well as the averaged background waveform, are shown in Figure 5.14. The background is subtracted from the average waveform prior to further measurements. Note that the background noise seen in the amplifier during calibration differs from the background noise observed during the spatial uniformity test. 


\begin{tabular}{|l|l|l|}
\hline Fit Parameter & Slope & Y-Intercept \\
\hline Integral Calibration & $103.7 \pm 0.7 \mathrm{ADC}^{*} \mathrm{~ns} / \mathrm{mV}$ & - \\
\hline Peak Calibration & $0.434 \pm 0.002 \mathrm{ADC} / \mathrm{mV}$ & - \\
\hline Fit Calibration & $0.4326 \pm 0.0004 \mathrm{ADC} / \mathrm{mV}$ & - \\
\hline Attenuator Calibration & $5.10 \times 10^{-3} \pm 0.02 \times 10^{-3}$ & $0.066 \pm 0.007 \mathrm{mV}$ \\
\hline
\end{tabular}

Table 5.2: Fit parameters for each signal amplitude measurement method and the amplitude of the attenuated output of the pulse generator.

Measurements of the background subtracted signal amplitude using the peak amplitude, signal integral and fit methods were made for the averaged waveform of each set. The signal amplitude versus the input pulse amplitude was plotted and the points fit to a straight line with a y-intercept constrained to zero. The difference between the fit values and the recorded data points was also examined for each measurement type. The results are given in Figures 5.15 - 5.17.

To determine the actual output of the attenuated pulse generator, the output was fed to an oscilloscope $e^{2}$ and its response measured. The low amplitude of the attenuated output combined with the $1 \mathrm{mV} / \mathrm{div}$ limit of the oscilloscope made measuring low amplitude pulse generator settings difficult. As such, the measurements were instead made between 200 and $400 \mathrm{mV}$, in $20 \mathrm{mV}$ increments, and fit to a straight line. The results are given in Figure 5.18. The difference plot at the bottom of Figure 5.18 shows two separate data populations, which differ by 0.01688 $\mathrm{mV}$. This is due to random fluctuations in the least significant bit of the oscilloscope's measurement. The resulting fit parameters for each method are given in Table 5.2.

\footnotetext{
${ }^{2}$ Agilent Technologies Infiniium DSO9404A
} 


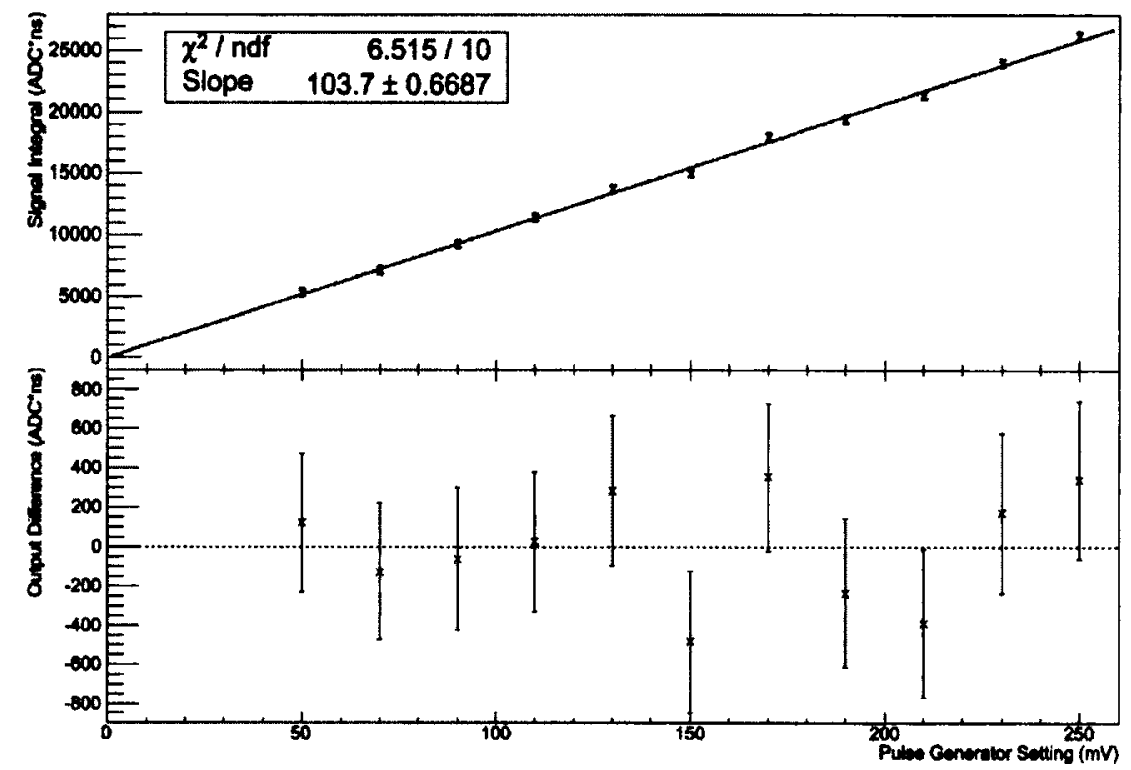

Figure 5.15: Signal integral calibration data. The top plot is the signal amplitude produced at a given pulse generator setting fitted to a straight line. The bottom plot shows the difference between the amplitudes measured and the amplitudes calculated from the fit parameters. The next three graphs follow the same format.

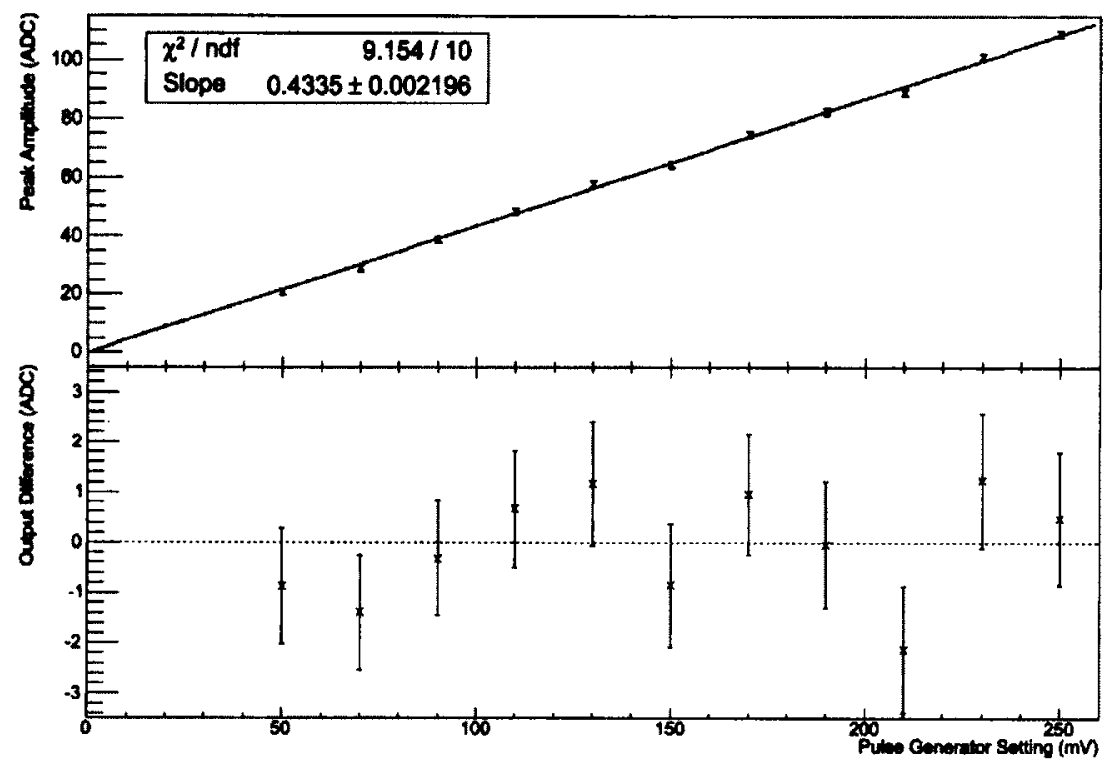

Figure 5.16: Peak amplitude calibration data. 


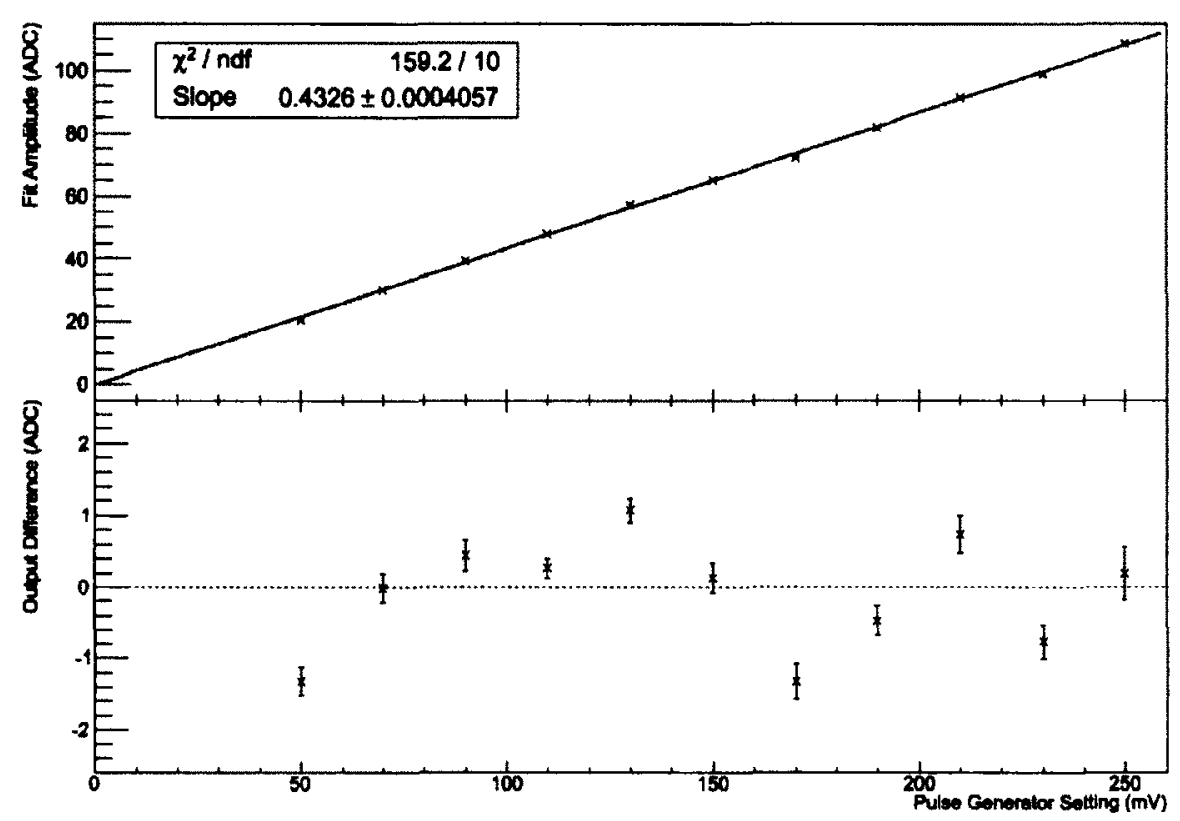

Figure 5.17: Fit amplitude calibration data.

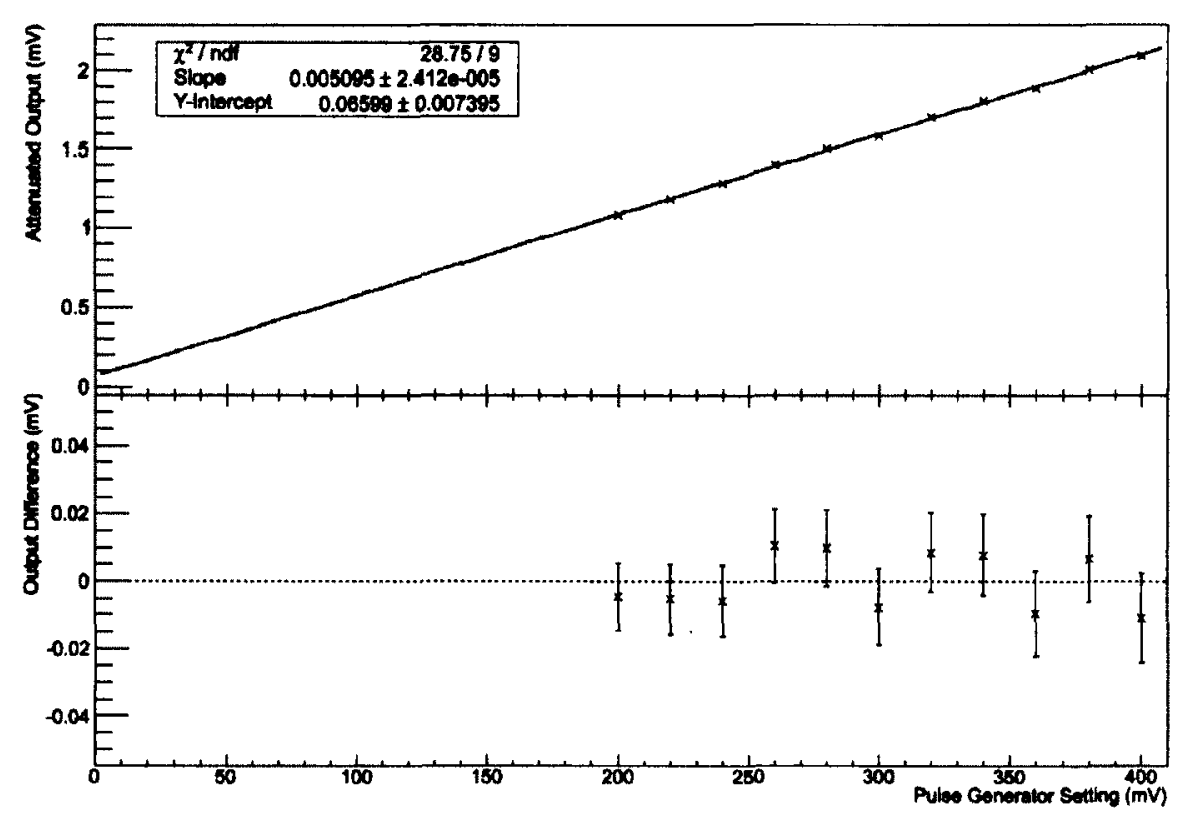

Figure 5.18: Output from pulse generator measured by the oscilloscope after attenuation versus the pulse generator voltage setting. Sample populations differ from linearity by $0.01688 \mathrm{mV}$, due to the minimum resolution of the oscilloscope. 
Combined, these fits give a calibration factor of:

$$
S_{A t t}=\frac{m_{A t t}}{m_{A}} S_{A}+b_{A t t}
$$

where $S_{A t t}, m_{A t t}$, and $b_{A t t}$ are the signal amplitude, slope, and y-intercept of the attenuated output of the pulse generator and $S_{A}$ and $m_{A}$ are the signal amplitude and slope of one of the three measurement methods. Knowing that the capacitor on the test pulse input to the preamplifier is $1 \mathrm{pF}$, the attenuated input voltage can be transformed into an amount of charge collected, $Q$, using:

$$
\begin{aligned}
Q & =f_{1} S_{A}+f_{2} k e / m V, \\
f_{1} & =6.24 \frac{m_{A t t}}{m_{A}} \\
f_{2} & =6.24 b_{A t t}
\end{aligned}
$$

where $k e$ is thousands of electrons collected. The uncertainty on this calibration factor is dominated by the test input's $1 \mathrm{pF}$ capacitor, which has an uncertainty of $25 \%$. Statistical uncertainty on the calibration plots contributes approximately $5 \%$, depending on the measurement method. The average charge collected for a single charged particle for each of the three measurement methods for data set 2 is given in Table 5.3. The measurement methods produce consistent results, although the signal integral is consistently greater than the other measures. This is possibly due to the signal tail present in the diamond waveforms that is not present in the calibration data. The data presented throughout the rest of this chapter are for the fit amplitude measurement, except where noted otherwise. 


\begin{tabular}{|l|l|}
\hline Measurement & Mean (ke) \\
\hline Peak Amplitude & $3.97 \pm 0.17$ \\
Fit Amplitude & $3.97 \pm 0.17$ \\
Signal Integral & $4.11 \pm 0.17$ \\
\hline
\end{tabular}

Table 5.3: Average signal amplitude and statistical uncertainty from three different measurement methods over the course of data set 2.

\subsubsection{Data Quality}

An investigation was made to assess the quality and consistency of the data. The first study was a plot of the signal amplitude as a function of scan number. The quick drop in amplitude during the first hour, shown in Figure 5.19, is consistent with the diamond detector being exposed to UV light and induced polarization effects due to changing the applied bias voltage, neither of which could be avoided during the installation process. Figure 5.20 shows a data set where the diamond was neither exposed to UV light or polarization effects, leading to a stable amplitude throughout the data set. Inspection of these figures allowed for selection of regions where the data was stable. Regions where the amplitude was varying, such as the initial regions, were removed.

The timing of the peak position of each scan was determined and shown to be quite stable, as shown in Figures 5.21-5.22. The variations seen are due to changes in the trigger logic between (or during) data sets.

Measurements of the pedestal value with time were taken for each data set. An example is given in Figure 5.23. These plots show a 24 hour periodic structure, likely caused by fluctuations in temperature in the vicinity of the electronics. The 


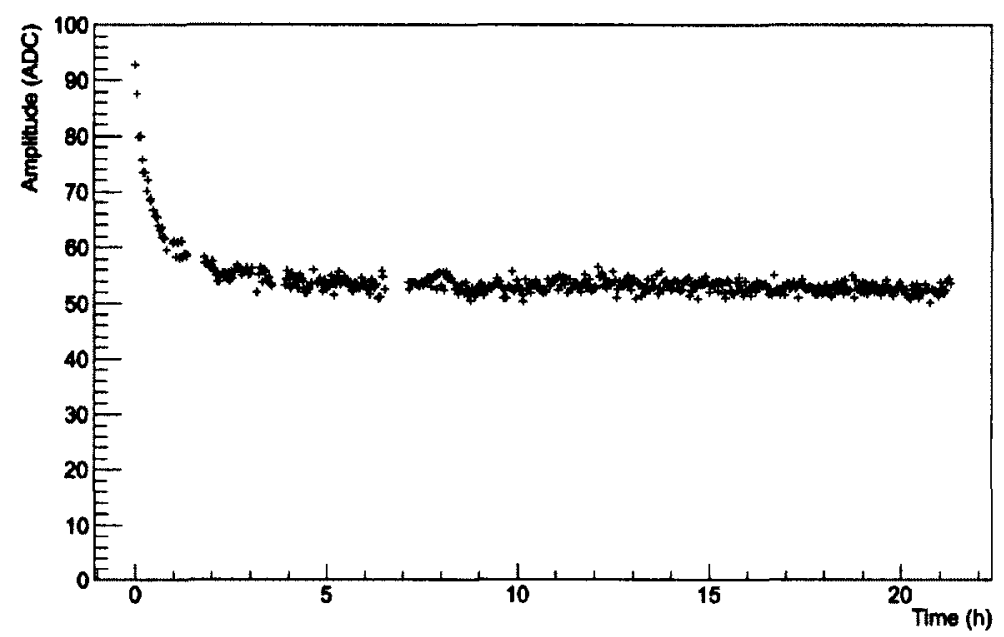

Figure 5.19: Measurement of average peak amplitude per scan for data set 1. Note the large initial decrease in amplitude.

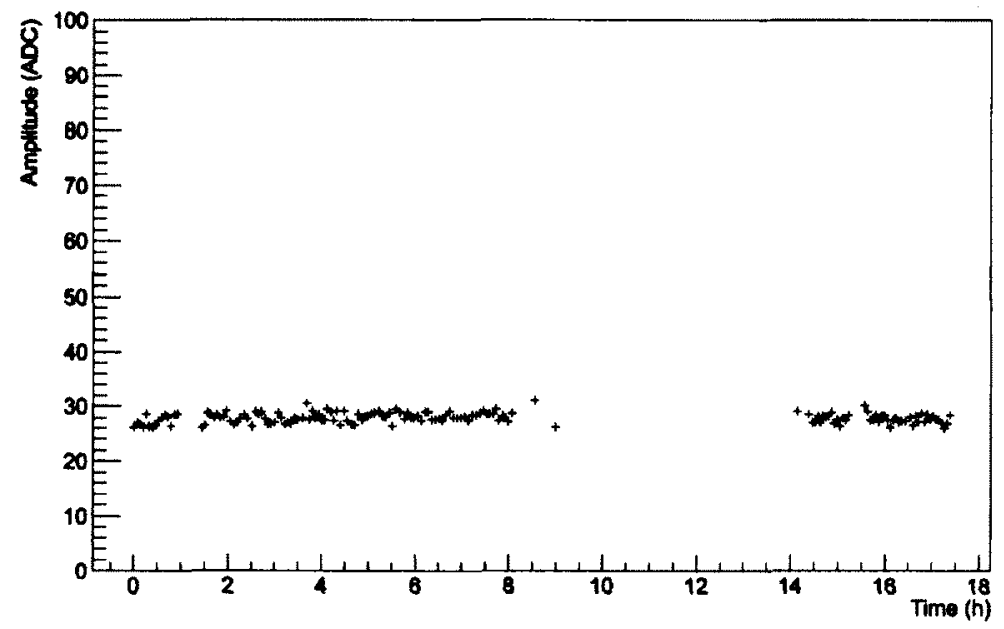

Figure 5.20: Measurement of average peak amplitude per scan for data set 5. The amplitude is stable throughout an 18 hour period, even though beam was lost for a period of nearly 5 hours. 


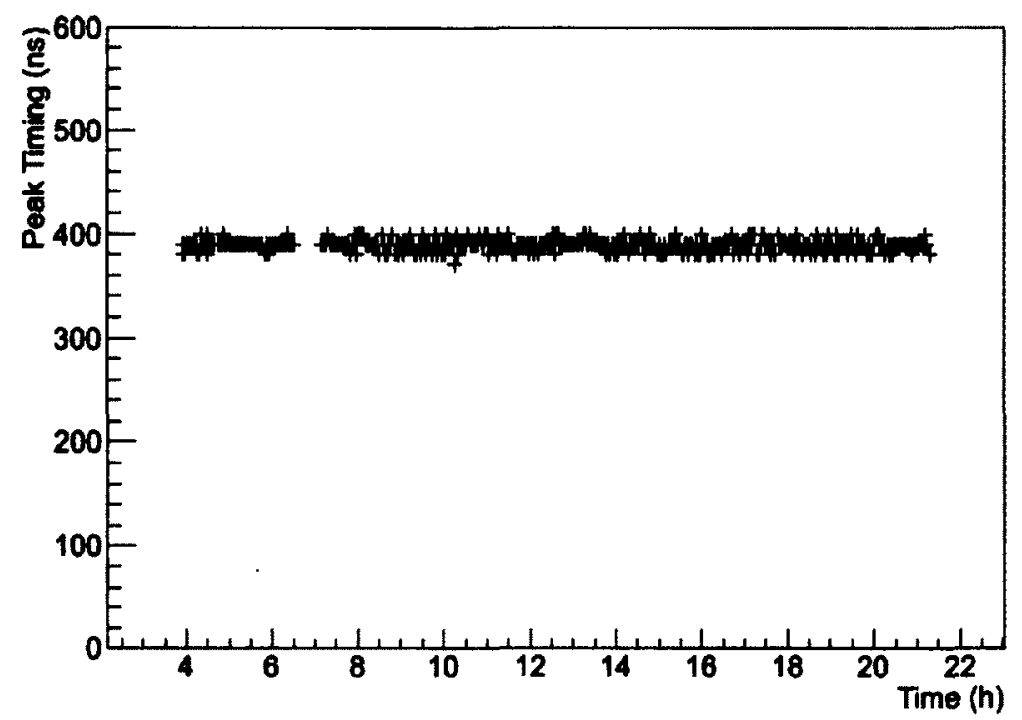

Figure 5.21: Variation in peak timing during data set 1. The peak position remains stable for over 20 hours. The band structure is due to the time resolution of the FADC system.

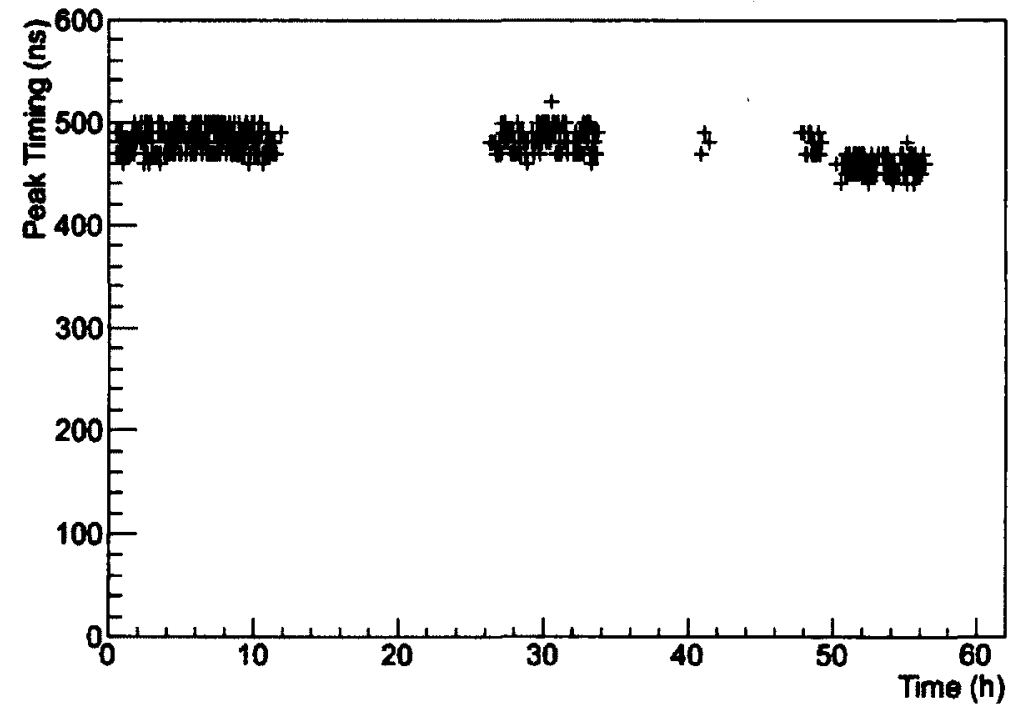

Figure 5.22: Variation in peak timing during data set 4. The peak position has shifted by $100 \mathrm{~ns}$ with respect to data set 1 due to changes in the trigger logic. The shift at 50 hours is due to changes in the trigger logic made in preparation for switching to a negative beam polarity. 


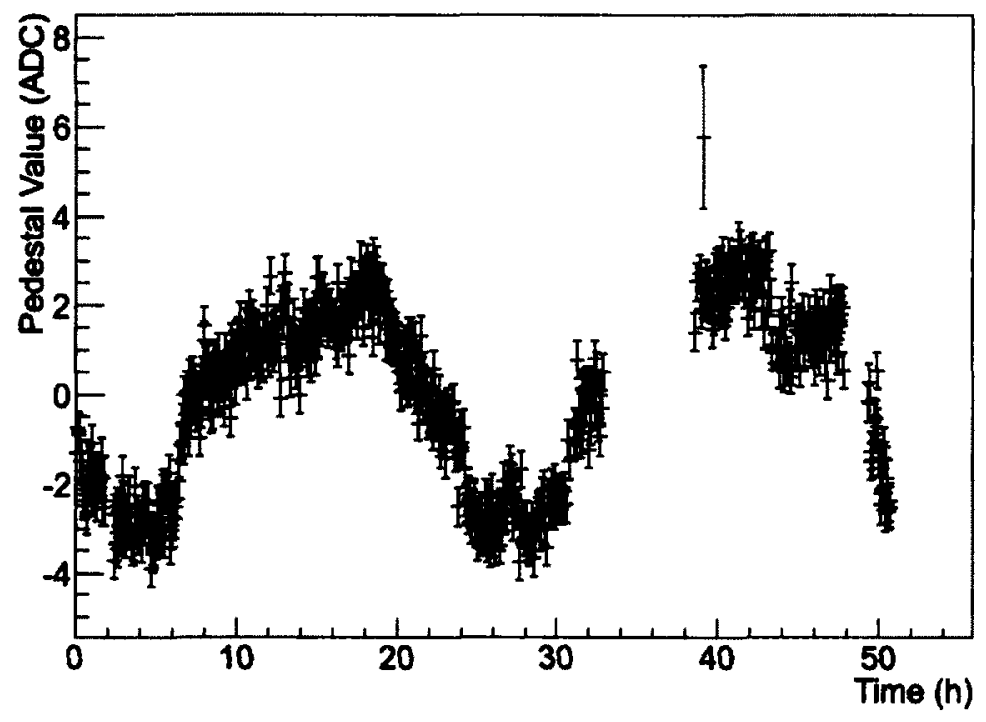

Figure 5.23: Variations in pedestal value in data set 2.

effective removal of the pedestal was thus necessary to compare data from different sets.

The signal response of the diamond was measured after each scan and was observed to vary after long periods without beam, as seen in Figure 5.24.

\subsection{Spatial Response}

The spatial response of the diamond was examined by matching a diamond signal output to a hit position in the USBpix for each event. By averaging the diamond detector pulses associated with each pixel and measuring the amplitude of the averaged pulse, we get an estimate of how the charge deposited by a single particle varied across the surface of the diamond detector. An example of this is given in Figure 5.25. This section will describe the analysis of the spatial response data, describe the unexpected differences between diamond detector bias polarities, and describe a 


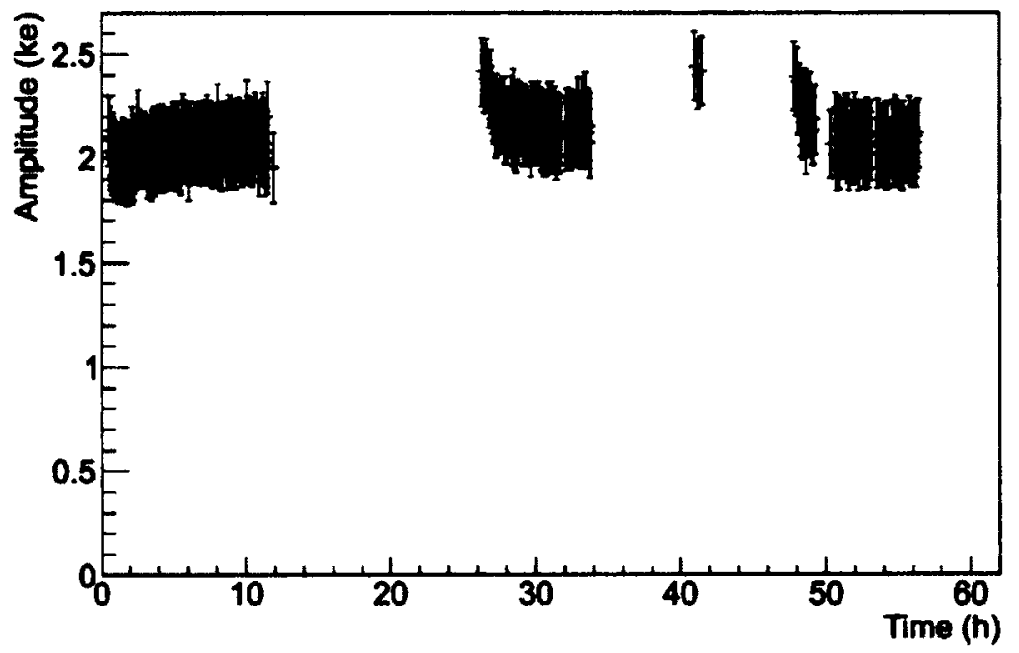

Figure 5.24: Variations in the signal amplitude measured per scan in data set 4. Note the increase in amplitude followed by a quick decay after periods without beam. These periods lasted for up to 14 hours.

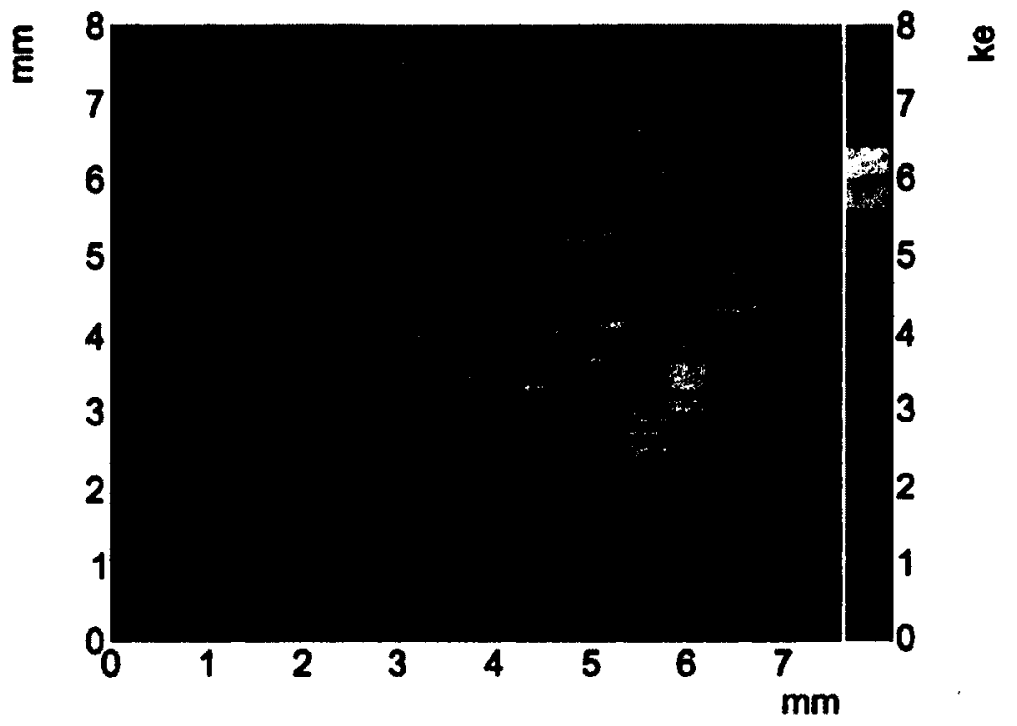

Figure 5.25: Averaged amplitude map for data set 2. Each $50 \mu \mathrm{m} \times 400 \mu \mathrm{m}$ pixel represents the average charge deposited by a single particle in said pixel. 


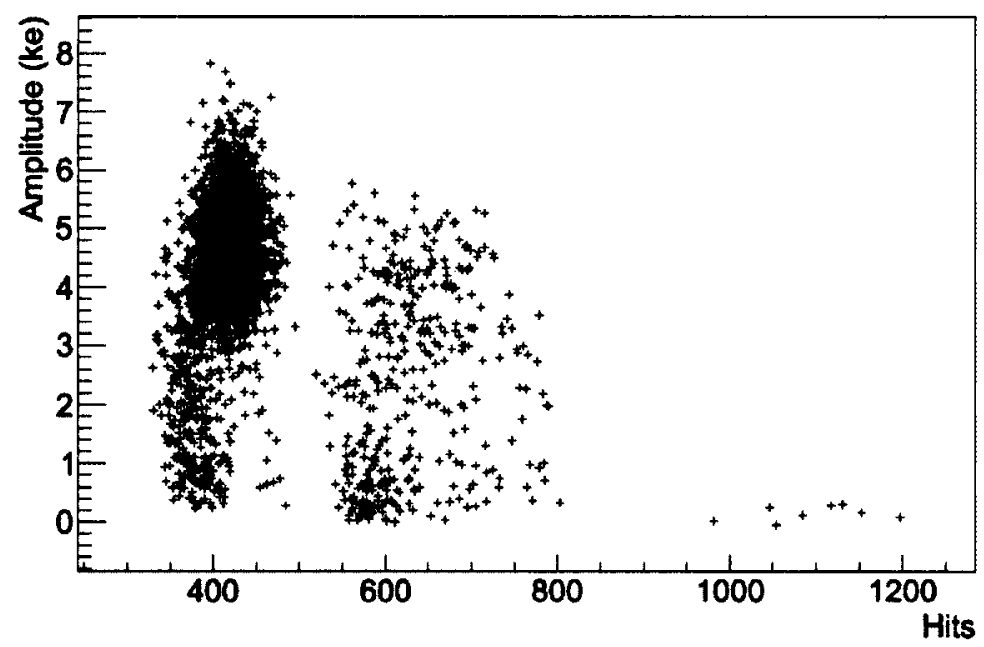

Figure 5.26: Comparison of diamond signal amplitude to number of hits in USBpix for each pixel.

method used to combine data sets to modify the pixel resolution.

A major source of uncertainty in this analysis is the effect of multiple scattering on the hit position registered by the USBpix. The distance between the USBpix and the diamond detector was kept to a minimum to reduce the effect of multiple scattering, as discussed in Chapter 4. Assuming a perpendicular incident particle, the deflection due to multiple scattering between the detectors resulted in, on average, only slightly more than $50 \%$ of particles being detected in the correct pixel. Since the angular distribution of the M11 beam is unknown this is likely an underestimate of the number of falsely identified hit positions. The falsely identified positions reduce the amplitude variations from pixel to pixel. Thus, the variation in signal response across the surface of a pCVD diamond detector is likely greater than reported here. It is possible that a Monte Carlo simulation could be used to determine the true signal response in each pixel. This was not attempted here due to time constraints.

A study was made to check that the amplitude variations abserved in the 
diamond detector were not simply due to an artifact from the pixel detector, related to the number of hits in each pixel. The diamond detector amplitude was plotted as a function of the number of hits in the pixel detector as shown in Figure 5.26. This figure shows that there is no correlation between signal amplitude and the number of pixel hits. Several different populations are observable, which represent the centre of the diamond detector (high amplitude, low number of hits), its edge region (low amplitude, low number of hits), and the edge pixels of the USBpix (varying amplitude, high number of hits). Refer to Figure 5.3 for a representation of the USBpix cell occupancy. Thus, the amplitude variations observed within the diamond are not due to the number of hits in each region.

\subsubsection{Signal Amplitude Variations with Bias Voltage Polarity}

All negatively biased data sets show similar high amplitude pixels (hot spots) in similar regions, once the rotation of the diamond has been accounted for. The same is true of the two positively biased data sets. Large differences are observed between amplitude maps of different bias polarities, as shown in Figure 5.27. Not only are the amplitudes different by a factor of 2 , but the hotspots appear in different positions, indicating that the same regions of the diamond behave differently depending on the bias voltage applied.

The spectra of the amplitude maps were also examined, with the expected result; no significant difference in results between data sets where the same bias polarity was applied are observed. Figure 5.28 shows examples of the spectra for both negative and positive applied bias data sets. Table 5.4 lists the mean and 


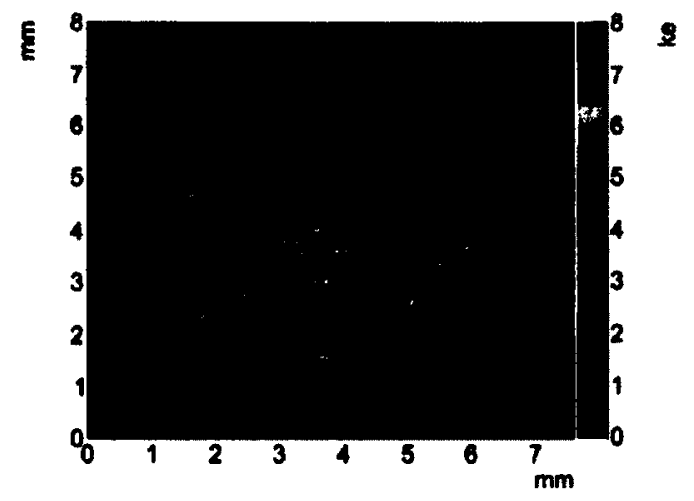

(a) Negative bias voltage

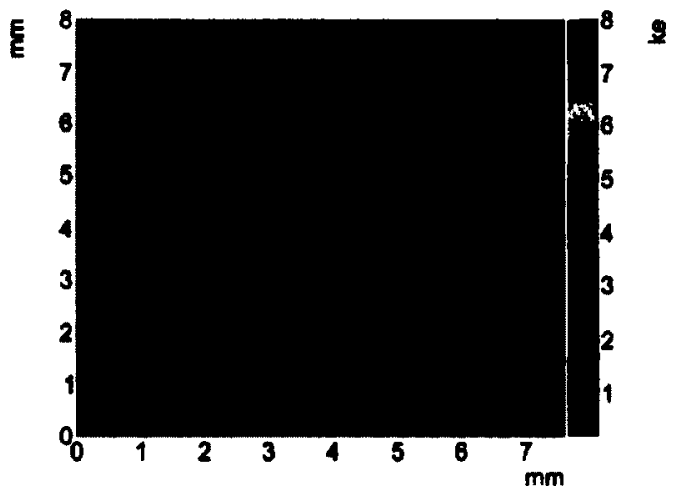

(b) Positive bias voltage

Figure 5.27: Diamond detector amplitude maps. The large variation between these plots is due to the change in bias voltage polarity.

standard deviation for all data sets.

\subsubsection{Combined Spatial Resolution Data}

Due to the design of the USBpix detector pixels, the resolution along one axis is much worse than the resolution of the other. The diamond detector was rotated $90^{\circ}$ and an attempt was made to combine the resulting data (from data sets 2 and 3 ) to ameliorate this problem. Two orientations of the diamond detector are insufficient to accomplish this analytically, so a numerical solution was attempted. To determine whether one combination of data was better than another, a goodness of fit variable was necessary. The sum of the $n$ highest amplitude pixels in the combined map, where $n$ is varied between 10 and 1000 , proved to be a useful measure. The two amplitude maps used to study the combination are shown in Figure 5.29.

To combine the data sets the pixels in both maps were broken down into $50 \mu \mathrm{m}$ x $50 \mu$ m pixels. Each 'sub' pixel was given the same amplitude, which was normalized 


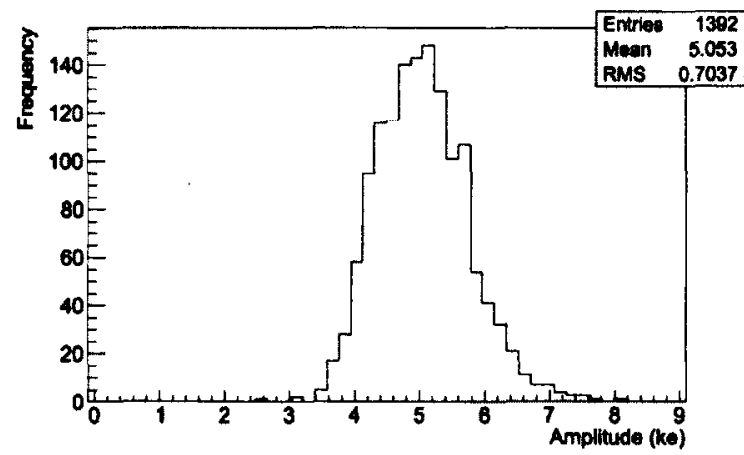

(a) Negative bias voltage

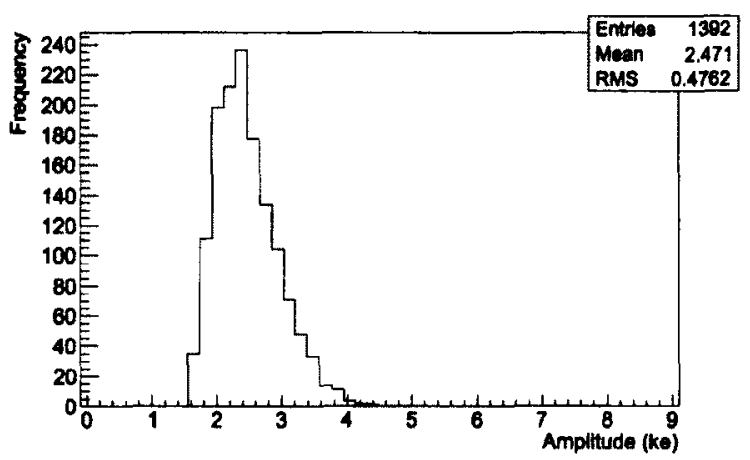

(b) Positive bias voltage

Figure 5.28: Amplitude spectra for data sets 3 and 4. To ensure that only the signal region of the diamond is reported in the spectra, a radial cut of $3 \mathrm{~mm}$ from the centre of the USBpix is applied. This ensures that the edge pixels are avoided by a safe margin.

\begin{tabular}{|c|c|c|c|c|}
\hline Data Set & Mean (ke) & $\sigma(\mathrm{ke})$ & Bias Polarity & Beam Polarity \\
\hline 1 & 4.73 & 0.70 & - & + \\
2 & 4.86 & 0.72 & - & + \\
3 & 5.05 & 0.70 & - & + \\
4 & 2.47 & 0.48 & + & + \\
5 & 2.84 & 0.51 & + & - \\
6 & 4.58 & 0.56 & - & - \\
7 & 4.81 & 0.69 & - & + \\
\hline
\end{tabular}

Table 5.4: Signal amplitude mean and standard deviation for a single particle passing through the diamond detector for all data sets. 

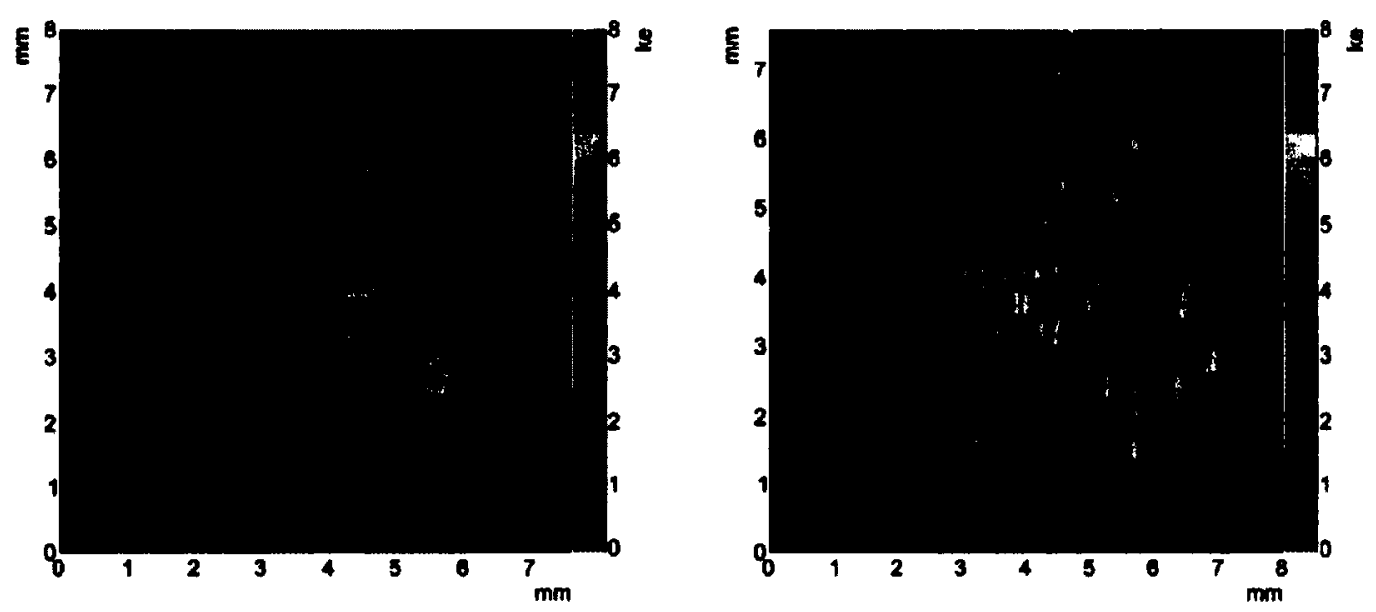

Figure 5.29: Amplitude maps from data sets 2 (left) and 3 (right). Some of the suspected hot spots have been outlined. Note that data set 3 was rotated back $90^{\circ}$ during the analysis for ease of comparison. 
in such a way that the summation of sub-pixels would result in the original value. The amplitude map of data set 2 was then moved over the surface of data set 3's amplitude map in $50 \mu \mathrm{m}$ increments. These increments are labeled as ishift and jshift for horizontal and vertical shifts respectively. The amplitudes of each sub-pixel were averaged to form a combined amplitude map. Pixels without a corresponding partner were ignored, giving a maximum number of pixels in the combined maps of $152 \times 152$.

As a quality of fit indicator, the $10(100,1000)$ combined pixels with the highest amplitude for each increment were summed and plotted in Figure 5.30 (5.31, 5.32). The sum of the 10 highest amplitude pixels proved to be an unstable measure, as its maximum occurred at an impossible shift value, while the 100 and 1000 pixel sum maps show the same maximum sum region. The maximum occurs at points $(4,-3)$ and $(4,-1)$ for 100 and 1000 pixel sum maps respectively. The 1000 pixel sum was chosen as the best quality of fit indicator, since its amplitude map shows the least amount of variation. In other words, the amplitude map for data set 2 is shifted 200 $\mu \mathrm{m}$ left and $50 \mu \mathrm{m}$ up with respect to the amplitude map for data set 3 .

The results of the combination of data at this location for $50 \times 50,100 \times 100$, and $200 \times 200 \mu \mathrm{m}^{2}$ pixels are shown in Figures $5.33-5.35$. The combination seems to have been a success; the hot spots are depicted in the expected locations. It is difficult to trust the validity of the $50 \times 50$ and $100 \times 100 \mu \mathrm{m}^{2}$ results, as our original pixels were only $50 \times 400 \mu \mathrm{m}^{2}$. However, the $200 \times 200 \mu \mathrm{m}^{2}$ pixel data is believed to be a reasonable indicator of the signal variations to be expected from a pCVD diamond detector.

Amplitude spectra for each pixel size, as well as data sets 2 and 3 , were created, as shown in Figures 5.36 - 5.40. Note that a radius cut was applied in all cases to isolate the centre of the diamond detector from its edge regions. The means and 


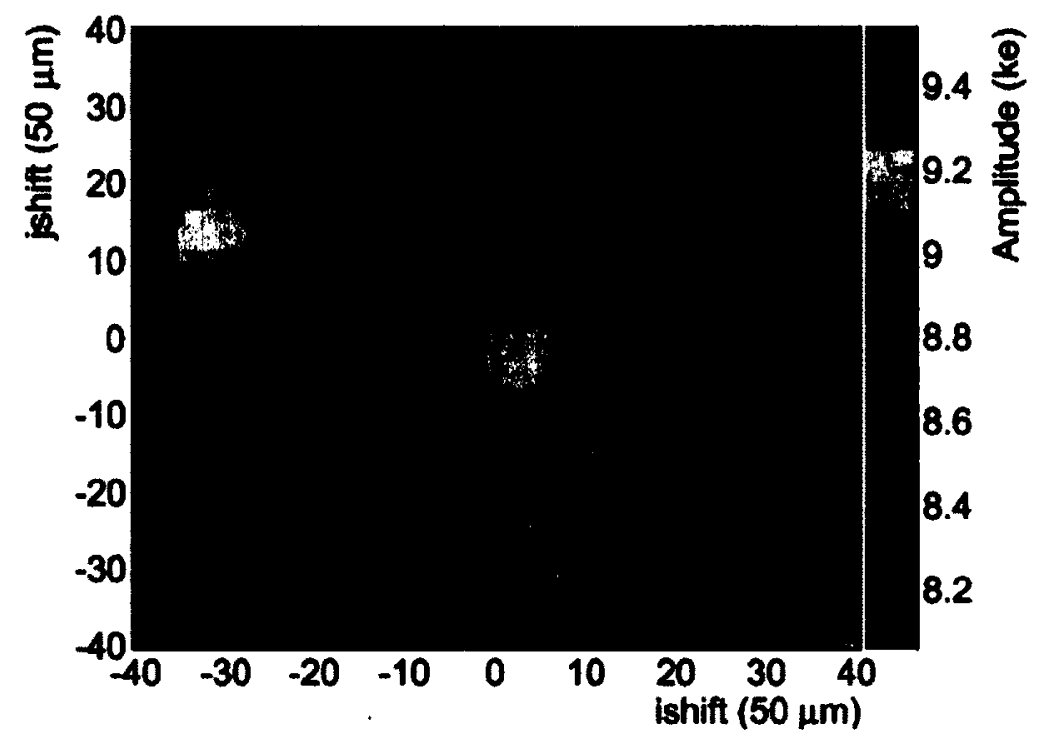

Figure 5.30: Map of the sum of the 10 highest amplitude combined pixels for each 50 micron shift. Note that the axes represent the opposite movement of data set 2's amplitude map; a positive ishift moves the map to the left, a positive jshift down.

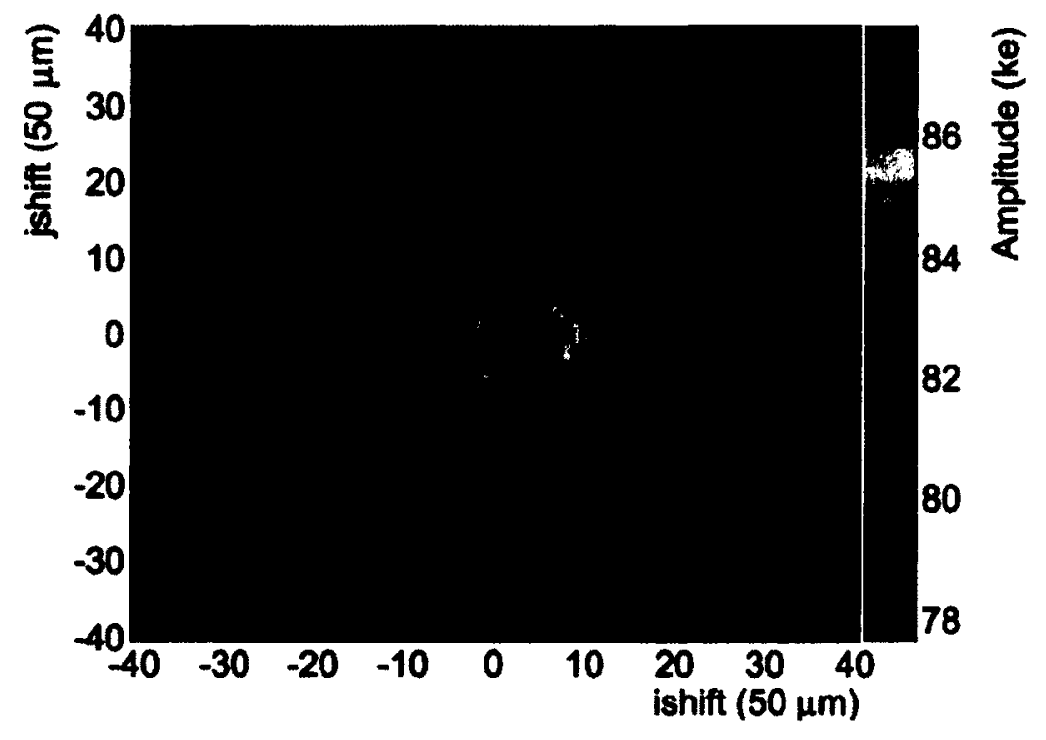

Figure 5.31: Map of the sum of the 100 highest amplitude combined pixels for each 50 micron shift. 


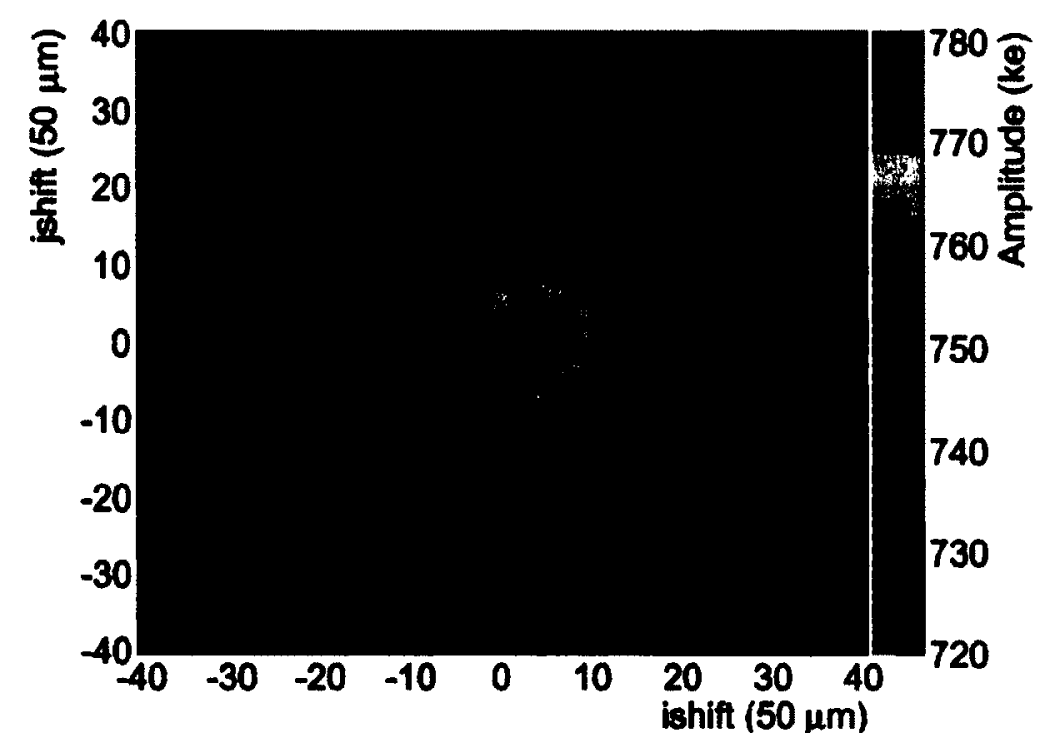

Figure 5.32: Map of the sum of the 1000 highest amplitude combined pixels for each 50 micron shift.

standard deviations of these distributions are listed in Table 5.5. The means and standard deviations were also normalized to the original pixel size of $50 \mu \mathrm{m} \times 400 \mu \mathrm{m}$. This was done to better represent the charge deposited by a single particle and to allow comparisons between data sets. The results are consistent with expectations; the normalized mean charge collected for all three combined amplitude maps is roughly the average of the two data sets used to form them and the normalized standard deviation improves for the combined amplitude data with larger pixel sizes. However, the variations seen in the normalized standard deviation of the combined amplitude data are lower than one would expect, likely due to the algorithm used to combine data sets 2 and 3 . As such, the data from data sets 2 and 3 are believed to better represent the signal amplitude variations of a pCVD diamond detector.

The signal amplitude distribution gathered from the data set 3 amplitude map will be used to improve simulations of the Mini-FCal response and, in particular, to 


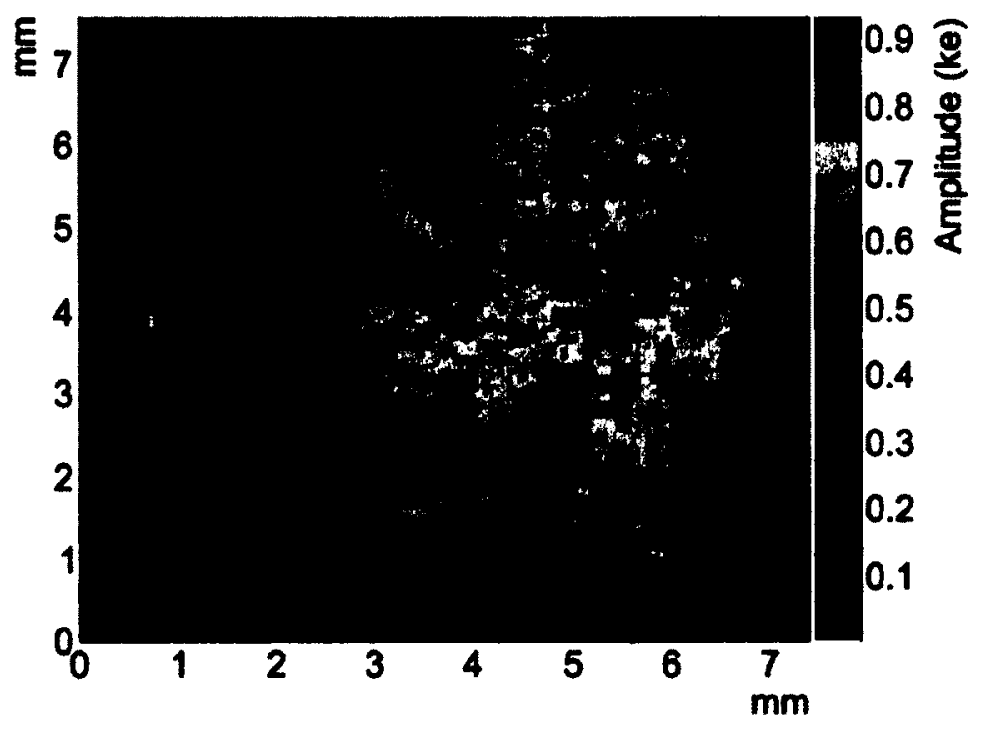

Figure 5.33: Combined amplitude map for data sets 2 and 3 in $50 \times 50 \mu \mathrm{m}^{2}$ pixels.

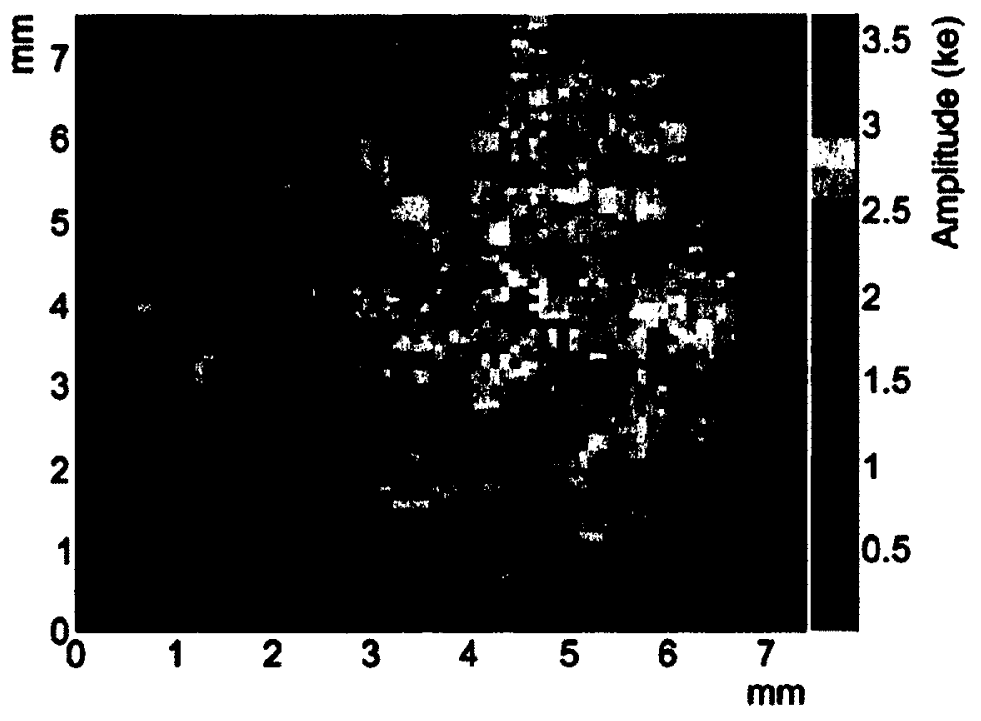

Figure 5.34: Combined amplitude map for data sets 2 and 3 in $100 \times 100 \mu m^{2}$ pixels. 


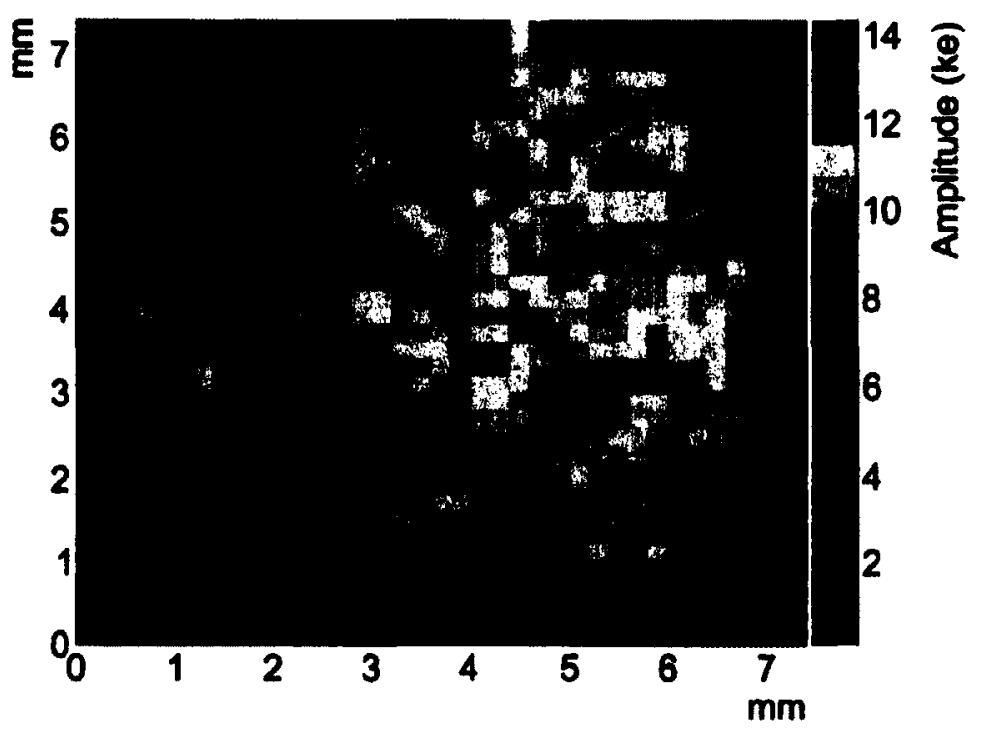

Figure 5.35: Combined amplitude map for data sets 2 and 3 in $200 \times 200 \mu m^{2}$ pixels.

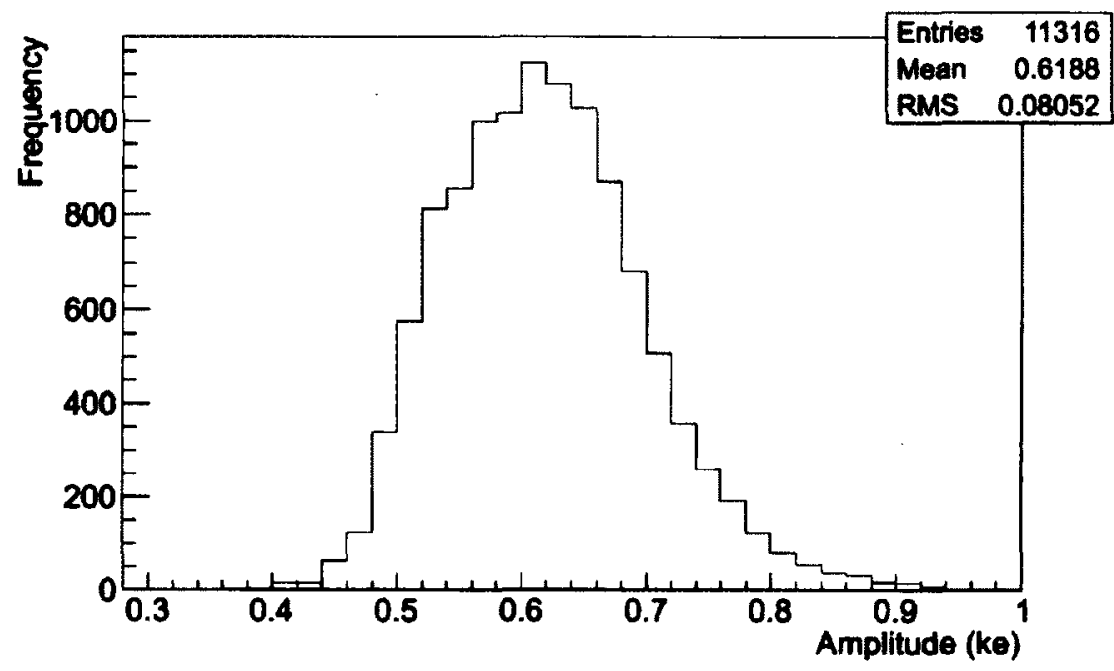

Figure 5.36: Amplitude spectrum for 50 micron $\times 50$ micron pixels. 


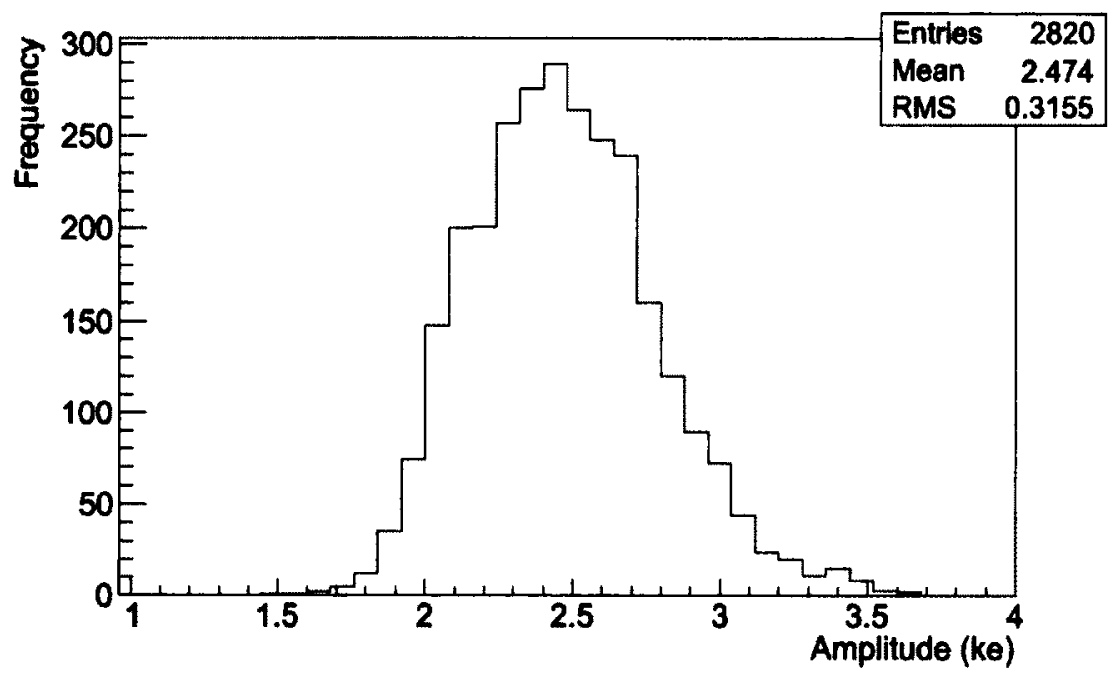

Figure 5.37: Amplitude spectrum for 100 micron $\times 100$ micron pixels.

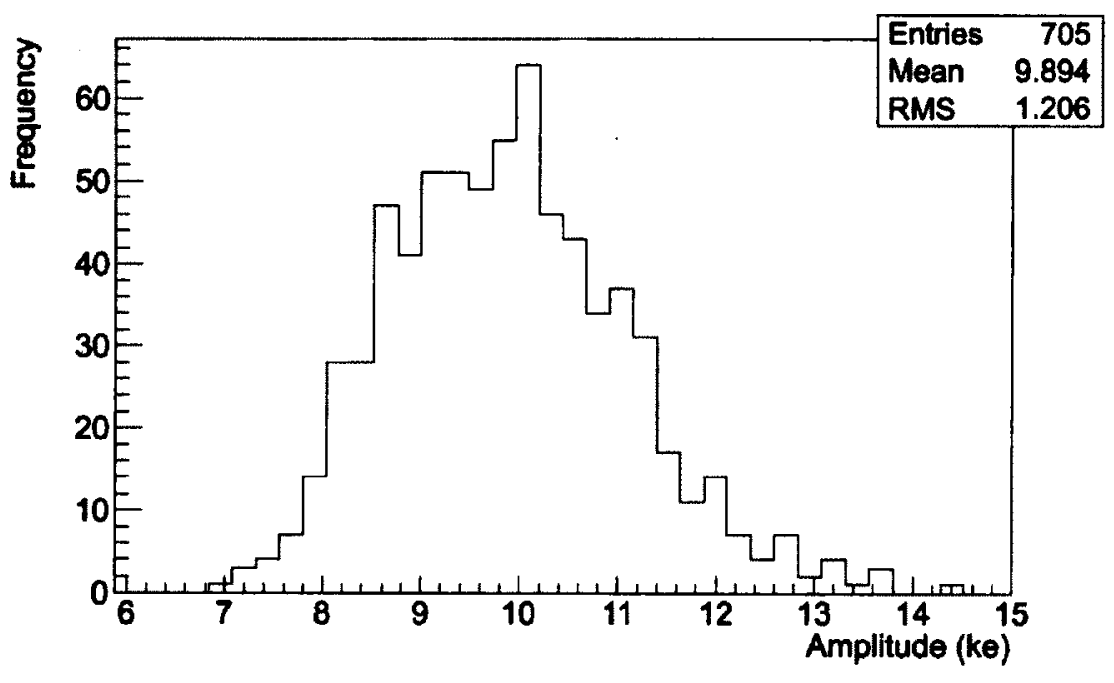

Figure 5.38: Amplitude spectrum for 200 micron $\times 200$ micron pixels. 


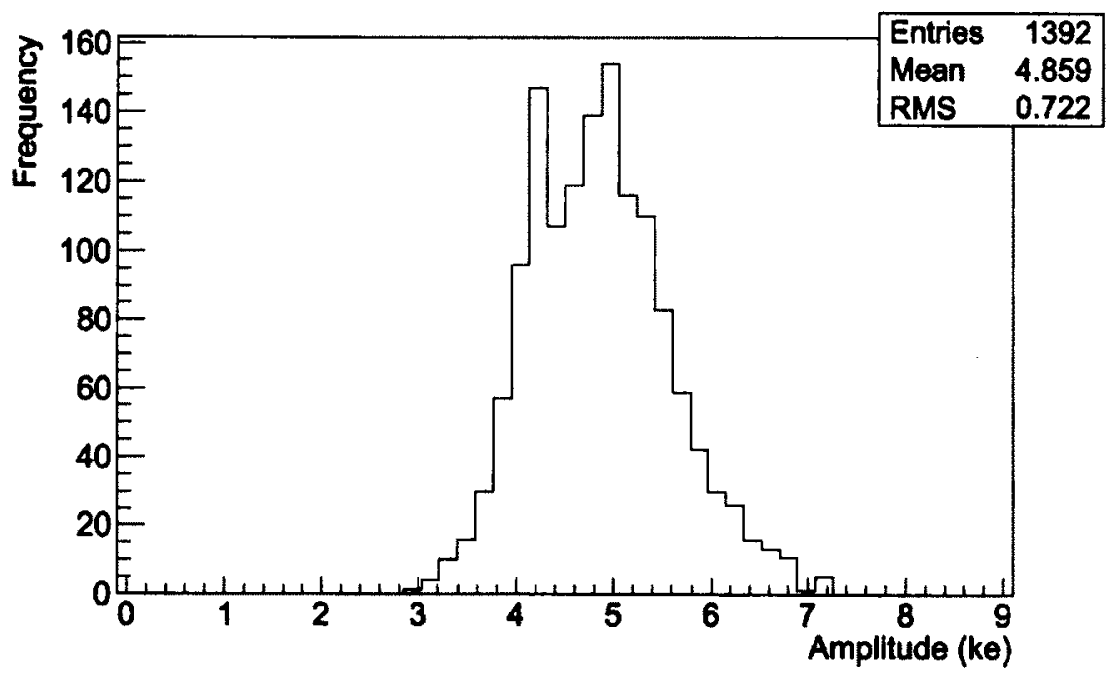

Figure 5.39: Amplitude spectrum for data set 2.

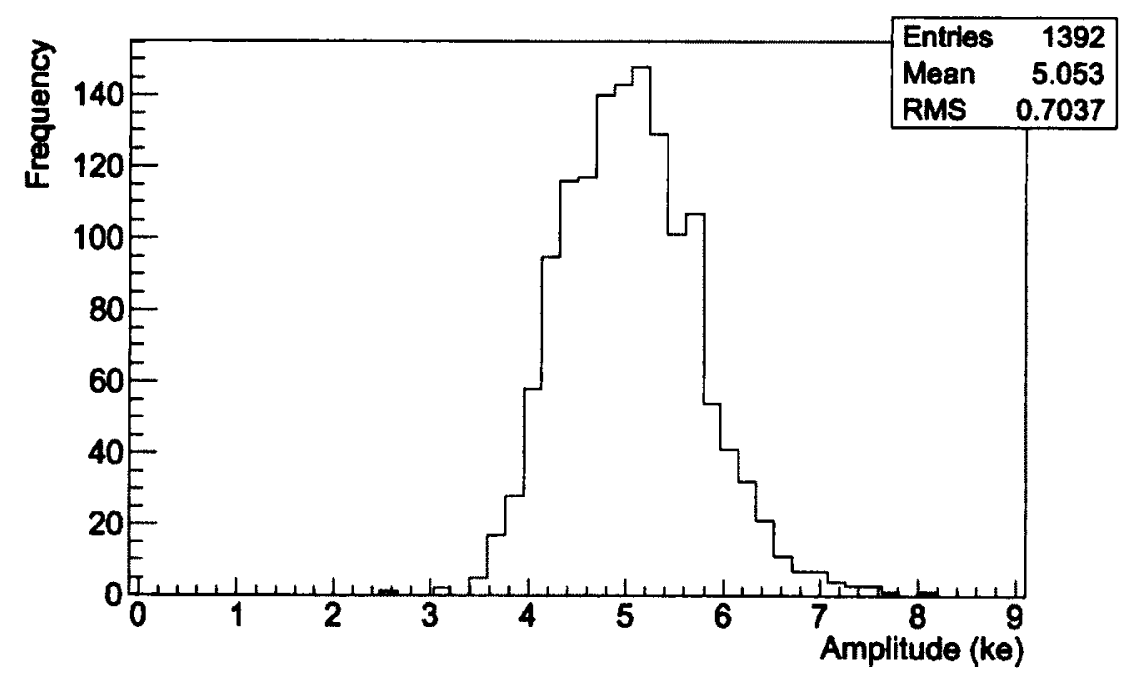

Figure 5.40: Amplitude spectrum for data set 3. 


\begin{tabular}{|c|c|c|c|c|}
\hline Pixel Size & Mean & $\sigma$ & $\begin{array}{c}\text { Normalized } \\
\text { Mean (ke) }\end{array}$ & $\begin{array}{c}\text { Normalized } \\
\sigma(\mathrm{ke})\end{array}$ \\
\hline $50 \times 50 \mu \mathrm{m}^{2}$ & $0.62\left(\mathrm{ke}^{*} \mu \mathrm{m}^{2}\right)$ & $0.08\left(\mathrm{ke}^{*} \mu \mathrm{m}^{2}\right)$ & 4.95 & 0.64 \\
\hline $100 \times 100 \mu \mathrm{m}^{2}$ & $2.5\left(\mathrm{ke}^{*} \mu \mathrm{m}^{2}\right)$ & $0.3\left(\mathrm{ke}^{*} \mu \mathrm{m}^{2}\right)$ & 4.95 & 0.63 \\
\hline $200 \times 200 \mu \mathrm{m}^{2}$ & $9.9\left(\mathrm{ke}^{*} \mu \mathrm{m}^{2}\right)$ & $1.2\left(\mathrm{ke}^{*} \mu \mathrm{m}^{2}\right)$ & 4.95 & 0.60 \\
\hline $\begin{array}{c}50 \times 400 \mu \mathrm{m}^{2} \\
(\text { data set } 2)\end{array}$ & $4.86(\mathrm{ke})$ & $0.72(\mathrm{ke})$ & - & - \\
\hline $\begin{array}{c}50 \times 400 \mu \mathrm{m}^{2} \\
(\text { data set } 3)\end{array}$ & $5.05(\mathrm{ke})$ & $0.70(\mathrm{ke})$ & - & - \\
\hline
\end{tabular}

Table 5.5: Mean and standard deviation of the three combined amplitude maps and the data sets used to create them. Normalizing these results to the original pixel size produces the normalized mean and standard deviation reported here, which represent the charge deposited by a single particle in the diamond detector.

judge the effect these amplitude variations have on the energy resolution of the MiniFCal. If the variations prove overly detrimental then additional tests will be required to confirm, and improve upon, the results presented here. In particular, the use of a beam telescope is recommended, to allow for detailed track reconstruction and improved hit position identification. 


\section{Chapter 6}

\section{Summary and Outlook}

The HL-LHC will require detectors to endure radiation levels a full order of magnitude greater than those present at the LHC. R\&D is ongoing to develop the detector technology and techniques necessary to survive in this environment in preparation for the scheduled upgrades. Many detector systems rely on semiconducting materials, several of which are under investigation, including diamond detectors. The high cost of diamond detectors has so far prevented their widespread application. The MiniFCal detector, a detector proposed for the HL-LHC, would use diamond detectors, if they prove suitable and can be obtained in large quantities at an acceptable price.

The irradiation test at TRIUMF in September of 2010 exposed four diamond detectors to an integrated particle flux greater than the flux expected within the Mini-FCal throughout the lifetime of the HL-LHC. All four detectors remained operational, although at $5 \%$ of their original signal amplitude. Diamond detectors are thus radiation hard enough to survive the conditions expected within the Mini-FCal, but require energy corrections that vary with the integrated particle flux within the detector. With 8000 diamond detectors in each Mini-FCal all exposed to varying 
amounts of particle flux, these energy corrections are expected to be quite complex.

The spatial uniformity test at TRIUMF in September of 2011 exposed a single diamond detector to a particle beam and measured the detector's response with respect to the particle's position in a secondary detector (the USBpix detector). The variations observed in signal amplitude throughout the bulk of the diamond detector proved more irregular than expected, with numerous confirmed hot spots. The diamond detector's response to varying bias polarities was equally unexpected. Not only was there a factor of two difference between the signal amplitude between polarities, but the hot spots were observed in different areas, implying that the internal structure of pCVD diamond differs drastically across the bulk of the material in as little as $0.7 \mathrm{~mm}$. Given that pCVD diamond grows from a multitude of tiny crystals on the substrate side to large crystals on the growth side, it is not too surprising that the properties vary across the bulk of the diamond. The response of the diamond detector was also observed to increase after long periods without beam (which will occur regularly at the HL-LHC) before decaying back to the average signal amplitude.

Two orientations of the diamond detector were examined during the spatial uniformity test. The alignment of the two orientations was obtained by varying the overlapping surface area between the two data sets and measuring the sum of the 1000 highest amplitude pixels. The alignment with the highest sum was considered to be the best fit. This proved to be quite successful, and was used to produce combined signal amplitude maps for varying pixel sizes. The combined pixel sizes are difficult to validate; the normalized standard deviations of their signal amplitude spectra differ less than would be expected from such large changes in pixel area. This is likely due to the algorithm used to produce the combined pixel maps, which simply divided the original pixels into eight pixels of equal amplitude, smoothing out the 
variations. To improve the results for the combined pixel maps, an algorithm that produces a continuous amplitude distribution when broken down into smaller pixel sizes is suggested.

The scattering of incident particles between the two detectors led to an estimated $50 \%$ of the particle positions being falsely identified. This smooths out the variations in signal amplitude between pixels. As such, the variations in signal amplitude in pCVD diamond detectors are believed to be larger than those observed here. To improve the results, a Monte Carlo simulation could be used to determine the true signal amplitude in each pixel.

The amplitude spectrum from data set 3 will be used in the simulation of the response of the Mini-FCal at the HL-LHC. If the variations in signal amplitude within pCVD diamond detectors prove overly detrimental to the Mini-FCal's performance, the measurements presented here will have to be confirmed and a better estimate of the variation gathered. A new test would require a beam telescope that provided a track resolution at the test detector of better than $50 \mu \mathrm{m}$ for both axes. This would vastly improve the number of correctly identified hit positions.

The variations in response observed in pCVD diamond detectors in high particle flux environments, the large variations in signal amplitude throughout the bulk, the observation of notable hot spots, the variations in response due to different bias polarities, and the variations in response after long periods without beam all indicate that $\mathrm{pCVD}$ diamond detectors require further $\mathrm{R} \& \mathrm{D}$ if they are to be used in calorimeters that sit in a very high particle flux environment. 


\section{References}

[1] K. Nakamura and The Particle Data Group, "Review of particle physics," Journal of Physics G: Nuclear and Particle Physics, vol. 37, no. 7A, p. 075021, 2010.

[2] P. W. Anderson, "Plasmons, gauge invariance, and mass," Phys. Rev., vol. 130, pp. 439-442, Apr 1963.

[3] P. W. Higgs, "Broken symmetries and the masses of gauge bosons," Phys. Rev. Lett., vol. 13, pp. 508-509, Oct 1964.

[4] F. Englert and R. Brout, "Broken symmetry and the mass of gauge vector mesons," Phys. Rev. Lett., vol. 13, pp. 321-323, Aug 1964.

[5] G. S. Guralnik, C. R. Hagen, and T. W. B. Kibble, "Global conservation laws and massless particles," Phys. Rev. Lett., vol. 13, pp. 585-587, Nov 1964.

[6] S. Chatrchyan et al., "The CMS experiment at the CERN LHC," Journal of Instrumentation, vol. 3, no. 08, p. S08004, 2008.

[7] G. Aad et al., "The ATLAS experiment at the CERN Large Hadron Collider," Journal of Instrumentation, vol. 3, no. 08, p. S08003, 2008.

[8] A. Augusto Alves Jr et al., "The LHCb detector at the LHC," Journal of Instrumentation, vol. 3, no. 08, p. S08005, 2008. 
[9] K. Aamodt et al., "The ALICE experiment at the CERN LHC," Journal of Instrumentation, vol. 3, no. 08, p. S08002, 2008.

[10] G. Aad et al., "Observation of a centrality-dependent dijet asymmetry in leadlead collisions at $\sqrt{s_{\mathrm{NN}}}=2.76 \mathrm{TeV}$ with the ATLAS detector at the LHC," Phys. Rev. Lett., vol. 105, p. 252303, Dec 2010.

[11] L. Rossi, "Superconductivity: its role, its success and its setbacks in the Large Hadron Collider of CERN," Superconductor Science and Technology, vol. 23, no. 3 , p. $034001,2010$.

[12] L. Evans and P. Bryant, "LHC machine," Journal of Instrumentation, vol. 3, no. 08 , p. $S 08001,2008$.

[13] ATLAS Collaboration, "ATLAS Inner Detector: technical design report. 1." http://cdsweb. cern. ch/record/331063/. Accessed March 20, 2012.

[14] ATLAS Collaboration, "ATLAS Inner Detector: technical design report. 2." http://cdsweb. cern.ch/record/331064/. Accessed March 20, 2012.

[15] Sandro and Palestini, "The Muon Spectrometer of the ATLAS experiment," Nuclear Physics B - Proceedings Supplements, vol. 125, no. 0, pp. 337 - 345, 2003.

[16] A. Artamonov et al., "The ATLAS Forward Calorimeter," Journal of Instrumentation, vol. 3, no. 02, p. P02010, 2008.

[17] A. Glatte et al., "Liquid argon calorimeter performance at high rates," Nuclear Instruments and Methods in Physics Research Section A: Accelerators, Spectrometers, Detectors and Associated Equipment, vol. 669, no. 0, pp. 47-65, 2012. 
[18] P. Grenier et al., "Test beam results of 3D silicon pixel sensors for the ATLAS upgrade," Nuclear Instruments and Methods in Physics Research Section A: Accelerators, Spectrometers, Detectors and Associated Equipment, vol. 638, no. 1, pp. $33-40,2011$.

[19] C. D. Vi et al., "Radiation hardness properties of full-3D active edge silicon sensors," Nuclear Instruments and Methods in Physics Research Section A: Accelerators, Spectrometers, Detectors and Associated Equipment, vol. 587, no. 23, pp. $243-249,2008$.

[20] F. Nava, G. Bertuccio, A. Cavallini, and E. Vittone, "Silicon carbide and its use as a radiation detector material," Measurement Science and Technology, vol. 19, no. 10, p. 102001, 2008.

[21] V. Cindro et al., "The ATLAS beam conditions monitor," Journal of Instrumentation, vol. 3, no. 02, p. P02004, 2008.

[22] A. Bell et al., "Fast beam conditions monitor BCM1F for the CMS experiment," Nuclear Instruments and Methods in Physics Research Section A: Accelerators, Spectrometers, Detectors and Associated Equipment, vol. 614, no. 3, pp. 433 $438,2010$.

[23] R. S. Wallny, "Status of diamond detectors and their high energy physics application," Nuclear Instruments and Methods in Physics Research Section A: Accelerators, Spectrometers, Detectors and Associated Equipment, vol. 582, no. 3, pp. $824-828,2007$.

[24] J. Tseng, "ATLAS upgrade plans for the sLHC," Nuclear Physics B - Proceedings Supplements, vol. 177178 , no. 0, pp. $212-216,2008$. 
[25] C. Grah et al., "Polycrystalline CVD diamonds for the beam calorimeter of the ILC," Nuclear Science, IEEE Transactions on, vol. 56, pp. 462 -467, april 2009.

[26] P. Krieger, "The ATLAS liquid argon calorimeter: One year of LHC operation and future upgrade plans for HL-LHC," in Advancements in Nuclear Instrumentation Measurement Methods and their Applications (ANIMMA), 2011 2nd International Conference on, pp. 1-8, june 2011.

[27] H. Anderson and J. Ziegler, Hydrogen: Stopping Powers and Ranges in All Elements. Vol. 3 of The Stopping and Ranges of Ions in Matter. New York: Pergamon Press, 1977.

[28] A. B. Migdal, "Bremsstrahlung and pair production in condensed media at high energies," Phys. Rev., vol. 103, pp. 1811-1820, Sep 1956.

[29] U. Fano, "Penetration of protons, alpha particles, and mesons," Annual Review of Nuclear Science, vol. 13, no. 1, pp. 1-66, 1963.

[30] R. M. Sternheimer and R. F. Peierls, "General expression for the density effect for the ionization loss of charged particles," Phys. Rev. B, vol. 3, pp. 3681-3692, Jun 1971.

[31] R. M. Sternheimer, S. M. Seltzer, and M. J. Berger, "Density effect for the ionization loss of charged particles in various substances," Phys. Rev. B, vol. 26, pp. 6067-6076, Dec 1982.

[32] J. Lindhard and M. Scharff, "Energy dissipation by ions in the keV region," Phys. Rev., vol. 124, pp. 128-130, Oct 1961.

[33] W. R. Nelson, T. M. Jenkins, R. C. McCall, and J. K. Cobb, "Electron-induced 
cascade showers in copper and lead at $1 \mathrm{GeV}$," Phys. Rev., vol. 149, pp. 201-208, Sep 1966.

[34] G. Bathow, E. Freytag, M. Kbberling, K. Tesch, and R. Kajikawa, "Measurements of the longitudinal and lateral development of electromagnetic cascades in lead, copper and aluminum at $6 \mathrm{GeV}$," Nuclear Physics B, vol. 20, no. 3, pp. 592 $-602,1970$.

[35] W. Shockley, "Currents to Conductors Induced by a Moving Point Charge," Journal of Applied Physics, vol. 9, no. 10, pp. 635-636, 1938.

[36] S. Ramo, "Currents induced by Electron Motion," Proceedings of the IRE, vol. 27, p. 584, 1939.

[37] C. K. Jen, "On the Induced Current and Energy Balance in Electronics," Proceedings of the IRE, vol. 29, pp. 345-349, June 1941.

[38] G. Cavalleri, E. Gatti, G. Fabri, and V. Svelto, "Extension of Ramo's theorem as applied to induced charge in semiconductor detectors," Nuclear Instruments and Methods, vol. 92, pp. 137-140, Mar. 1971.

[39] Zhong and He, "Review of the Shockley-Ramo theorem and its application in semiconductor gamma-ray detectors," Nuclear Instruments and Methods in Physics Research Section A: Accelerators, Spectrometers, Detectors and Associated Equipment, vol. 463, no. 12, pp. 250 - 267, 2001.

[40] L. Rossi, P. Fischer, T. Rohe, and N. Wermes, Pixel Detectors: From Fundamentals to Applications (Particle Acceleration and Detection). New York: Springer, 2006. 
[41] U. Soldevila, "Radiation-hard silicon for HL-LHC trackers," Journal of Instrumentation, vol. 6, no. 12, p. C12035, 2011.

[42] M. Kohler et al., "Beam test measurements with planar and 3D silicon strip detectors irradiated to sLHC fluences," Nuclear Science, IEEE Transactions on, vol. 58, pp. $1308-1314$, June 2011.

[43] S. Parker, C. Kenney, and J. Segal, "3D - a proposed new architecture for solidstate radiation detectors," Nuclear Instruments and Methods in Physics Research Section A: Accelerators, Spectrometers, Detectors and Associated Equipment, vol. 395, no. 3 , pp. $328-343,1997$.

[44] J. Hiscock and A. T. Collins, "Comparison of diamond and silicon ultraviolet photodetectors," Diamond and Related Materials, vol. 8, no. 89, pp. 1753 - 1758, 1999.

"[45] M. Werner and R. Locher, "Growth and application of undoped and doped diamond films," Reports on Progress in Physics, vol. 61, no. 12, p. 1665, 1998.

[46] SpringerImages, "Schematic view of the pCVD diamond detector." http://www . springerimages .com/Images/RSS/1-10.1007_978-3-642-14142-3_7-45/. Accessed May 7, 2012.

[47] J. Isberg, J. Hammersberg, D. Twitchen, and A. Whitehead, "Single crystal diamond for electronic applications," Diamond and Related Materials, vol. 13, no. 2 , pp. $320-324,2004$.

[48] E. S. Technologies, "E6 CVD diamond eShop." http://www.e6cvd.com/. Accessed March 20, 2012. 
[49] J. B. Gibson, A. N. Goland, M. Milgram, and G. H. Vineyard, "Dynamics of radiation damage," Phys. Rev., vol. 120, pp. 1229-1253, Nov 1960.

[50] A. Vasilescu and G. Lindstrom, "Displacement damage in silicon." http:// sesam.desy.de/members/gunnar/Si-dfuncs.html. Accessed March 20, 2012.

[51] D. Asner et al., "Diamond pixel modules," Nuclear Instruments and Methods in Physics Research Section A: Accelerators, Spectrometers, Detectors and Associated Equipment, vol. 636, no. 1, Supplement, pp. S125 - S129, 2011.

[52] Gunnar and Lindstrm, "Radiation damage in silicon detectors," Nuclear Instruments and Methods in Physics Research Section A: Accelerators, Spectrometers, Detectors and Associated Equipment, vol. 512, no. 12, pp. 30-43, 2003.

[53] M. Bruinsma et al., "CVD diamonds in the BaBar radiation monitoring system," Nuclear Physics B - Proceedings Supplements, vol. 150, no. 0, pp. 164-167, 2006.

[54] D. Axen et al., "Diamond detector irradiation tests at TRIUMF," Journal of Instrumentation, vol. 6, no. 05, p. P05011, 2011.

[55] Cremat Inc., "Detection electronics by Cremat, Inc.." www.cremat.com. Accessed March 20, 2012.

[56] I. Peri, L. Blanquart, G. Comes, P. Denes, K. Einsweiler, P. Fischer, E. Mandelli, and G. Meddeler, "The FE-I3 readout chip for the ATLAS pixel detector," Nuclear Instruments and Methods in Physics Research Section A: Accelerators, Spectrometers, Detectors and Associated Equipment, vol. 565, no. 1, pp. 178 $187,2006$.

[57] Bonn University, "USBpix wiki." http://icwiki.physik.uni-bonn.de/ twiki/bin/view/Systems/UsbPix. Accessed March 20, 2012. 
[58] ROOT Development Team, "ROOT data analysis system." http://root.cern. ch/drupal/. Accessed March 25, 2012. 